\title{
ON THE VARIETY OF LAGRANGIAN SUBALGEBRAS, II
}

\author{
BY SAM EVENS AND JIANG-HUA LU
}

ABSTRACT. - Motivated by Drinfeld's theorem on Poisson homogeneous spaces, we study the variety $\mathcal{L}$ of Lagrangian subalgebras of $\mathfrak{g} \oplus \mathfrak{g}$ for a complex semi-simple Lie algebra $\mathfrak{g}$. Let $G$ be the adjoint group of $\mathfrak{g}$. We show that the $(G \times G)$-orbit closures in $\mathcal{L}$ are smooth spherical varieties. We also classify the irreducible components of $\mathcal{L}$ and show that they are smooth. Using some methods of M. Yakimov, we give a new description and proof of Karolinsky's classification of the diagonal $G$-orbits in $\mathcal{L}$, which, as a special case, recovers the Belavin-Drinfeld classification of quasi-triangular $\mathfrak{r}$-matrices on $\mathfrak{g}$. Furthermore, $\mathcal{L}$ has a canonical Poisson structure, and we compute its rank at each point and describe its symplectic leaf decomposition in terms of intersections of orbits of two subgroups of $G \times G$.

(c) 2006 Elsevier SAS

RÉSUMÉ. - Motivé par le théorème de Drinfeld sur les espaces de Poisson homogènes, nous étudions la variété $\mathcal{L}$ des sous-algèbres de Lie lagrangiennes de $\mathfrak{g} \oplus \mathfrak{g}$ pour $\mathfrak{g}$, une algèbre de Lie complexe semisimple. Soit $G$ le groupe adjoint de $\mathfrak{g}$. Nous montrons que les adhérences des $(G \times G)$-orbites dans $\mathcal{L}$ sont les variétés sphériques et lisses. Aussi, nous classifions les composantes irréductibles de $\mathcal{L}$ et nous montrons qu'elles sont lisses. Nous employons des méthodes de M. Yakimov pour donner une nouvelle description et une nouvelle preuve de la classification de Karolinsky des orbites diagonales de $G$ dans $\mathcal{L}$, qui, comme cas spécial, donne la classification de Belavin-Drinfeld des $\mathfrak{r}$-matrices quasi-triangulaires de $\mathfrak{g}$. En outre, $\mathcal{L}$ possède une structure de Poisson canonique, et nous calculons son rang à chaque point et nous décrivons sa décomposition en feuilles symplectiques en terme des intersections des orbites des deux sous-groupes de $G \times G$.

(c) 2006 Elsevier SAS

\section{Contents}

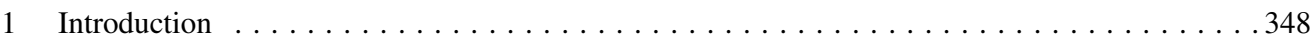

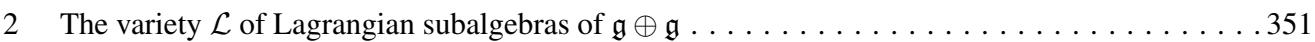

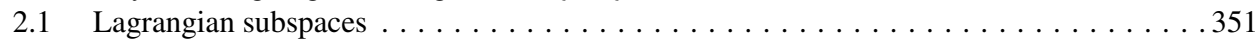

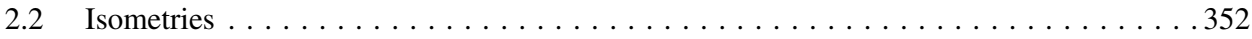

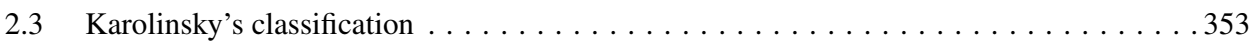

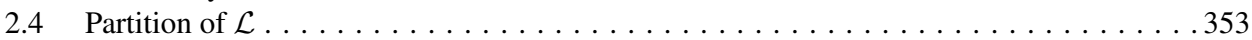

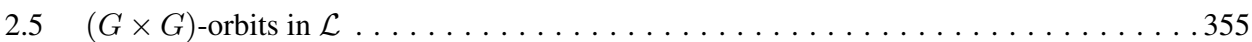

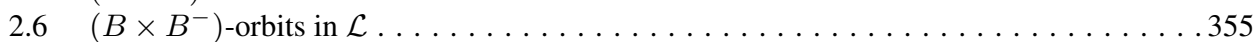

2.7 The De Concini-Procesi compactifications $Z_{d}(G)$ of $G \ldots \ldots \ldots \ldots \ldots \ldots \ldots$

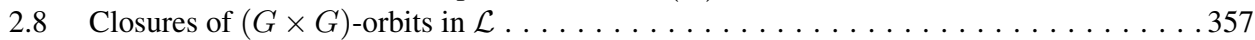

2.9 The geometry of the strata $\mathcal{L}^{\varepsilon}(S, T, d) \ldots \ldots \ldots \ldots \ldots \ldots \ldots \ldots \ldots \ldots \ldots$

2.10 The geometry of the closure of $\overline{\mathcal{L}^{\varepsilon}(S, T, d)} \ldots \ldots \ldots \ldots \ldots \ldots \ldots \ldots \ldots$

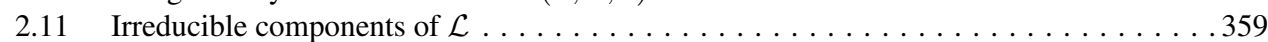

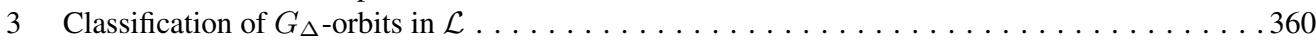

ANNALES SCIENTIFIQUES DE L'ÉCOLE NORMALE SUPÉRIEURE 0012-9593/02/@ 2006 Elsevier SAS. All rights reserved. 


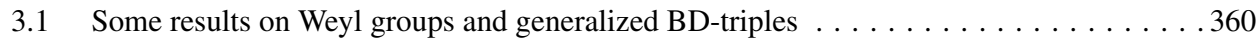

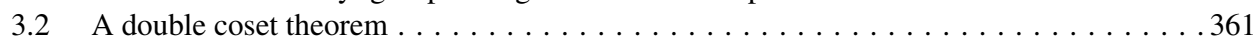

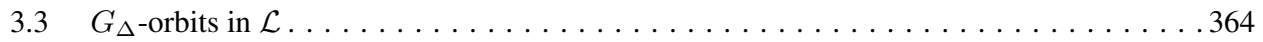

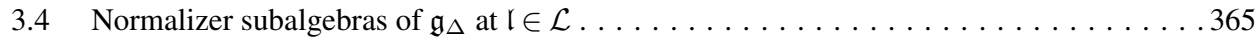

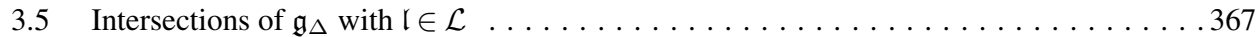

3.6 Examples of smooth $G_{\Delta}$-orbit closures in $\mathcal{L} \ldots \ldots \ldots \ldots \ldots \ldots$

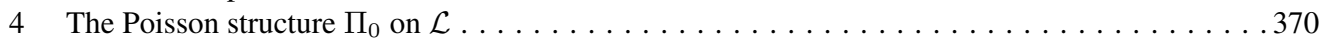

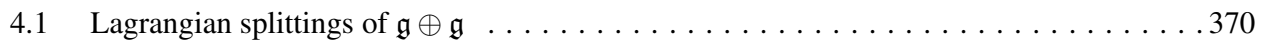

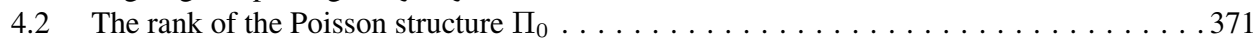

4.3 The action of $H_{\Delta}$ on the set of symplectic leaves of the Poisson structure $\Pi_{0} \ldots \ldots 374$

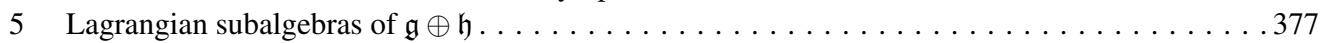

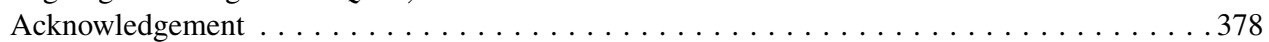

References ..................................... 378

\section{Introduction}

Let $\mathfrak{d}$ be a $2 n$-dimensional Lie algebra over $k=\mathbb{R}$ or $\mathbb{C}$, together with a symmetric, nondegenerate, and ad-invariant bilinear form $\langle$,$\rangle . A Lie subalgebra \mathfrak{l}$ of $\mathfrak{d}$ is said to be Lagrangian if $\mathfrak{l}$ is maximal isotropic with respect to $\langle$, $\rangle$, i.e., if $\operatorname{dim}_{k} \mathfrak{l}=n$ and if $\langle x, y\rangle=0$ for all $x, y \in \mathfrak{l}$. By a Lagrangian splitting of $\mathfrak{d}$ we mean a direct sum decomposition $\mathfrak{d}=\mathfrak{l}_{1}+\mathfrak{l}_{2}$, where $\mathfrak{l}_{1}$ and $\mathfrak{l}_{2}$ are two Lagrangian subalgebras of $\mathfrak{d}$. Denote by $\mathcal{L}(\mathfrak{d})$ the set of all Lagrangian subalgebras of $\mathfrak{d}$. It is an algebraic subvariety of the $\operatorname{Grassmannian} \operatorname{Gr}(n, \mathfrak{d})$ of $n$-dimensional subspaces of $\mathfrak{d}$, and every connected Lie group $D$ with Lie algebra $\mathfrak{d}$ acts on $\mathcal{L}(\mathfrak{d})$ via the adjoint action of $D$ on $\mathfrak{d}$. We proved in [9] that each Lagrangian splitting $\mathfrak{d}=\mathfrak{l}_{1}+\mathfrak{l}_{2}$ gives rise to a Poisson structure $\Pi_{\mathfrak{l}_{1}, \mathfrak{l}_{2}}$ on $\mathcal{L}(\mathfrak{d})$, making $\mathcal{L}(\mathfrak{d})$ into a Poisson variety. Moreover, if $L_{1}$ and $L_{2}$ are the connected subgroups of $D$ with Lie algebras $\mathfrak{l}_{1}$ and $\mathfrak{l}_{2}$ respectively, all the $L_{1}$ and $L_{2}$-orbits in $\mathcal{L}(\mathfrak{d})$ are Poisson submanifolds of $\Pi_{\mathfrak{l}_{1}, \mathfrak{l}_{2}}$.

The above construction in [9] was motivated by the work of Drinfeld [7] on Poisson homogeneous spaces. Indeed, a Lagrangian splitting $\mathfrak{d}=\mathfrak{l}_{1}+\mathfrak{l}_{2}$ of $\mathfrak{d}$ gives rise to the Manin triple $\left(\mathfrak{d}, \mathfrak{l}_{1}, \mathfrak{l}_{2}\right)$, which in turn defines Poisson structures $\pi_{1}$ and $\pi_{2}$ on $L_{1}$ and $L_{2}$ respectively, making them into Poisson Lie groups [19]. A Poisson space $(M, \pi)$ is said to be $\left(L_{1}, \pi_{1}\right)$ homogeneous if $L_{1}$ acts on $M$ transitively and if the action map $L_{1} \times M \rightarrow M$ is Poisson. In [7], Drinfeld constructed an $L_{1}$-equivariant map $M \rightarrow \mathcal{L}(\mathfrak{d})$ for every $\left(L_{1}, \pi_{1}\right)$-homogeneous Poisson space $(M, \pi)$ and proved that $\left(L_{1}, \pi_{1}\right)$-homogeneous Poisson spaces correspond to $L_{1}$ orbits in $\mathcal{L}(\mathfrak{d})$ in this way. The Poisson structure $\Pi_{\mathfrak{l}_{1}, \mathfrak{l}_{2}}$ on $\mathcal{L}(\mathfrak{d})$ is constructed in such a way that the Drinfeld map $M \rightarrow \mathcal{L}(\mathfrak{d})$ is a Poisson map. In many cases, the Drinfeld map $M \rightarrow \mathcal{L}(\mathfrak{d})$ is a local diffeomorphism onto its image. Thus we can think of $L_{1}$-orbits in $\mathcal{L}(\mathfrak{d})$ as models for $\left(L_{1}, \pi_{1}\right)$-homogeneous Poisson spaces. For this reason, it is interesting to study the geometry of the variety $\mathcal{L}(\mathfrak{d})$, the $L_{1}$ and $L_{2}$-orbits in $\mathcal{L}(\mathfrak{d})$, and the Poisson structures $\Pi_{\mathfrak{l}_{1}, \mathfrak{l}_{2}}$ on $\mathcal{L}(\mathfrak{d})$.

There are many examples of Lie algebras $\mathfrak{d}$ with symmetric, non-degenerate, and ad-invariant bilinear forms. The geometry of $\mathcal{L}(\mathfrak{d})$ is different from case to case. Moreover, there can be many Lagrangian splittings for a given $\mathfrak{d}$, resulting in many Poisson structures on $\mathcal{L}(\mathfrak{d})$.

Example 1.1. - Let $\mathfrak{g}$ be a complex semi-simple Lie algebra and regard it as a real Lie algebra, and let $\langle$,$\rangle be the imaginary part of the Killing form of \mathfrak{g}$. The geometry of $\mathcal{L}(\mathfrak{g})$ was studied in [9]. In particular, we determined the irreducible components of $\mathcal{L}(\mathfrak{g})$ and classified the $G$-orbits in $\mathcal{L}(\mathfrak{g})$, where $G$ is the adjoint group of $\mathfrak{g}$. Let $\mathfrak{g}=\mathfrak{k}+\mathfrak{a}+\mathfrak{n}$ be an Iwasawa decomposition of $\mathfrak{g}$. Then both $\mathfrak{k}$ and $\mathfrak{a}+\mathfrak{n}$ are Lagrangian, so $\mathfrak{g}=\mathfrak{k}+(\mathfrak{a}+\mathfrak{n})$ is a Lagrangian splitting, resulting in a Poisson structure on $\mathcal{L}(\mathfrak{g})$ which we denote by $\pi_{0}$. Many interesting Poisson manifolds appear as $G$ or $K$-orbits inside $\mathcal{L}(\mathfrak{g})$, where $K$ is the connected subgroup of $G$ with Lie algebra $\mathfrak{k}$. Among

$4^{\text {e }}$ SÉRIE - TOME $39-2006-\mathrm{N}^{\circ} 2$ 
such Poisson manifolds are the flag manifolds of $G$ and the compact symmetric spaces associated to real forms of $G$. Detailed studies of the Poisson geometry of these Poisson structures and some applications to Lie theory have been given in [22,23,8,11]. For example, a flag manifold $X$ of $G$ can be identified with a certain $K$-orbit in $\mathcal{L}(\mathfrak{g})$. The resulting Poisson structure $\pi_{0}$ on $X$ is called the Bruhat-Poisson structure because its symplectic leaves are Bruhat cells in $X$. In [22] and [8], we established connections between the Poisson geometry of $\pi_{0}$ and the harmonic forms on $X$ constructed by Kostant [20] in 1963, and we gave a Poisson geometric interpretation of the Kostant-Kumar approach [21] to Schubert calculus on $X$.

Example 1.2. - Let $\mathfrak{g}$ be any $n$-dimensional Lie algebra, and let $\mathfrak{d}$ be the semi-direct product of $\mathfrak{g}$ and its dual space $\mathfrak{g}^{*}$. The symmetric bilinear form $\langle x+\xi, y+\eta\rangle=\langle x, \eta\rangle+\langle y, \xi\rangle$ for $x, y \in \mathfrak{g}$ and $\xi, \eta \in \mathfrak{g}^{*}$ is non-degenerate and ad-invariant. When $\mathfrak{g}$ is semi-simple, Lagrangian subalgebras of $\mathfrak{d}$ are not easy to classify (except for low dimensional cases), for, as a subproblem, one needs to classify all abelian subalgebras of $\mathfrak{g}$. See $[19,16]$, and their references for more detail. The description of the geometry of $\mathcal{L}(\mathfrak{d})$ in this case is an open problem.

In this paper, we consider the complexification of Example 1.1. Namely, let $\mathfrak{g}$ be a complex semi-simple Lie algebra and $\mathfrak{d}=\mathfrak{g} \oplus \mathfrak{g}$ the direct sum Lie algebra with the bilinear form

$$
\left\langle\left(x_{1}, x_{2}\right),\left(y_{1}, y_{2}\right)\right\rangle=\left\langle\left\langle x_{1}, y_{1}\right\rangle\right\rangle-\left\langle\left\langle x_{2}, y_{2}\right\rangle\right\rangle, \quad x_{1}, x_{2}, y_{1}, y_{2} \in \mathfrak{g},
$$

where $\langle$,$\rangle is a fixed symmetric, non-degenerate, and ad-invariant bilinear form on \mathfrak{g}$. The variety of Lagrangian subalgebras of $\mathfrak{d}$ with respect to $\langle$,$\rangle will be denoted by \mathcal{L}$.

The classification of Lagrangian subalgebras of $\mathfrak{d}$ has been given by Karolinsky [15], and Lagrangian splittings of $\mathfrak{g} \oplus \mathfrak{g}$ have been classified by Delorme [6]. In this paper, we establish the first few steps in the study of the Poisson structures on $\mathcal{L}$ defined by Lagrangian splittings of $\mathfrak{g} \oplus \mathfrak{g}$. Namely, we will first describe the geometry of $\mathcal{L}$ in the following terms:

(1) the $(G \times G)$-orbits in $\mathcal{L}$ and their closures, where $G$ is the adjoint group of $\mathfrak{g}$;

(2) the irreducible components of $\mathcal{L}$;

We then look at the Poisson structure $\Pi_{0}$ on $\mathcal{L}$ defined by the so-called standard Lagrangian splitting $\mathfrak{d}=\mathfrak{g}_{\Delta}+\mathfrak{g}_{\mathrm{st}}^{*}$, where $\mathfrak{g}_{\Delta}=\{(x, x): x \in \mathfrak{g}\}$ and $\mathfrak{g}_{\mathrm{st}}^{*} \subset \mathfrak{b} \oplus \mathfrak{b}^{-}$, where $\mathfrak{b}$ and $\mathfrak{b}^{-}$are two opposite Borel subalgebras of $\mathfrak{g}$. Let $G_{\Delta}=\{(g, g): g \in G\}$, and let $B$ and $B^{-}$be the Borel subgroups of $G$ with Lie algebras $\mathfrak{b}$ and $\mathfrak{b}^{-}$respectively. We will compute the rank of $\Pi_{0}$ in $\mathcal{L}$ and study the symplectic leaf decomposition of $\mathcal{L}$ with respect to $\Pi_{0}$ in terms of the intersections of $G_{\Delta}$ and $\left(B \times B^{-}\right)$-orbits in $\mathcal{L}$.

We regard the Poisson structure $\Pi_{0}$ as the most important Poisson structure on $\mathcal{L}$, especially since it is more closely related to applications to other areas of mathematics. The study of the symplectic leaf decomposition of the Poisson structure $\Pi_{\mathfrak{l}_{1}, \mathfrak{l}_{2}}$ defined by an arbitrary Lagrangian splitting $\mathfrak{g} \oplus \mathfrak{g}=\mathfrak{l}_{1}+\mathfrak{l}_{2}$ will be carried out in [25] using results of [24]. This analysis is much more technically involved, so we think it is worthwhile to present the important special case of $\Pi_{0}$ separately. In particular, in computing the rank of $\Pi_{0}$ in $\mathcal{L}$, we need to classify $G_{\Delta}$-orbits in $\mathcal{L}$ and compute the normalizer subalgebra of $\mathfrak{g}_{\Delta}$ for every $\mathfrak{l} \in \mathcal{L}$. The classification of $G_{\Delta^{-}}$ orbits in $\mathcal{L}$ follows directly from a special case of Theorem 2.2 in [24]. However, the inductive procedure used in the proof of Theorem 2.2 in [24] is rather involved. To make the paper selfcontained and more comprehensible, we present a shorter inductive proof for the classification of $G_{\Delta}$-orbits in $\mathcal{L}$. Our proof and the one in [24] are both adapted from [33]. The description of the normalizer subalgebra of $\mathfrak{g}_{\Delta}$ at $\mathfrak{l} \in \mathcal{L}$ can be derived from Theorem 2.5 in [24]. Again for the purpose of completeness and comprehensibility, we give a simple and more direct computation of these subalgebras. 
We point out that E. Karolinsky has in [15] given a classification of $G_{\Delta}$-orbits in $\mathcal{L}$ in different terms. Our classification is more in line with that of Lagrangian splittings in [6], and in particular, the Belavin-Drinfeld theorem [3] on Lagrangian splittings of the form $\mathfrak{g} \oplus \mathfrak{g}=\mathfrak{g}_{\Delta}+\mathfrak{l}$ follows easily from our classification, but does not seem to be easily derived from Karolinsky's classification. A special case of our classification is the classification of $G_{\Delta}$-orbits on the wonderful compactification of $G$, and this special case was first determined by Lusztig in [26] and [27], where it is used to develop a theory of character sheaves for the compactifications. Our arguments are somewhat different from those in [26] and [27]. It would be very interesting to find connections between Poisson geometry and character sheaves.

We now give more details of the main results in this paper:

Following O. Schiffmann [28], we define a generalized Belavin-Drinfeld triple (generalized BD-triple) to be a triple $(S, T, d)$, where $S$ and $T$ are two subsets of the set $\Gamma$ of vertices of the Dynkin diagram of $\mathfrak{g}$, and $d: S \rightarrow T$ is an isometry with respect to $\langle$,$\rangle . For a generalized$ BD-triple $(S, T, d)$, let $P_{S}$ and $P_{T}^{-}$be respectively the standard parabolic subgroups of $G$ of type $S$ and opposite type $T$ (see Notation 2.11) with Levi decompositions $P_{S}=M_{S} N_{S}$ and $P_{T}^{-}=M_{T} N_{T}^{-}$. Let $G_{S}$ and $G_{T}$ be the quotients of $M_{S}$ and $M_{T}$ by their respective centers, and let $\chi_{S}: M_{S} \rightarrow G_{S}$ and $\chi_{T}: M_{T} \rightarrow G_{T}$ be the natural projections. Let $\gamma_{d}: G_{S} \rightarrow G_{T}$ be a group isomorphism induced by $d$, and define the subgroup $R_{S, T, d}$ of $P_{S} \times P_{T}^{-}$by

$$
R_{S, T, d}=\left\{\left(m_{S}, m_{T}\right) \in M_{S} \times M_{T}: \gamma_{d}\left(\chi_{S}\left(m_{S}\right)\right)=\chi_{T}\left(m_{T}\right)\right\}\left(N_{S} \times N_{T}^{-}\right) .
$$

In Section 2, we establish the following facts on $(G \times G)$-orbits and their closures in $\mathcal{L}$ (Proposition 2.17, Corollary 2.22, and Proposition 2.25):

(1) Every $(G \times G)$-orbit in $\mathcal{L}$ is isomorphic to $(G \times G) / R_{S, T, d}$ for a generalized BD-triple $(S, T, d)$, so the $(G \times G)$-orbit types in $\mathcal{L}$ correspond bijectively to generalized BD-triples for $G$; Every $(G \times G)$-orbit in $\mathcal{L}$ is a $(G \times G)$-spherical homogeneous space.

(2) For a generalized BD-triple $(S, T, d)$, the closure of a $(G \times G)$-orbit of type $(S, T, d)$ is a fiber bundle over the flag manifold $G / P_{S} \times G / P_{T}^{-}$whose fiber is isomorphic to a De Concini-Procesi compactification of $G_{S}$.

We also study in Section 2 the irreducible components of $\mathcal{L}$. We prove (Corollary 2.27, Theorems 2.29 and 2.32):

(1) The irreducible components of $\mathcal{L}$ are roughly (see Theorem 2.32 for detail) labeled by quadruples $(S, T, d, \varepsilon)$, where $(S, T, d)$ are generalized $B D$-triples and $\varepsilon \in\{0,1\}$;

(2) The irreducible component corresponding to $(S, T, d, \varepsilon)$ is a fiber bundle over the flag manifold $G / P_{S} \times G / P_{T}^{-}$with fiber isomorphic to the product of a De Concini-Procesi compactification of $G_{S}$ and a homogeneous space of a special orthogonal group. In particular, all the irreducible components of $\mathcal{L}$ are smooth;

(3) $\mathcal{L}$ has two connected components.

In Section 3, we give the classification of $G_{\Delta}$-orbits in $\mathcal{L}$, and we compute the normalizer subalgebra of $\mathfrak{g}_{\Delta}$ at every $\mathfrak{l} \in \mathcal{L}$. In Section 4 , we compute the rank of the Poisson structure $\Pi_{0}$. In particular, we prove (Theorems 4.5 and 4.14 ):

Every non-empty intersection of a $G_{\Delta}$-orbit $\mathcal{O}$ and $a\left(B \times B^{-}\right)$-orbit $\mathcal{O}^{\prime}$ in $\mathcal{L}$ is a regular Poisson variety with respect to $\Pi_{0}$; The Cartan subgroup $H_{\Delta}=\{(h, h): h \in H\}$ of $G_{\Delta}$, where $H=B \cap B^{-}$, acts transitively on the set of symplectic leaves in $\mathcal{O} \cap \mathcal{O}^{\prime}$.

An interesting Poisson subvariety of $\left(\mathcal{L}, \Pi_{0}\right)$ is the De Concini-Procesi compactification $Z_{1}(G)$ of $G$ as the closure of the $(G \times G)$-orbit through $\mathfrak{g}_{\Delta} \in \mathcal{L}$. Conjugacy classes in $G$ and their closures in $Z_{1}(G)$ are all Poisson subvarieties of $\left(Z_{1}(G), \Pi_{0}\right)$. In particular, $\Pi_{0}$ restricted to a conjugacy class $C$ in $G$ is non-degenerate precisely on the intersection of $C$ with the open Bruhat cell $B^{-} B$ (see Corollary 4.7). It will be particularly interesting to compare the 
Poisson structure $\Pi_{0}$ on the unipotent variety in $G$ with the Kirillov-Kostant structure on the nilpotent cone in $\mathfrak{g}^{*}$. Another interesting Poisson subvariety of $\left(\mathcal{L}, \Pi_{0}\right)$ is the De Concini-Procesi compactification $X_{\sigma}$ of a complex symmetric space $G / G^{\sigma}$ for an involutive automorphism $\sigma$ of $G$ (Proposition 3.21). Intersections of $G_{\Delta}$-orbits and $\left(B \times B^{-}\right)$-orbits inside the closed $(G \times G)$-orbits in $\mathcal{L}$ are related to double Bruhat cells in $G$ (see Example 4.9), and Kogan and Zelevinsky [18] have constructed toric charts on some of the symplectic leaves in these closed orbits. It would be interesting to see how their methods can be applied to other symplectic leaves of $\Pi_{0}$.

\section{The variety $\mathcal{L}$ of Lagrangian subalgebras of $\mathfrak{g} \oplus \mathfrak{g}$}

Throughout this paper, $\mathfrak{g}$ will be an $n$-dimensional complex semi-simple Lie algebra, and $\langle$, $\rangle$ will be a fixed symmetric and non-degenerate ad-invariant bilinear form on $\mathfrak{g}$. Equip the direct product Lie algebra $\mathfrak{g} \oplus \mathfrak{g}$ with the symmetric non-degenerate ad-invariant bilinear form

$$
\left\langle\left(x_{1}, x_{2}\right),\left(y_{1}, y_{2}\right)\right\rangle=\left\langle\left\langle x_{1}, y_{1}\right\rangle\right\rangle-\left\langle\left\langle x_{2}, y_{2}\right\rangle\right\rangle, \quad x_{1}, x_{2}, y_{1}, y_{2} \in \mathfrak{g} .
$$

A Lagrangian subalgebra of $\mathfrak{g} \oplus \mathfrak{g}$ is by definition an $n$-dimensional complex Lie subalgebra of $\mathfrak{g} \oplus \mathfrak{g}$ that is isotropic with respect to $\langle$,$\rangle .$

NOTATION 2.1. - We will denote by $\mathcal{L}$ the variety of all Lagrangian subalgebras of $\mathfrak{g} \oplus \mathfrak{g}$ and by $\mathcal{L}_{\text {space }}(\mathfrak{g} \oplus \mathfrak{g})$ the variety of all $n$-dimensional isotropic subspaces of $\mathfrak{g} \oplus \mathfrak{g}$.

Let $G$ be the adjoint group of $\mathfrak{g}$. In this section, we will use the classification of Lagrangian subalgebras of $\mathfrak{g} \oplus \mathfrak{g}$ by Karolinsky [15] to study the $(G \times G)$-orbits and their closures in $\mathcal{L}$, and we will determine the irreducible components of $\mathcal{L}$.

\subsection{Lagrangian subspaces}

Let $U$ be a finite-dimensional complex vector space with a symmetric and non-degenerate bilinear form $\langle$,$\rangle . A subspace V$ of $U$ is said to be Lagrangian if $V$ is maximal isotropic with respect to $\langle$,$\rangle . If \operatorname{dim} U=2 n$ or $2 n+1$, Witt's theorem says that the dimension of a Lagrangian subspace of $U$ is $n$. Denote by $\mathcal{L}_{\text {space }}(U)$ the set of Lagrangian subspaces of $U$. It is a closed algebraic subvariety of $\operatorname{Gr}(n, U)$, the Grassmannian of $n$-dimensional subspaces of $U$.

Proposition 2.2. - (Arbarello et al. [1, pp. 102-103].) Let $\operatorname{dim} U=2 n$ (respectively $2 n+1)$ with $n>0$. Then $\mathcal{L}_{\text {space }}(U)$ is a smooth algebraic subvariety of $\operatorname{Gr}(n, U)$ with two (respectively one) connected components, each of which is isomorphic to $S O(2 n, \mathbb{C}) / P$ (respectively $S O(2 n+1, \mathbb{C}) / P$ ) where $P$ has Levi factor isomorphic to $G L(n, \mathbb{C})$. Moreover, $\mathcal{L}_{\text {space }}(U)$ has complex dimension $\frac{n(n-1)}{2}$ (respectively $\frac{n(n+1)}{2}$ ). When $\operatorname{dim} U=2 n$, two Lagrangian subspaces $V_{1}$ and $V_{2}$ are in the same connected component of $\mathcal{L}_{\text {space }}(U)$ if and only if $\operatorname{dim}\left(V_{1}\right)-\operatorname{dim}\left(V_{1} \cap V_{2}\right)$ is even.

NotATION 2.3. - For $U=\mathfrak{g} \oplus \mathfrak{g}$ with the bilinear form $\langle$,$\rangle in (2.1), let \mathcal{L}^{0}$ be the intersection of $\mathcal{L}$ with the connected component of $\mathcal{L}_{\text {space }}(\mathfrak{g} \oplus \mathfrak{g})$ containing the diagonal of $\mathfrak{g} \oplus \mathfrak{g}$. The intersection of $\mathcal{L}$ with the other connected component of $\mathcal{L}_{\text {space }}(\mathfrak{g} \oplus \mathfrak{g})$ will be denoted by $\mathcal{L}^{1}$.

Let $\mathfrak{h}$ be a Cartan subalgebra of $\mathfrak{g}$, and let $\mathfrak{n}$ and $\mathfrak{n}^{-}$be the opposite nilpotent subalgebras of $\mathfrak{g}$ corresponding to a choice of positive roots for $(\mathfrak{g}, \mathfrak{h})$. For a Lagrangian subspace $V$ of $\mathfrak{h} \oplus \mathfrak{h}$ with respect to $\langle$,$\rangle , let \mathfrak{l}_{V}=V+\left\{(x, y): x \in \mathfrak{n}, y \in \mathfrak{n}^{-}\right\}$. Then $\mathfrak{l}_{V} \in \mathcal{L}$. It is easy to see from Proposition 2.2 that $\mathfrak{l}_{V_{1}}$ and $\mathfrak{l}_{V_{2}}$ are in the same connected component of $\mathcal{L}_{\text {space }}(\mathfrak{g} \oplus \mathfrak{g})$ if and 
only if $V_{1}$ and $V_{2}$ are in the same connected component of $\mathcal{L}_{\text {space }}(\mathfrak{h} \oplus \mathfrak{h})$. In particular, $\mathcal{L}^{1}$ is non-empty.

\subsection{Isometries}

We collect some results on automorphisms that will be used in later sections.

Notation 2.4. - Throughout this paper, we fix a Cartan subalgebra $\mathfrak{h}$ and a choice $\Sigma^{+}$of positive roots in the set $\Sigma$ of all roots of $\mathfrak{g}$ relative to $\mathfrak{h}$, and let $\mathfrak{g}=\mathfrak{h}+\sum_{\alpha \in \Sigma} \mathfrak{g}_{\alpha}$ be the root decomposition. Let $\Gamma$ be the set of simple roots in $\Sigma^{+}$. For $\alpha \in \Sigma$, let $H_{\alpha} \in \mathfrak{h}$ be such that $\left\langle\left\langle H_{\alpha}, H\right\rangle=\alpha(H)\right.$ for all $H \in \mathfrak{h}$. For $\alpha \in \Sigma^{+}$, fix root vectors $E_{\alpha} \in \mathfrak{g}_{\alpha}$ and $E_{-\alpha} \in \mathfrak{g}_{-\alpha}$ such that $\left\langle\left\langle E_{\alpha}, E_{-\alpha}\right\rangle\right\rangle=1$. For a subset $S$ of $\Gamma$, let $[S]$ be the set of roots in the linear span of $S$, and let $G_{S}$ be the adjoint group of the semi-simple Lie algebra $\mathfrak{g}_{S}$ given by

$$
\mathfrak{g}_{S}=\operatorname{span}_{\mathbb{C}}\left\{H_{\alpha}: \alpha \in S\right\}+\sum_{\alpha \in[S]} \mathfrak{g}_{\alpha} \subset \mathfrak{g}
$$

For $S, T \subset \Gamma$, we are interested in Lie algebra isomorphisms $\mathfrak{g}_{S} \rightarrow \mathfrak{g}_{T}$ that preserve the restrictions of $\langle\langle\rangle$,$\rangle to \mathfrak{g}_{S}$ and $\mathfrak{g}_{T}$. We will simply refer to this property as preserving $\langle\langle$,$\rangle .$

Definition 2.5. - Let $S, T \subset \Gamma$. By an isometry from $S$ to $T$ we mean a bijection $d: S \rightarrow T$ such that $\langle\langle d \alpha, d \beta\rangle\rangle=\langle\langle\alpha, \beta\rangle\rangle$ for all $\alpha, \beta \in S$, where $\langle\langle\alpha, \beta\rangle\rangle=\left\langle\left\langle H_{\alpha}, H_{\beta}\right\rangle\right\rangle$. Let $I(S, T)$ be the set of all isometries from $S$ to $T$. Following [28], a triple $(S, T, d)$, where $S, T \subset \Gamma$ and $d \in I(S, T)$, will also be called a generalized Belavin-Drinfeld (generalized BD-)triple for $G$.

LEMMA 2.6. - For any $S, T \subset \Gamma$ and $d \in I(S, T)$, there is a unique isomorphism $\gamma_{d}: \mathfrak{g}_{S} \rightarrow \mathfrak{g}_{T}$ such that

$$
\gamma_{d}\left(E_{\alpha}\right)=E_{d(\alpha)}, \quad \gamma_{d}\left(H_{\alpha}\right)=H_{d(\alpha)}, \quad \forall \alpha \in S .
$$

Moreover, $\gamma_{d}$ preserves $\left\langle\langle\right.$,$\rangle , and for every Lie algebra isomorphism \mu: \mathfrak{g}_{S} \rightarrow \mathfrak{g}_{T}$ preserving $\langle$,$\rangle , there is a unique d \in I(S, T)$ and a unique $g \in G_{S}$ such that $\mu=\gamma_{d} \operatorname{Ad}_{g}$.

Proof. - Existence and uniqueness of $\gamma_{d}$ is by Theorem 2.108 in [17]. For $\alpha \in \Sigma^{+}$, let $\lambda_{\alpha}, \mu_{\alpha} \in \mathbb{C}$ be such that $\gamma_{d}\left(E_{\alpha}\right)=\lambda_{\alpha} E_{d \alpha}$ and $\gamma_{d}\left(E_{-\alpha}\right)=\mu_{\alpha} E_{-d \alpha}$. By applying $\gamma_{d}$ to the identity $\left[E_{\alpha}, E_{-\alpha}\right]=H_{\alpha}$ we get $\lambda_{\alpha} \mu_{\alpha}=1$ for every $\alpha \in \Sigma^{+}$. It follows that $\gamma_{d}$ preserves $\langle$,$\rangle . Suppose that \mu: \mathfrak{g}_{S} \rightarrow \mathfrak{g}_{T}$ is a Lie algebra isomorphism preserving $\langle$,$\rangle . Let d_{1}$ be any isomorphism from the Dynkin diagram of $\mathfrak{g}_{S}$ to the Dynkin diagram of $\mathfrak{g}_{T}$. Let $\gamma_{d_{1}}: \mathfrak{g}_{S} \rightarrow \mathfrak{g}_{T}$ be defined as in (2.2). Then $\nu:=\gamma_{d_{1}}^{-1} \mu$ is an automorphism of $\mathfrak{g}_{S}$. Recall that there is a short exact sequence

$$
1 \rightarrow G_{S} \rightarrow \operatorname{Aut}_{\mathfrak{g}_{S}} \rightarrow \mathrm{Aut}_{S} \rightarrow 1
$$

where $\mathrm{Aut}_{\mathfrak{g}_{S}}$ is the group of automorphisms of $\mathfrak{g}_{S}$, and Aut $\mathrm{t}_{S}$ is the group of automorphisms of the Dynkin diagram of $\mathfrak{g}_{S}$. Let $d_{2} \in \mathrm{Aut}_{S}$ be the image of $\nu$ under the map Aut $\mathfrak{g}_{S} \rightarrow \operatorname{Aut}_{S}$ and write $\nu=\gamma_{d_{2}} \operatorname{Ad}_{g}$ for some $g \in G_{S}$. Thus $\mu=\gamma_{d_{1}} \gamma_{d_{2}} \operatorname{Ad}_{g}=\gamma_{d_{1} d_{2}} \operatorname{Ad}_{g}$. Since $\mu$ and $\operatorname{Ad}_{g}$ are isometries of $\left\langle\langle\right.$,$\rangle , so is \gamma_{d_{1} d_{2}}$. Thus $d:=d_{1} d_{2} \in I(S, T)$ is an isometry, and $\mu=\gamma_{d} \operatorname{Ad}_{g}$.

Uniqueness of $d$ and $g$ follows from the fact that if $g_{0} \in G_{S}$ preserves a Cartan subalgebra and acts as the identity on all simple root spaces, then $g_{0}$ is the identity element.

Definition 2.7. - A $\langle$, $\rangle$-preserving Lie algebra isomorphism $\mu: \mathfrak{g}_{S} \rightarrow \mathfrak{g}_{T}$ is said to be of type $d$ for $d \in I(S, T)$ if $d$ is the unique element in $I(S, T)$ such that $\mu=\gamma_{d} \operatorname{Ad}_{g}$ for some $g \in G_{S}$.

$4^{\mathrm{e}}$ SÉRIE - TOME $39-2006-\mathrm{N}^{\circ} 2$ 


\subsection{Karolinsky's classification}

Karolinsky [15] has classified the Lagrangian subalgebras of $\mathfrak{g} \oplus \mathfrak{g}$ with respect to the bilinear form $\langle$,$\rangle given in (2.1). We recall his results now.$

NotATion 2.8. - For a parabolic subalgebra $\mathfrak{p}$ of $\mathfrak{g}$, let $\mathfrak{n}$ be its nilradical and $\mathfrak{m}:=\mathfrak{p} / \mathfrak{n}$ its Levi factor. Let $\mathfrak{m}=[\mathfrak{m}, \mathfrak{m}]+\mathfrak{z}$ be the decomposition of $\mathfrak{m}$ into the direct sum of its derived algebra $[\mathfrak{m}, \mathfrak{m}]$ and its center $\mathfrak{z}$. Recall that $[\mathfrak{m}, \mathfrak{m}]$ is semi-simple and that the restrictions of the bilinear form $\left\langle\langle\right.$,$\rangle to \mathfrak{m}$ and $\mathfrak{z}$ are both non-degenerate. If $\mathfrak{p}^{\prime}$ is another parabolic subalgebra, denote its nilradical, Levi factor, and center of Levi factor, etc. by $\mathfrak{n}^{\prime}, \mathfrak{m}^{\prime}$, and $\mathfrak{z}^{\prime}$, etc. When speaking of Lagrangian subspaces of $\mathfrak{z} \oplus \mathfrak{z}^{\prime}$, we mean with respect to the restriction of $\langle$,$\rangle to$ $\mathfrak{z} \oplus \mathfrak{z}^{\prime}$.

Definition 2.9. - A quadruple $\left(\mathfrak{p}, \mathfrak{p}^{\prime}, \mu, V\right)$ is called admissible if $\mathfrak{p}$ and $\mathfrak{p}^{\prime}$ are parabolic subalgebras of $\mathfrak{g}, \mu:[\mathfrak{m}, \mathfrak{m}] \rightarrow\left[\mathfrak{m}^{\prime}, \mathfrak{m}^{\prime}\right]$ is a Lie algebra isomorphism preserving $\langle$,$\rangle , and V$ is a Lagrangian subspace of $\mathfrak{z} \oplus \mathfrak{z}^{\prime}$.

If $\left(\mathfrak{p}, \mathfrak{p}^{\prime}, \mu, V\right)$ is admissible, set

$$
\mathfrak{l}\left(\mathfrak{p}, \mathfrak{p}^{\prime}, \mu, V\right):=\left\{\left(x, x^{\prime}\right): x \in \mathfrak{p}, x^{\prime} \in \mathfrak{p}^{\prime}, \mu\left(x_{[\mathfrak{m}, \mathfrak{m}]}\right)=x_{\left[\mathfrak{m}^{\prime}, \mathfrak{m}^{\prime}\right]}^{\prime},\left(x_{\mathfrak{z}}, x_{\mathfrak{z}^{\prime}}^{\prime}\right) \in V\right\} \subset \mathfrak{g} \oplus \mathfrak{g},
$$

where for $x \in \mathfrak{p}, x_{[\mathfrak{m}, \mathfrak{m}]} \in[\mathfrak{m}, \mathfrak{m}]$ and $x_{\mathfrak{z}} \in \mathfrak{z}$ are respectively the $[\mathfrak{m}, \mathfrak{m}]$ - and $\mathfrak{z}$-components of $x+\mathfrak{n} \in \mathfrak{p} / \mathfrak{n}=[\mathfrak{m}, \mathfrak{m}]+\mathfrak{z}$. We use similar notation for $x^{\prime} \in \mathfrak{p}^{\prime}$.

THEOREM 2.10.- (See [15].) $\mathfrak{l}\left(\mathfrak{p}, \mathfrak{p}^{\prime}, \mu, V\right)$ is a Lagrangian subalgebra if $\left(\mathfrak{p}, \mathfrak{p}^{\prime}, \mu, V\right)$ is admissible, and every Lagrangian subalgebra of $\mathfrak{g} \oplus \mathfrak{g}$ is of the form $\mathfrak{l}\left(\mathfrak{p}, \mathfrak{p}^{\prime}, \mu, V\right)$ for some admissible quadruple.

\subsection{Partition of $\mathcal{L}$}

NOTATION 2.11. - Recall the fixed choice $\Sigma^{+}$of positive roots from Notation 2.4. Set

$$
\mathfrak{n}=\sum_{\alpha \in \Sigma^{+}} \mathfrak{g}_{\alpha}, \quad \mathfrak{n}^{-}=\sum_{\alpha \in \Sigma^{+}} \mathfrak{g}_{-\alpha}
$$

A parabolic subalgebra $\mathfrak{p}$ of $\mathfrak{g}$ is said to be standard if it contains the Borel subalgebra $\mathfrak{b}:=\mathfrak{h}+\mathfrak{n}$. Recall also that, for $S \subset \Gamma,[S]$ denotes the set of roots in the linear span of $S$. Let

$$
\mathfrak{m}_{S}=\mathfrak{h}+\sum_{\alpha \in[S]} \mathfrak{g}_{\alpha}, \quad \mathfrak{n}_{S}=\sum_{\alpha \in \Sigma^{+}-[S]} \mathfrak{g}_{\alpha}, \quad \mathfrak{n}_{S}^{-}=\sum_{\alpha \in \Sigma^{+}-[S]} \mathfrak{g}_{-\alpha}
$$

and $\mathfrak{p}_{S}=\mathfrak{m}_{S}+\mathfrak{n}_{S}, \mathfrak{p}_{S}^{-}=\mathfrak{m}_{S}+\mathfrak{n}_{S}^{-}$. We will refer to $\mathfrak{p}_{S}$ as the standard parabolic subalgebra of $\mathfrak{g}$ defined by $S$ and $\mathfrak{p}_{S}^{-}$the opposite of $\mathfrak{p}_{S}$. A parabolic subalgebra of $\mathfrak{p}$ of $\mathfrak{g}$ is said to be of type $S$ if $\mathfrak{p}$ is conjugate to $\mathfrak{p}_{S}$ and of the opposite-type $S$ if $\mathfrak{p}$ is conjugate to $\mathfrak{p}_{S}^{-}$. Let $\mathfrak{g}_{S}=\left[\mathfrak{m}_{S}, \mathfrak{m}_{S}\right]$ as in Notation 2.4 and

$$
\mathfrak{h}_{S}=\mathfrak{h} \cap \mathfrak{g}_{S}=\operatorname{span}_{\mathbb{C}}\left\{H_{\alpha}: \alpha \in[S]\right\}, \quad \mathfrak{z} S=\{x \in \mathfrak{h}: \alpha(x)=0, \forall \alpha \in S\} .
$$

Then $\mathfrak{p}_{S}=\mathfrak{z}_{S}+\mathfrak{g}_{S}+\mathfrak{n}_{S}$ and $\mathfrak{p}_{S}^{-}=\mathfrak{z}_{S}+\mathfrak{g}_{S}+\mathfrak{n}_{S}^{-}$. The connected subgroups of $G$ with Lie algebras $\mathfrak{p}_{S}, \mathfrak{p}_{S}^{-}, \mathfrak{m}_{S}, \mathfrak{n}_{S}$ and $\mathfrak{n}_{S}^{-}$will be respectively denoted by $P_{S}, P_{S}^{-}, M_{S}, N_{S}$ and $N_{S}^{-}$. Correspondingly we have $P_{S}=M_{S} N_{S}$ and $P_{S}^{-}=M_{S} N_{S}^{-}$, where $M_{S} \cap N_{S}=\{e\}=M_{S} \cap N_{S}^{-}$. 
Recall that $G_{S}$ denotes the adjoint group of $\mathfrak{g}_{S}$. Let $\chi_{S}: P_{S} \rightarrow G_{S}$ be the composition of the projection from $P_{S}=M_{S} N_{S}$ to $M_{S}$ along $N_{S}$ and the projection $M_{S} \rightarrow G_{S}$. The similarly defined projection from $P_{S}^{-}$to $G_{S}$ will also be denoted by $\chi_{S}$.

Returning to the notation in 2.8 , we have

LEMMA-DeFINITION 2.12. - Let $\left(\mathfrak{p}, \mathfrak{p}^{\prime}, \mu\right)$ be a triple, where $\mathfrak{p}$ and $\mathfrak{p}^{\prime}$ are parabolic subalgebras of $\mathfrak{g}$, and $\mu:[\mathfrak{m}, \mathfrak{m}] \rightarrow\left[\mathfrak{m}^{\prime}, \mathfrak{m}^{\prime}\right]$ is a Lie algebra isomorphism preserving $\left.\left.《,\right\rangle\right\rangle$. Assume that $\mathfrak{p}$ is of type $S$ and $\mathfrak{p}^{\prime}$ is of opposite-type T. Let $g_{1}, g_{2} \in G$ be such that $\operatorname{Ad}_{g_{1}} \mathfrak{p}=\mathfrak{p}_{S}$ and $\operatorname{Ad}_{g_{2}} \mathfrak{p}^{\prime}=\mathfrak{p}_{T}^{-}$. Let $\overline{\operatorname{Ad}_{g_{1}}}$ and $\overline{\operatorname{Ad}_{g_{2}}}$ be the induced Lie algebra isomorphisms

$$
\overline{\operatorname{Ad}_{g_{1}}}:[\mathfrak{m}, \mathfrak{m}] \rightarrow \mathfrak{g}_{S}, \quad \overline{\operatorname{Ad}_{g_{2}}}:\left[\mathfrak{m}^{\prime}, \mathfrak{m}^{\prime}\right] \rightarrow \mathfrak{g}_{T} .
$$

If $\mu^{\prime}:=\overline{\operatorname{Ad}_{g_{2}}} \circ \mu \circ\left(\overline{\operatorname{Ad}_{g_{1}}}\right)^{-1}: \mathfrak{g}_{S} \rightarrow \mathfrak{g}_{T}$ is of type $d \in I(S, T)$ as in Definition 2.7, we say that $\left(\mathfrak{p}, \mathfrak{p}^{\prime}, \mu\right)$ is of type $(S, T, d)$. The type of $\left(\mathfrak{p}, \mathfrak{p}^{\prime}, \mu\right)$ is independent of the choice of $g_{1}$ and $g_{2}$.

Proof. - If $h_{1}$ and $h_{2}$ in $G$ are such that $\operatorname{Ad}_{h_{1}} \mathfrak{p}=\mathfrak{p}_{S}$ and $\operatorname{Ad}_{h_{2}} \mathfrak{p}^{\prime}=\mathfrak{p}_{T}^{-}$, then there exist $p_{S} \in P_{S}$ and $p_{T}^{-} \in P_{T}^{-}$such that $h_{1}=p_{S} g_{1}$ and $h_{2}=p_{T}^{-} g_{2}$. Thus

$$
\mu^{\prime \prime}:=\overline{\operatorname{Ad}_{h_{2}}} \circ \mu \circ\left(\overline{\operatorname{Ad}_{h_{1}}}\right)^{-1}=\overline{\operatorname{Ad}_{p_{T}^{-}}} \circ \mu^{\prime} \circ\left(\overline{\operatorname{Ad}_{p_{S}}}\right)^{-1} .
$$

The action of $\overline{\operatorname{Ad}_{p_{S}}}$ on $\mathfrak{g}_{S}$ is by definition the adjoint action of $\chi_{S}\left(p_{S}\right) \in G_{S}$ on $\mathfrak{g}_{S}$, and the same holds for the action of $\overline{\operatorname{Ad}_{p_{T}^{-}}}$on $\mathfrak{g}_{T}$. Thus by Definition 2.7, the two maps $\mu^{\prime}$ and $\mu^{\prime \prime}$ have the same type.

We are now ready to partition $\mathcal{L}$. Recall the definitions of $\mathcal{L}^{0}$ and $\mathcal{L}^{1}$ in Notation 2.3.

Definition 2.13. - Let $S, T \subset \Gamma, d \in I(S, T)$, and $\varepsilon \in\{0,1\}$. Define $\mathcal{L}^{\varepsilon}(S, T, d)$ to be the set of all Lagrangian subalgebras $\mathfrak{l}\left(\mathfrak{p}, \mathfrak{p}^{\prime}, \mu, V\right)$ such that

(1) $\mathfrak{r}\left(\mathfrak{p}, \mathfrak{p}^{\prime}, \mu, V\right) \in \mathcal{L}^{\varepsilon}$

(2) $\left(\mathfrak{p}, \mathfrak{p}^{\prime}, \mu\right)$ is of type $(S, T, d)$.

We say that $\mathfrak{l} \in \mathcal{L}$ is of type $(\varepsilon, S, T, d)$ if $\mathfrak{l} \in \mathcal{L}^{\varepsilon}(S, T, d)$.

It is clear that

$$
\mathcal{L}=\bigcup_{\varepsilon \in\{0,1\}} \bigcup_{S, T \subset \Gamma, d \in I(S, T)} \mathcal{L}^{\varepsilon}(S, T, d)
$$

is a disjoint union, and each $\mathcal{L}^{\varepsilon}(S, T, d)$ is invariant under $G \times G$. Set

$$
\mathfrak{n}_{S} \oplus \mathfrak{n}_{T}^{-}=\left\{(x, y): x \in \mathfrak{n}_{S}, y \in \mathfrak{n}_{T}^{-}\right\} \subset \mathfrak{g} \oplus \mathfrak{g},
$$

and for each $V \in \mathcal{L}_{\text {space }}\left(\mathfrak{z}_{S} \oplus \mathfrak{z}_{T}\right)$, set

$$
\mathfrak{l}_{S, T, d, V}=V+\left(\mathfrak{n}_{S} \oplus \mathfrak{n}_{T}^{-}\right)+\left\{\left(x, \gamma_{d}(x)\right): x \in \mathfrak{g}_{S}\right\} \in \mathcal{L} .
$$

Note that $\operatorname{dim} \mathfrak{z}_{S}=\operatorname{dim} \mathfrak{z}_{T}$ because $\mathfrak{h}_{S} \cong \mathfrak{h}_{T}$ and $\mathfrak{h}=\mathfrak{z}_{S}+\mathfrak{h}_{S}=\mathfrak{z}_{T}+\mathfrak{h}_{T}$ are direct sums.

LEMMA-DEFINITION 2.14. - For $V_{1}, V_{2} \in \mathcal{L}_{\text {space }}\left(\mathfrak{z}_{S} \oplus \mathfrak{z}_{T}\right), \mathfrak{l}_{S, T, d, V_{1}}$ and $\mathfrak{l}_{S, T, d, V_{2}}$ are in the same connected component of $\mathcal{L}_{\text {space }}(\mathfrak{g} \oplus \mathfrak{g})$ if and only if $V_{1}$ and $V_{2}$ are in the same connected component of $\mathcal{L}_{\text {space }}\left(\mathfrak{z}_{S} \oplus \mathfrak{z}_{T}\right)$. For $\varepsilon=\{0,1\}$, let

$$
\mathcal{L}_{\text {space }}^{\varepsilon}\left(\mathfrak{z}_{S} \oplus \mathfrak{z}_{T}\right)=\left\{V \in \mathcal{L}_{\text {space }}\left(\mathfrak{z}_{S} \oplus \mathfrak{z}_{T}\right): \mathfrak{l}_{S, T, d, V} \in \mathcal{L}^{\varepsilon}\right\} .
$$

$4^{\mathrm{e}}$ SÉRIE - TOME $39-2006-\mathrm{N}^{\circ} 2$ 
Proof. - The statement follows from Proposition 2.2 and the fact

$$
\operatorname{dim}\left(\mathfrak{l}_{S, T, d, V_{1}}\right)-\operatorname{dim}\left(\mathfrak{l}_{S, T, d, V_{1}} \cap \mathfrak{l}_{S, T, d, V_{2}}\right)=\operatorname{dim}\left(V_{1}\right)-\operatorname{dim}\left(V_{1} \cap V_{2}\right) .
$$

PROPOSITION 2.15. - For any generalized BD-triple $(S, T, d)$ and $\varepsilon \in\{0,1\}$,

$$
\mathcal{L}^{\varepsilon}(S, T, d)=\bigcup_{V \in \mathcal{L}_{\text {space }}^{\varepsilon}\left(\mathfrak{z}_{S} \oplus_{\mathfrak{z}} T\right)}(G \times G) \cdot l_{S, T, d, V} \quad \text { (disjoint union). }
$$

Proof. - By Definition 2.13, every $(G \times G)$-orbit in $\mathcal{L}^{\varepsilon}(S, T, d)$ passes through an $\mathfrak{l}_{S, T, d, V}$ for some $V \in \mathcal{L}_{\text {space }}^{\varepsilon}\left(\mathfrak{z}_{S} \oplus \mathfrak{z}_{T}\right)$. If $V_{1}, V \in \mathcal{L}_{\text {space }}^{\varepsilon}\left(\mathfrak{z}_{S} \oplus \mathfrak{z}_{T}\right)$ are such that $\mathfrak{l}_{S, T, d, V_{1}}=$ $\operatorname{Ad}_{\left(g_{1}, g_{2}\right)} \mathfrak{l}_{S, T, d, V}$, then $\left(g_{1}, g_{2}\right)$ normalizes $\mathfrak{n}_{S} \oplus \mathfrak{n}_{T}^{-}$, so $\left(g_{1}, g_{2}\right) \in P_{S} \times P_{T}^{-}$, and it follows that $V_{1}=V$.

\section{5. $(G \times G)$-orbits in $\mathcal{L}$}

The following theorem follows immediately from Proposition 2.15 and the decomposition of $\mathcal{L}$ in (2.3).

THEOREM 2.16. - Every $(G \times G)$-orbit in $\mathcal{L}$ passes through an $\mathfrak{l}_{S, T, d, V}$ for a unique quadruple $(S, T, d, V)$, where $S, T \subset \Gamma, d \in I(S, T)$ and $V \in \mathcal{L}_{\text {space }}\left(\mathfrak{z}_{S} \oplus \mathfrak{z}_{T}\right)$.

For each $S, T \subset \Gamma$ and $d \in I(S, T)$, define the group (see Notation 2.11)

$$
R_{S, T, d}:=\left\{\left(p_{S}, p_{T}^{-}\right) \in P_{S} \times P_{T}^{-}: \gamma_{d}\left(\chi_{S}\left(p_{S}\right)\right)=\chi_{T}\left(p_{T}^{-}\right)\right\} \subset P_{S} \times P_{T}^{-} .
$$

Proposition 2.17. - Let $S, T \subset \Gamma, d \in I(S, T)$, and $V \in \mathcal{L}_{\text {space }}\left(\mathfrak{z}_{S} \oplus \mathfrak{z}_{T}\right)$.

(1) The $(G \times G)$-orbit in $\mathcal{L}$ through $\mathfrak{l}_{S, T, d, V}$ is isomorphic to $(G \times G) / R_{S, T, d}$ and it has dimension $n-z$, where $n=\operatorname{dim} \mathfrak{g}$ and $z=\operatorname{dim} \mathfrak{z}_{S}$.

(2) $(G \times G) \cdot \mathfrak{l}_{S, T, d, V}$ fibers over $G / P_{S} \times G / P_{T}^{-}$with fiber isomorphic to $G_{S}$.

Proof. - It is routine to check that the stabilizer of $\mathfrak{l}_{S, T, d, V}$ is $R_{S, T, d}$, and the dimensional formula follows. The fiber in (2) may be identified with $\left(P_{S} \times P_{T}^{-}\right) / R_{S, T, d}$, which may be identified with $G_{S}$ via the map

$$
\left(P_{S} \times P_{T}^{-}\right) / R_{S, T, d} \rightarrow G_{S}:\left(p_{S}, p_{T}^{-}\right) \mapsto \gamma_{d}^{-1}\left(\chi_{T}\left(p_{T}^{-}\right)\right)\left(\chi_{S}\left(p_{S}\right)\right)^{-1} .
$$

Remark 2.18. - It follows that the number of orbit types for $G \times G$ in $\mathcal{L}$ is exactly the number of generalized BD-triples for $G$.

LEMMA 2.19. - $R_{S, T, d}$ is connected.

Proof. - The projection map $p: R_{S, T, d} \rightarrow P_{S},\left(p_{S}, p_{T}^{-}\right) \mapsto p_{S}$ is surjective and has fiber $N_{T}^{-} \times Z_{T}$, where $Z_{T}$ is the center of the group $M_{T}$. Clearly $N_{T}^{-}$is connected, and $Z_{T}$ is connected by Proposition 8.1.4 of [4]. Since $P_{S}$ is connected, it follows that $R_{S, T, d}$ is also connected.

\section{6. $\left(B \times B^{-}\right)$-orbits in $\mathcal{L}$}

Let $B$ and $B^{-}$be the Borel subgroups of $G$ with Lie algebras $\mathfrak{b}=\mathfrak{h}+\mathfrak{n}$ and $\mathfrak{b}^{-}=\mathfrak{h}+\mathfrak{n}^{-}$ respectively. By Proposition 2.17, to classify $\left(B \times B^{-}\right)$-orbits in $\mathcal{L}$, it suffices to consider $\left(B \times B^{-}\right)$-orbits in $(G \times G) / R_{S, T, d}$ for any generalized BD-triple $(S, T, d)$. 
Let $W$ be the Weyl group of $\Sigma$. For $S \subset \Gamma$, let $W_{S}$ be the subgroup of $W$ generated by elements in $S$, and let $W^{S}$ be the set of minimal length representatives of elements in the cosets in $W / W_{S}$. Fix a representative $\dot{w}$ in $G$ for each $w \in W$. The following assertion can be proved in the same way as Lemma 1.3 in [31]. It also follows directly from Proposition 8.1 of [24].

Proposition 2.20. - Let $(S, T, d)$ be an generalized BD-triple for $G$. Then every $\left(B \times B^{-}\right)$orbit in $(G \times G) / R_{S, T, d}$ is of the form $\left(B \times B^{-}\right)(\dot{w}, \dot{v}) R_{S, T, d}$ for a unique pair $(w, v) \in$ $W \times W^{T}$.

COROLlary 2.21. - Every $\left(B \times B^{-}\right)$-orbit in $\mathcal{L}$ goes through exactly one point in $\mathcal{L}$ of the form $\operatorname{Ad}_{(\dot{w}, \dot{v})} \mathfrak{l}_{S, T, d, V}$, where $(S, T, d)$ is a generalized BD-triple, $V \in \mathcal{L}_{\text {space }}\left(\mathfrak{z}_{S} \oplus \mathfrak{z}_{T}\right)$, and $(w, v) \in W \times W^{T}$.

Since each $(G \times G)$-orbit in $\mathcal{L}$ has finitely many $\left(B \times B^{-}\right)$-orbits, at least one of them is open.

COROLLARY 2.22. - All $(G \times G)$-orbits in $\mathcal{L}$ are $(G \times G)$-spherical homogeneous spaces.

\subsection{The De Concini-Procesi compactifications $Z_{d}(G)$ of $G$}

In this section, we will consider the closure in $\mathcal{L}$ of some special $(G \times G)$-orbits. Namely, when $S=T=\Gamma$ and $d \in I(\Gamma, \Gamma)$, we have the graph $\mathfrak{l}_{\gamma_{d}}$ of $\gamma_{d}: \mathfrak{g} \rightarrow \mathfrak{g}$ as a point in $\mathcal{L}$ :

$$
\mathfrak{l}_{\gamma_{d}}=\left\{\left(x, \gamma_{d}(x)\right): x \in \mathfrak{g}\right\}
$$

The $(G \times G)$-orbit in $\mathcal{L}$ through $\mathfrak{l}_{\gamma_{d}}$ can be identified with $G$ by the map

$$
I_{d}: G \rightarrow(G \times G) \cdot \mathfrak{l}_{\gamma_{d}}: g \mapsto\left\{\left(x, \gamma_{d} \operatorname{Ad}_{g}(x)\right): x \in \mathfrak{g}\right\} .
$$

The identification $I_{d}$ is $(G \times G)$-equivariant if we equip $G$ with the action of $G \times G$ given by

$$
\left(g_{1}, g_{2}\right) \cdot g=\gamma_{d}^{-1}\left(g_{2}\right) g g_{1}^{-1} .
$$

Since an orbit of an algebraic group on a variety is locally closed (Section 8.3 in [14]), the orbit $(G \times G) \cdot \mathfrak{l}_{\gamma_{d}}$ has the same closure in the Zariski topology and in the classical topology. The closure $\overline{(G \times G) \cdot \mathfrak{l}_{\gamma_{d}}}$, called a De Concini-Procesi compactification of $G$, is a smooth projective variety of dimension $n=\operatorname{dim} G$ (see [5, $\S 6]$ ). We denote this closure by $Z_{d}(G)$.

It is known [5] that $G \times G$ has finitely many orbits in $Z_{d}(G)$ indexed by subsets of $\Gamma$. Indeed, for each $S \subset \Gamma$, let $\mathfrak{l}_{S, d} \in \mathcal{L}$ be given by

$$
\mathfrak{l}_{S, d}=\mathfrak{n}_{S} \oplus \mathfrak{n}_{d(S)}^{-}+\left\{\left(x, \gamma_{d}(x)\right): x \in \mathfrak{m}_{S}\right\} .
$$

Choose $\lambda \in \mathfrak{h}$ such that $\alpha(\lambda)=0$ for $\alpha \in S$ and $\alpha(\lambda)>0$ for $\alpha \in \Gamma-S$, and let $e^{\lambda}: \mathbb{C}^{*} \rightarrow H$ be the one parameter subgroup of $H$ corresponding to $\lambda$. Then it is easy to see that

$$
\lim _{t \rightarrow+\infty} \operatorname{Ad}_{\left(e^{\lambda}(t), e\right)} \mathfrak{l}_{\gamma_{d}}=\mathfrak{l}_{S, d} \in \operatorname{Gr}(n, \mathfrak{g} \oplus \mathfrak{g}) .
$$

Thus $\mathfrak{l}_{S, d} \in Z_{d}(G)$. It is easy to see that $\mathfrak{l}_{\gamma_{d}} \in \mathcal{L}^{\varepsilon}(\Gamma, \Gamma, d)$ for $\varepsilon=\left(\operatorname{dim} \mathfrak{h}-\operatorname{dim} \mathfrak{h}^{\gamma_{d}}\right) \bmod 2$. Thus $\mathfrak{l}_{S, d} \in \mathcal{L}^{\varepsilon}\left(S, d(S),\left.d\right|_{S}\right)$ for the same value of $\varepsilon$.

TheOREM 2.23. - (See [5].) For every $d \in I(\Gamma, \Gamma), Z_{d}(G)=\bigcup_{S \subset \Gamma}(G \times G) \cdot \mathfrak{l}_{S, d}$.

$4^{\mathrm{e}}$ SÉRIE - TOME $39-2006-\mathrm{N}^{\circ} 2$ 


\subsection{Closures of $(G \times G)$-orbits in $\mathcal{L}$}

Let $(S, T, d, V)$ be a quadruple where $(S, T, d)$ is a generalized BD-triple and $V \in \mathcal{L}_{\text {space }}\left(\mathfrak{z}_{S} \oplus\right.$ $\left.\mathfrak{z}_{T}\right)$. For $\mathfrak{l}_{S, T, d, V}$ given in (2.4), we will now study the closure of the $(G \times G)$-orbit through $\mathfrak{l}_{S, T, d, V}$ in $\operatorname{Gr}(n, \mathfrak{g} \oplus \mathfrak{g})$. To this end, let $\operatorname{Gr}\left(m, \mathfrak{g}_{S} \oplus \mathfrak{g}_{T}\right)$ be the Grassmannian of $m$-dimensional subspaces in $\mathfrak{g}_{S} \oplus \mathfrak{g}_{T}$, where $m=\operatorname{dim} \mathfrak{g}_{S}$. For the Lie algebra isomorphism $\gamma_{d}: \mathfrak{g}_{S} \rightarrow \mathfrak{g}_{T}$ given in (2.2), let

$$
\mathfrak{l}_{\gamma_{d}}=\left\{\left(x, \gamma_{d}(x)\right): x \in \mathfrak{g}_{S}\right\} \in \operatorname{Gr}\left(m, \mathfrak{g}_{S} \oplus \mathfrak{g}_{T}\right) .
$$

NotATion 2.24. - Let $Z_{d}\left(G_{S}\right)$ be the closure of $\left(G_{S} \times G_{T}\right) \cdot \mathfrak{l}_{\gamma_{d}}$ in $\operatorname{Gr}\left(m, \mathfrak{g}_{S} \oplus \mathfrak{g}_{T}\right)$. Under the identification

$$
G_{S} \rightarrow\left(G_{S} \times G_{T}\right) \cdot \mathfrak{l}_{\gamma_{d}}: g \mapsto\left\{\left(x, \gamma_{d} \operatorname{Ad}_{g} x\right): x \in \mathfrak{g}_{S}\right\},
$$

$Z_{d}\left(G_{S}\right)$ can be regarded as a $\left(P_{S} \times P_{T}^{-}\right)$-equivariant compactification of $G_{S}$, where $P_{S} \times P_{T}^{-}$ act on $G_{S}$ by

$$
\left(p_{S}, p_{T}^{-}\right) \cdot g_{S}=\gamma_{d}^{-1}\left(\chi_{T}\left(p_{T}^{-}\right)\right) g_{S}\left(\chi_{S}\left(p_{S}\right)\right)^{-1}, \quad\left(p_{S}, p_{T}^{-}\right) \in P_{S} \times P_{T}^{-}, \quad g_{S} \in G_{S}
$$

(see Notation 2.11), and on $\operatorname{Gr}\left(m, \mathfrak{g}_{S} \oplus \mathfrak{g}_{T}\right)$ via the group homomorphism $\chi_{S} \times \chi_{T}: P_{S} \times P_{T}^{-} \rightarrow$ $G_{S} \times G_{T}$.

Proposition 2.25. - For every generalized BD-triple $(S, T, d)$ and every $V \in$ $\mathcal{L}_{\text {space }}\left(\mathfrak{z}_{S} \oplus \mathfrak{z}_{T}\right)$,

(1) the closure $\overline{(G \times G) \cdot \mathfrak{l}_{S, T, d, V}}$ in $\operatorname{Gr}(n, \mathfrak{g} \oplus \mathfrak{g})$ is a smooth subvariety of $\operatorname{Gr}(n, \mathfrak{g} \oplus \mathfrak{g})$ of dimension $n-z$, where $n=\operatorname{dim} \mathfrak{g}$ and $z=\operatorname{dim}_{S}$, and the map

$$
\begin{aligned}
\mathbf{a}:(G \times G) \times_{\left(P_{S} \times P_{T}^{-}\right)} Z_{d}\left(G_{S}\right) & \rightarrow \overline{(G \times G) \cdot \mathfrak{l}_{S, T, d, V}}: \\
{\left[\left(g_{1}, g_{2}\right), \mathfrak{l}\right] } & \mapsto \operatorname{Ad}_{\left(g_{1}, g_{2}\right)}\left(V+\left(\mathfrak{n}_{S} \oplus \mathfrak{n}_{T}^{-}\right)+\mathfrak{l}\right)
\end{aligned}
$$

is a $(G \times G)$-equivariant isomorphism;

(2) $\overline{(G \times G) \cdot \mathfrak{l}_{S, T, d, V}}$ is the finite disjoint union

$$
\overline{(G \times G) \cdot \mathfrak{l}_{S, T, d, V}}=\bigcup_{S_{1} \subset S}(G \times G) \cdot \mathfrak{l}_{S_{1}, d\left(S_{1}\right), d_{1}, V_{1}},
$$

where for $S_{1} \subset S$, we set $d_{1}=\left.d\right|_{S_{1}}$, and $V_{1}=V+\left\{\left(x, \gamma_{d}(x)\right): x \in \mathfrak{h}_{S} \cap \mathfrak{z} S_{1}\right\} \subset \mathfrak{z}_{S_{1}} \oplus \mathfrak{z} T_{1}$.

Proof. - Since $G / P_{S} \times G / P_{T}^{-}$is complete, the image of a is closed. Since $\mathbf{a}\left[(e, e), \mathfrak{l}_{\gamma_{d}}\right]=$ $\mathfrak{l}_{S, T, d, V}$ and $\mathbf{a}$ is $(G \times G)$-equivariant, a has dense image. Hence $\mathbf{a}$ is onto. (2) follows easily from the fact that $\mathbf{a}$ is onto and the description of orbits in $Z_{d}\left(G_{S}\right)$ in Theorem 2.23. Further, by this description of orbits, for $\mathfrak{l} \in Z_{d}\left(G_{S}\right)$, the nilradical of $V+\left(\mathfrak{n}_{S} \oplus \mathfrak{n}_{T}^{-}\right)+\mathfrak{l}$ contains $\mathfrak{n}_{S} \oplus \mathfrak{n}_{T}^{-}$. It follows that if $\mathbf{a}\left[\left(g_{1}, g_{2}\right), \mathfrak{l}\right] \in \mathbf{a}\left[(e, e), Z_{d}\left(G_{S}\right)\right]$, then $\left(g_{1}, g_{2}\right)$ is in the normalizer of the nilradical of $V+\left(\mathfrak{n}_{S} \oplus \mathfrak{n}_{T}^{-}\right)+\mathfrak{l}$. This normalizer, and hence also $\left(g_{1}, g_{2}\right)$, is contained in $P_{S} \times P_{T}^{-}$. Now suppose $\mathbf{a}\left[\left(g_{1}, g_{2}\right), \mathfrak{l}\right]=\mathbf{a}\left[\left(x_{1}, x_{2}\right), \mathfrak{l}_{1}\right]$ for $x_{1}, x_{2} \in G$ and $\mathfrak{l}_{1} \in Z_{d}\left(G_{S}\right)$. Then $\mathbf{a}\left[\left(x_{1}^{-1} g_{1}, x_{2}^{-1} g_{2}\right), \mathfrak{l}\right]=\mathbf{a}\left[(e, e), \mathfrak{l}_{1}\right]$, so by the above $\left(x_{1}^{-1} g_{1}, x_{2}^{-1} g_{2}\right) \in P_{S} \times P_{T}^{-}$. It follows easily that $\mathbf{a}$ is injective.

To show that the differential $\mathbf{a}_{*}$ of $\mathbf{a}$ is injective everywhere, it suffices to show $\mathbf{a}_{*}$ is injective at all points $[(e, e), \mathfrak{l}]$ by $(G \times G)$-equivariance. For $X \in \mathfrak{g} \oplus \mathfrak{g}$, let $\xi_{X}$ be the vector field on $(G \times G) \times{ }_{\left(P_{S} \times P_{T}^{-}\right)} Z_{d}\left(G_{S}\right)$ tangent to the $(G \times G)$-action. Recall that the tangent 
space at a plane $U$ to the $\operatorname{Grassmannian} \operatorname{Gr}(n, Y)$ may be identified with $\operatorname{Hom}(U, Y / U)$. Since $Z_{d}\left(G_{S}\right) \subset \operatorname{Gr}\left(m, \mathfrak{g}_{S}+\mathfrak{g}_{T}\right)$, it follows that the tangent space to $(G \times G) \times_{\left(P_{S} \times P_{T}^{-}\right)} Z_{d}\left(G_{S}\right)$ at $[(e, e), \mathfrak{l}]$ is spanned by the set of $\xi_{X}$ for $X \in \mathfrak{n}_{S}^{-} \oplus \mathfrak{n}_{T}$ and the set of $\phi$ in $\operatorname{Hom}\left(\mathfrak{l},\left(\mathfrak{g}_{S} \oplus \mathfrak{g}_{T}\right) / \mathfrak{l}\right)$ tangent to $Z_{d}\left(G_{S}\right)$. We identify the tangent space to $\operatorname{Gr}(n, \mathfrak{g} \oplus \mathfrak{g})$ at a $[(e, e), \mathfrak{l}]$ with $\operatorname{Hom}(V+$ $\left.\mathfrak{n}_{S} \oplus \mathfrak{n}_{T}^{-}+\mathfrak{l},(\mathfrak{g} \oplus \mathfrak{g}) /\left(V+\mathfrak{n}_{S} \oplus \mathfrak{n}_{T}^{-}+\mathfrak{l}\right)\right)$. Then $\mathbf{a}_{*}\left(\xi_{X}\right)=\eta_{X}$, where for $Y \in V+\mathfrak{n}_{S} \oplus \mathfrak{n}_{T}^{-}+\mathfrak{l}$, $\eta_{X}(Y)=\operatorname{ad}_{X}(Y) \bmod \left(V+\mathfrak{n}_{S} \oplus \mathfrak{n}_{T}^{-}+\mathfrak{l}\right)$. Moreover, $\mathbf{a}_{*}(\phi)=\tilde{\phi}$, where $\tilde{\phi}(x)=\phi(x)$ for $x \in \mathfrak{l}$, and $\tilde{\phi}(y)=0$ if $y \in V+\mathfrak{n}_{S} \oplus \mathfrak{n}_{T}^{-}$. We further identify $(\mathfrak{g} \oplus \mathfrak{g}) /\left(V+\mathfrak{n}_{S} \oplus \mathfrak{n}_{T}^{-}+\mathfrak{l}\right)$ with $\left(\mathfrak{z}_{S} \oplus \mathfrak{z}_{T}\right) / V+\mathfrak{n}_{S}^{-} \oplus \mathfrak{n}_{T}+\left(\mathfrak{g}_{S} \oplus \mathfrak{g}_{T}\right) /$ l. Now suppose that $\mathbf{a}_{*}\left(\xi_{X}+\phi\right)=0$. Since $\operatorname{ad}_{X}(\mathfrak{l}) \subset \mathfrak{n}_{S}^{-} \oplus \mathfrak{n}_{T}$ and $\tilde{\phi}(\mathfrak{l}) \subset\left(\mathfrak{g}_{S} \oplus \mathfrak{g}_{T}\right) / \mathfrak{l}$, it follows that $\tilde{\phi}=0$. Hence, $\phi=0$, and $\xi_{X}=0$ since $\mathbf{a}_{*}$ is injective on $\mathfrak{n}_{S}^{-} \oplus \mathfrak{n}_{T}$. By Corollary 14.10 of [12], $\mathbf{a}$ is an isomorphism.

Consider now the case when $S$ and $T$ are the empty set $\emptyset$, so $d=1$. By Theorem 2.16, every $(G \times G)$-orbit in $\mathcal{L}^{0}(\emptyset, \emptyset, 1) \cup \mathcal{L}^{1}(\emptyset, \emptyset, 1)$ goes through a unique Lagrangian subalgebra of the form

$$
\mathfrak{l}_{V}=V+\left(\mathfrak{n} \oplus \mathfrak{n}^{-}\right),
$$

where $V \in \mathcal{L}_{\text {space }}(\mathfrak{h} \oplus \mathfrak{h})$. The following fact follows immediately from Proposition 2.25.

COROLlaRY 2.26. - For every $V \in \mathcal{L}_{\text {space }}(\mathfrak{h} \oplus \mathfrak{h})$, the $(G \times G)$-orbit through $\mathfrak{l}_{V}$ is isomorphic to $G / B \times G / B^{-}$. These are the only closed $(G \times G)$-orbits in $\mathcal{L}$.

\section{Corollary 2.27. - $\mathcal{L}$ has two connected components.}

Proof. - In Section 2.1, we observed that $\mathcal{L}$ has at least two connected components, namely $\mathcal{L}^{0}$ and $\mathcal{L}^{1}$. Since every orbit of an algebraic group on a variety has a closed orbit in its boundary (see Section 8.3 in [14]), every point in $\mathcal{L}$ is in the same connected component as $\mathfrak{l}_{V}$ for some $V \in \mathcal{L}_{\text {space }}(\mathfrak{h} \oplus \mathfrak{h})$. Thus $\mathcal{L}$ has at most two connected components.

\subsection{The geometry of the strata $\mathcal{L}^{\varepsilon}(S, T, d)$}

Fix a generalized BD-triple $(S, T, d)$ and an $\varepsilon \in\{0,1\}$. Let $P_{S} \times P_{T}^{-}$act on $G_{S}$ by (2.12) and trivially on $\mathcal{L}_{\text {space }}^{\varepsilon}\left(\mathfrak{z}_{S} \oplus \mathfrak{z}_{T}\right)$. Consider the associated bundle $(G \times G) \times_{\left(P_{S} \times P_{T}^{-}\right)}$ $\left(G_{S} \times \mathcal{L}_{\text {space }}^{\varepsilon}\left(\mathfrak{z}_{S} \oplus \mathfrak{z}_{T}\right)\right)$ over $G / P_{S} \times G / P_{T}^{-}$and the map

$$
\begin{aligned}
\mathbf{a}:(G \times G) \times_{\left(P_{S} \times P_{T}^{-}\right)}\left(G_{S} \times \mathcal{L}_{\text {space }}^{\varepsilon}\left(\mathfrak{z}_{S} \oplus \mathfrak{z}_{T}\right)\right) & \rightarrow \mathcal{L}^{\varepsilon}(S, T, d), \\
{\left[\left(g_{1}, g_{2}\right),(g, V)\right] } & \mapsto \operatorname{Ad}_{\left(g_{1}, g_{2}\right)} \mathfrak{l}_{g, V},
\end{aligned}
$$

where $\mathfrak{l}_{g, V}=V+\left(\mathfrak{n}_{S} \oplus \mathfrak{n}_{T}^{-}\right)+\left\{\left(x, \gamma_{d} \operatorname{Ad}_{g}(x)\right): x \in \mathfrak{g}_{S}\right\}$ for $g \in G_{S}$.

Proposition 2.28. - For every $S, T \subset \Gamma, d \in I(S, T)$, and $\varepsilon \in\{0,1\}, \mathcal{L}^{\varepsilon}(S, T, d)$ is a smooth connected subvariety of $\operatorname{Gr}(n, \mathfrak{g} \oplus \mathfrak{g})$ of dimension $n+\frac{z(z-3)}{2}$, where $n=\operatorname{dim} \mathfrak{g}$ and $z=\operatorname{dim}_{S}$, and the map a in (2.14) is a $(G \times G)$-equivariant isomorphism.

Proof. - Consider the $(G \times G)$-equivariant projection

$$
J: \mathcal{L}^{\varepsilon}(S, T, d) \rightarrow G / P_{S} \times G / P_{T}^{-}: \mathfrak{l}\left(\mathfrak{p}, \mathfrak{p}^{\prime}, \mu, V\right) \mapsto\left(\mathfrak{p}, \mathfrak{p}^{\prime}\right) .
$$

Let $\mathcal{F}^{\varepsilon}(S, T, d)$ be the fibre of $J$ over the point $\left(\mathfrak{p}_{S}, \mathfrak{p}_{T}^{-}\right) \in G / P_{S} \times G / P_{T}^{-}$. By Lemma 4, p. 26 of [29], the map

$$
(G \times G) \times_{\left(P_{S} \times P_{T}^{-}\right)} \mathcal{F}^{\varepsilon}(S, T, d) \rightarrow \mathcal{L}^{\varepsilon}(S, T, d):\left[\left(g_{1}, g_{2}\right), \mathfrak{l}\right] \mapsto \operatorname{Ad}_{\left(g_{1}, g_{2}\right)} \mathfrak{l}
$$

$4^{\text {e }}$ SÉRIE - TOME $39-2006-\mathrm{N}^{\circ} 2$ 
is a $(G \times G)$-equivariant isomorphism. By Lemma 2.6,

$$
\mathcal{F}^{\varepsilon}(S, T, d)=\left\{\mathfrak{l}_{g, V}: g \in G_{S}, V \in \mathcal{L}_{\text {space }}^{\varepsilon}\left(\mathfrak{z}_{S} \oplus \mathfrak{z}_{T}\right)\right\} .
$$

The identification $G_{S} \times \mathcal{L}_{\text {space }}^{\varepsilon}\left(\mathfrak{z}_{S} \oplus \mathfrak{z}_{T}\right) \rightarrow \mathcal{F}^{\varepsilon}(S, T, d):(g, V) \mapsto \mathfrak{l}_{g, V}$ is $\left(P_{S} \times P_{T}^{-}\right)$equivariant. It follows that a is a $(G \times G)$-equivariant isomorphism. The dimension claim follows from Propositions 2.2 and 2.17. Smoothness and connectedness of $\mathcal{L}^{\varepsilon}(S, T, d)$ follow easily.

\subsection{The geometry of the closure of $\overline{\mathcal{L}^{\varepsilon}(S, T, d)}$}

For a generalized BD-triple $(S, T, d)$ and an $\varepsilon \in\{0,1\}$, recall that $Z_{d}\left(G_{S}\right)$ is an $\left(P_{S} \times P_{T}^{-}\right)$equivariant compactification of $G_{S}$. Let again $P_{S} \times P_{T}^{-}$act trivially on $\mathcal{L}_{\text {space }}^{\varepsilon}\left(\mathfrak{z}_{S} \oplus \mathfrak{z}_{T}\right)$. We omit the proof of the following theorem since it is similar to the proof of Theorem 2.25.

THEOREM 2.29. - For every generalized BD-triple $(S, T, d)$ and $\varepsilon \in\{0,1\}$, the closure $\overline{\mathcal{L}^{\varepsilon}(S, T, d)}$ is a smooth algebraic variety of dimension $n+\frac{z(z-3)}{2}$, where $n=\operatorname{dim}(\mathfrak{g}), z=$ $\operatorname{dim} \mathfrak{z}_{S}$, and

$$
\mathbf{a}:(G \times G) \times_{\left(P_{S} \times P_{T}^{-}\right)}\left(Z_{d}\left(G_{S}\right) \times \mathcal{L}_{\text {space }}^{\varepsilon}\left(\mathfrak{z}_{S} \oplus \mathfrak{z}_{T}\right)\right) \rightarrow \overline{\mathcal{L}^{\varepsilon}(S, T, d)},
$$

$$
\left[\left(g_{1}, g_{2}\right),(\mathfrak{l}, V)\right] \mapsto \operatorname{Ad}_{\left(g_{1}, g_{2}\right)}\left(V+\left(\mathfrak{n}_{S} \oplus \mathfrak{n}_{T}^{-}\right)+\mathfrak{l}\right)
$$

is a $(G \times G)$-equivariant isomorphism.

COROLlary 2.30. - For every generalized BD-triple $(S, T, d)$ and $\varepsilon \in\{0,1\}$,

$$
\overline{\mathcal{L}^{\varepsilon}(S, T, d)}=\bigcup_{V \in \mathcal{L}_{\text {space }}^{\varepsilon}(\mathfrak{z} S \oplus \mathfrak{z} T)} \bigcup_{S_{1} \subset S}(G \times G) \cdot \mathfrak{l}_{S_{1}, d\left(S_{1}\right), d, V_{1}\left(V, S_{1}\right)}
$$

is a disjoint union, where for $S_{1} \subset S$ and $V \in \mathcal{L}_{\text {space }}^{\varepsilon}\left(\mathfrak{z}_{S} \oplus \mathfrak{z}_{T}\right)$,

$$
V_{1}\left(V, S_{1}\right)=V+\left\{\left(x, \gamma_{d}(x)\right): x \in \mathfrak{h}_{S} \cap \mathfrak{z}_{S_{1}}\right\} \subset \mathfrak{z}_{S_{1}} \oplus \mathfrak{z} d\left(S_{1}\right) .
$$

Remark 2.31. - (1) Since $Z_{d}\left(G_{S}\right)$ is also the closure in the classical topology of $\left(G_{S} \times G_{T}\right) \cdot \mathfrak{l}_{\gamma_{d}}$ inside $\operatorname{Gr}\left(m, \mathfrak{g}_{S} \oplus \mathfrak{g}_{T}\right), \mathcal{L}^{\varepsilon}(S, T, d)$ also has the same closure in the two topologies of $\operatorname{Gr}(n, \mathfrak{g} \oplus \mathfrak{g})$.

(2) Since $Z_{d}\left(G_{S}\right)-G_{S}$ has dimension strictly lower than $m=\operatorname{dim} G_{S}$, it follows from the proof of Theorem 2.29 that $\overline{\mathcal{L}^{\varepsilon}(S, T, d)}-\mathcal{L}^{\varepsilon}(S, T, d)$ is of strictly lower dimension than $\overline{\mathcal{L}^{\varepsilon}(S, T, d)}$.

\subsection{Irreducible components of $\mathcal{L}$}

Since each $\overline{\mathcal{L}^{\varepsilon}(S, T, d)}$ is smooth and connected, it is a closed irreducible subvariety of $\mathcal{L}$. Since

$$
\mathcal{L}=\bigcup_{\varepsilon \in\{0,1\}} \bigcup_{S, T \subset \Gamma, d \in I(S, T)} \overline{\mathcal{L}^{\varepsilon}(S, T, d)}
$$

is a finite union, the irreducible components of $\mathcal{L}$ are those $\overline{\mathcal{L}^{\varepsilon}(S, T, d)}$ that are not properly contained in some other such set.

THEOREM 2.32. $-\overline{\mathcal{L}^{\varepsilon}(S, T, d)}$ is an irreducible component of $\mathcal{L}$ unless $|\Gamma-S|=1$, $T=d_{1}(S)$ for some $d_{1} \in I(\Gamma, \Gamma), d=\left.d_{1}\right|_{S}$, and $\varepsilon=\left(\operatorname{dim} \mathfrak{h}-\operatorname{dim} \mathfrak{h}^{\gamma_{d_{1}}}\right) \bmod 2$. 
Proof. - When $(S, T, d, \varepsilon)$ are as described in the proposition, $\operatorname{dim}_{S}=1$, so $\mathcal{L}^{\varepsilon}(S, T, d)$ consists of a single $(G \times G)$-orbit which lies in $Z_{d_{1}}(G)$ by Theorem 2.23. We need to show that this is the only non-trivial case when the closure $\overline{\mathcal{L}^{\varepsilon}(S, T, d)}$ is contained in another $\overline{\mathcal{L}^{\varepsilon}\left(S_{1}, T_{1}, d_{1}\right)}$.

Assume that $\mathcal{L}^{\varepsilon}(S, T, d)$ is in the boundary of $\overline{\mathcal{L}^{\varepsilon}\left(S_{1}, T_{1}, d_{1}\right)}$. Then by Corollary $2.30, S \subset S_{1}$ and $T \subset T_{1}$. By Remark 2.31, $\operatorname{dim} \mathcal{L}^{\varepsilon}(S, T, d)<\operatorname{dim} \mathcal{L}^{\varepsilon}\left(S_{1}, T_{1}, d_{1}\right)$, and thus

$$
\frac{1}{2} \operatorname{dim}\left(\mathfrak{z}_{S}\right)\left(\operatorname{dim}\left(\mathfrak{z}_{S}\right)-3\right)<\frac{1}{2} \operatorname{dim}\left(\mathfrak{z}_{S_{1}}\right)\left(\operatorname{dim}\left(\mathfrak{z}_{S_{1}}\right)-3\right)
$$

by the dimension formula in Proposition 2.28. Since $S \subset S_{1}$, so $\operatorname{dim}\left(\mathfrak{z}_{S}\right) \geqslant \operatorname{dim}\left(\mathfrak{z}_{S_{1}}\right)$, these two inequalities imply that $\operatorname{dim}\left(\mathfrak{z}_{S_{1}}\right)=0$ and $\operatorname{dim}\left(\mathfrak{z}_{S}\right)=1$ or 2 . In particular, $S_{1}=T_{1}=\Gamma$, so $\varepsilon=\left(\operatorname{dim} \mathfrak{h}-\operatorname{dim} \mathfrak{h}^{\gamma_{d_{1}}}\right) \bmod 2$, and $\overline{\mathcal{L}^{\varepsilon}\left(S_{1}, T_{1}, d_{1}\right)}=Z_{d_{1}}(G)$.

If $\operatorname{dim}\left(\mathfrak{z}_{S}\right)=2, \mathcal{L}^{\varepsilon}(S, T, d)$ contains infinitely many $(G \times G)$-orbits by Theorem 2.16 and Proposition 2.2. Since $Z_{d_{1}}(G)$ has only finitely many $(G \times G)$-orbits, $\mathcal{L}^{\varepsilon}(S, T, d)$ cannot be contained in $Z_{d_{1}}(G)$. Assume that $\operatorname{dim}\left(\mathfrak{z}_{S}\right)=1$. Then by Proposition $2.28, \mathcal{L}^{\varepsilon}(S, T, d)$ is a single $(G \times G)$-orbit. By the description of the $(G \times G)$-orbits in $Z_{d_{1}}(G)$ in Theorem 2.23, $T$ and $d$ must be as described in the proposition.

Example 2.33. - For $\mathfrak{g}=(2, \mathbb{C}), \mathcal{L}$ has two irreducible components. One is the De ConciniProcesi compactification $Z_{\text {id }}(G)$ of $G=P S L(2, C)$ which is isomorphic to $\mathbb{C} P^{3}$ (see [5]), and the other is the closed $(G \times G)$-orbit through $\mathfrak{h}_{\Delta}+\left(\mathfrak{n} \oplus \mathfrak{n}^{-}\right)$, and is isomorphic to $\mathbb{C} P^{1} \times \mathbb{C} P^{1}$.

For $\mathfrak{g}=(3, \mathbb{C})$, there are four irreducible components $Z_{\mathrm{id}}(G), Z_{d_{1}}(G), C_{1}$ and $C_{2}$, where $Z_{\text {id }}(G)$ and $Z_{d_{1}}(G)$ are the two De Concini-Procesi compactifications of $G=P S L(3, \mathbb{C})$ corresponding to the identity and the non-trivial automorphism of the Dynkin diagram of $(3, \mathbb{C})$, and $C_{1}$ and $C_{2}$ are the two components $\mathcal{L}_{0}(\emptyset, \emptyset, d)$ and $\mathcal{L}_{1}(\emptyset, \emptyset, d)$. Both $C_{1}$ and $C_{2}$ have dimension 7. Moreover, $Z_{\text {id }}(G) \cap C_{1}$ is a 6-dimensional closed $(G \times G)$-orbit, and so is $Z_{d_{1}}(G) \cap C_{2}$.

\section{Classification of $G_{\Delta}$-orbits in $\mathcal{L}$}

By Theorem 2.16, to describe the $G_{\Delta}$-orbits in $\mathcal{L}$, it suffices to describe $\left(G_{\Delta}, R_{S, T, d}\right)$-double cosets in $G \times G$ for all generalized BD-triples $(S, T, d)$ for $\Gamma$, where $R_{S, T, d}$ is given by (2.6). A general double coset theorem in [24] classifies $\left(R_{S^{\prime}, T^{\prime}, d^{\prime}}, R_{S, T, d}\right)$-double cosets in $G \times G$ for two arbitrary generalized BD-triples $\left(S^{\prime}, T^{\prime}, d^{\prime}\right)$ and $(S, T, d)$. However, the proof in [24] is rather technically involved. In this section, we present a simplified proof for the special case when $R_{S^{\prime}, T^{\prime}, d^{\prime}}=G_{\Delta}$. Our method and the one used in [24] are both adapted from [33]. The $G_{\Delta^{-}}$ orbits in the wonderful compactifications were also studied by Lusztig in [26] and [27] using a somewhat different method.

\subsection{Some results on Weyl groups and generalized BD-triples}

The results in this section, while different in presentation, are closely related to some combinatorial results in [2], which were used and extended in [26] and [27]. In particular, the limit of a sequence studied in [2] is closely related to the set $S(v, d)$ in Definition 3.2.

Notation 3.1. - Let $W$ be the Weyl group of $\Gamma$. For $F \subset \Gamma$, let $W_{F}$ be the subgroup of $W$ generated by elements in $F$. If $E, F \subset \Gamma$, let ${ }^{E} W^{F}$ be the set of minimal length representatives for double cosets from $W_{E} \backslash W / W_{F}$, and set $W^{F}={ }^{\emptyset} W^{F}$. If $E_{1}, E_{2} \subset F$, the set of minimal length representatives in $W_{F}$ for the double cosets from $W_{E_{1}} \backslash W_{F} / W_{E_{2}}$ will be denoted by

$4^{\text {e }}$ SÉRIE - TOME $39-2006-\mathrm{N}^{\circ} 2$ 
$E_{1}\left(W_{F}\right)^{E_{2}}$. If $u \in E^{E_{1}}\left(W_{F}\right)^{E_{2}}$ and $v \in E_{1}^{\prime}\left(W_{F^{\prime}}\right)^{E_{2}^{\prime}}$, we can regard both $u \in W_{F}$ and $v \in W_{F^{\prime}}$ as elements in $W$, and by $u v$ we will mean their product in $W$.

Definition 3.2. - Let $(S, T, d)$ be a generalized BD-triple in $\Gamma$. For $v \in W^{T}$, regarding $v d$ as a map $S \rightarrow \Delta$, we define $S(v, d) \subset S$ to be the largest subset in $S$ that is invariant under $v d$. In other words,

$$
S(v, d)=\left\{\alpha \in S:(v d)^{n} \alpha \in S, \forall \text { integer } n \geqslant 1\right\} .
$$

Parts (1) and (2) in the following Lemma 3.3 follow directly from Proposition 2.7.5 of [4] or Lemma 4.3 of [33], and part (3) is a special case of Lemma 5.3 in [24].

LEMMA 3.3.-

(1) If $w \in{ }^{S} W^{T}$ and $u \in\left(W_{S}\right)^{S \cap w(T)}$, then $u w \in W^{T}$;

(2) Every $v \in W^{T}$ has a unique decomposition $v=u w$, where $w \in{ }^{S} W^{T}$ and $u \in$ $\left(W_{S}\right)^{S \cap w(T)}$. Moreover, $l(v)=l(u)+l(w)$;

(3) For $w \in{ }^{S} W^{T}$, set $T_{w}=S \cap w(T)$ and $S_{w}=d^{-1}\left(T \cap w^{-1}(S)\right)$ and regard $\left(S_{w}, T_{w}, w d\right)$ as a generalized $B D$-triple in $S$. Then for any $u \in\left(W_{S}\right)^{T_{w}}$, one has $S_{w}(u, w d)=$ $S(u w, d)$, where $S_{w}(u, w d)$ is the largest subset of $S_{w}$ that is invariant under $u w d$.

Notation 3.4. - Fix a generalized BD-triple $(S, T, d)$. Let $\mathcal{Q}_{S, T, d}$ denote the set of all sequences $\mathbf{q}=\left\{\mathbf{q}_{i}\right\}_{i \geqslant 0}$ of quadruples

$$
\mathbf{q}_{i}=\left(S_{i}, T_{i}, d_{i}, w_{i}\right), \quad i \geqslant 0,
$$

where, for each $i \geqslant 0$,

(1) $\left(S_{i}, T_{i}, d_{i}\right)$ is a generalized BD-triple and $\left(S_{0}, T_{0}, d_{0}\right)=(S, T, d)$;

(2) $w_{i} \in S_{i}\left(W_{S_{i-1}}\right)^{T_{i}}$ (we set $S_{-1}=\Gamma$ );

(3) the triple $\left(S_{i+1}, T_{i+1}, d_{i+1}\right)$ is obtained from $\mathbf{q}_{i}$ as follows:

$$
T_{i+1}=S_{i} \cap w_{i}\left(T_{i}\right), \quad d_{i+1}=w_{i} d_{i}, \quad S_{i+1}=d_{i+1}^{-1}\left(T_{i+1}\right) .
$$

Note that $S_{i+1}, T_{i+1} \subset S_{i}$ for all $i$. For $\mathbf{q}=\left\{\mathbf{q}_{i}\right\}_{i \geqslant 0} \in \mathcal{Q}_{S, T, d}$, let $i_{0}$ be the smallest integer such that $S_{i_{0}+1}=S_{i_{0}}$. Then it is easy to see that

$$
\mathbf{q}_{i}=\mathbf{q}_{i_{0}+1}=\left(S_{i_{0}}, w_{i_{0}}\left(T_{i_{0}}\right), w_{i_{0}} d_{i_{0}}, 1\right)
$$

for all $i \geqslant i_{0}+1$, where 1 is the identity element of $W$. Set

$$
v_{\infty}(\mathbf{q})=w_{i_{0}} w_{i_{0}-1} \cdots w_{0} \quad \text { and } \quad S_{\infty}(\mathbf{q})=S_{i_{0}}
$$

Proposition 3.5 is a direct consequence of Lemma 3.3. See also Proposition 2.5 of [26].

Proposition 3.5. - Let $(S, T, d)$ be a generalized BD-triple for $\Gamma$. Then for any $\mathbf{q} \in \mathcal{Q}_{S, T, d}$, $v:=v_{\infty}(\mathbf{q}) \in W^{T}$, and $S_{\infty}(\mathbf{q})=S(v, d)$. Moreover, the map $\mathcal{Q}_{S, T, d} \rightarrow W^{T}: \mathbf{q} \mapsto v_{\infty}(\mathbf{q})$ is bijective.

\subsection{A double coset theorem}

For this section, $G$ will be a connected complex reductive Lie group with Lie algebra $\mathfrak{g}$, not necessarily of adjoint type. We use the same notation as in Notations 2.11 and 3.1 for various subalgebras of $\mathfrak{g}$ and subgroups of $G$. We will define a class of subgroups $R$ of $G \times G$ that are slightly more general than the groups $R_{S, T, d}$, and we will prove a theorem on $\left(G_{\Delta}, R\right)$-double cosets in $G \times G$ for such an $R$. 
Definition 3.6. - Let $(S, T, d)$ be a generalized BD-triple in $\Gamma$. Let $C_{S}$ (respectively $\left.C_{T}\right)$ be a subgroup of the center $Z_{S}$ (respectively $Z_{T}$ ) of $M_{S}$ (respectively $M_{T}$ ), and let $\theta_{d}: M_{S} / C_{S} \rightarrow$ $M_{T} / C_{T}$ be a group isomorphism that maps the one-dimensional unipotent subgroup of $M_{S} / C_{S}$ defined by $\alpha$ to the corresponding subgroup of $M_{T} / C_{T}$ defined by $d \alpha$ for each $\alpha \in[S]$. By a $(S, T, d)$-admissible subgroup of $G \times G$ we mean a subgroup $R=R\left(C_{S}, C_{T}, \theta_{d}\right)$ of $P_{S} \times P_{T}^{-}$ of the form

$$
R\left(C_{S}, C_{T}, \theta_{d}\right)=\left\{\left(m, m^{\prime}\right) \in M_{S} \times M_{T}: \theta_{d}\left(m C_{S}\right)=m^{\prime} C_{T}\right\}\left(N_{S} \times N_{T}^{-}\right) .
$$

Clearly $R\left(Z_{S}, Z_{T}, \gamma_{d}\right)=R_{S, T, d}$. Let $R$ be any $(S, T, d)$-admissible subgroup of $G \times G$. Recall that the subset $S(v, d)$ of $S$ for $v \in W^{T}$ is defined in (3.1). If $\dot{v}$ is a representative of $v$ in $G$, set

$$
R_{\dot{v}}=\left(M_{S(v, d)} \times M_{S(v, d)}\right) \cap\left(\left(\mathrm{id} \times \operatorname{Ad}_{\dot{v}}\right) R\right),
$$

where $\operatorname{Ad}_{\dot{v}}: G \rightarrow G: g \mapsto \dot{v} g \dot{v}^{-1}$. Let $R_{\dot{v}}$ act on $M_{S(v, d)}$ (from the right) by

$$
m \cdot\left(m_{1}, m_{1}^{\prime}\right)=\left(m_{1}^{\prime}\right)^{-1} m m_{1}, \quad m \in M_{S(v, d)},\left(m_{1}, m_{1}^{\prime}\right) \in R_{\dot{v}} .
$$

For $\left(g_{1}, g_{2}\right) \in G \times G$, let $\left[g_{1}, g_{2}\right]$ be the double coset $G_{\Delta}\left(g_{1}, g_{2}\right) R$ in $G \times G$.

THEOREM 3.7. - Let $(S, T, d)$ be a generalized BD-triple, and let $R=R\left(C_{S}, C_{T}, \theta_{d}\right)$ be an $(S, T, d)$-admissible subgroup of $G \times G$ as given in (3.2). For $v \in W^{T}$, let $S(v, d) \subset S$ be given in (3.1), and let $\dot{v}$ be a fixed representative of $v$ in $G$. Then

(1) every $\left(G_{\Delta}, R\right)$-double coset in $G \times G$ is of the form $[m, \dot{v}]$ for some $v \in W^{T}$ and $m \in M_{S(v, d)}$

(2) Two double cosets $\left[m_{1}, \dot{v}_{1}\right]$ and $\left[m_{2}, \dot{v}_{2}\right]$ in (1) coincide if and only if $v_{1}=v_{2}=v$ and $m_{1}$ and $m_{2}$ are in the same $R_{\dot{v}}$-orbit in $M_{S(v, d)}$ for the $R_{\dot{v}}$ action on $M_{S(v, d)}$ given in (3.3).

We present the main induction step in the proof of Theorem 3.7 in a lemma. Recall that each $w \in{ }^{S} W^{T}$ gives rise to the generalized BD-triple $\left(S_{w}, T_{w}, w d\right)$ in $S$ as in Lemma 3.3. Set

$$
N_{S_{w}}^{S}=N_{S_{w}} \cap M_{S}, \quad \text { and } \quad N_{T_{w}}^{S,-}=N_{T_{w}}^{-} \cap M_{S} .
$$

Fix a representative $\dot{w}$ in $G$, and define

$$
R_{\dot{w}}^{S}=\left(\left(M_{S_{w}} \times M_{T_{w}}\right) \cap\left(\left(\mathrm{id} \times \operatorname{Ad}_{\dot{w}}\right) R\right)\right)\left(N_{S_{w}}^{S} \times N_{T_{w}}^{S,-}\right) .
$$

Then $R_{\dot{w}}^{S}$ is an $\left(S_{w}, T_{w}, w d\right)$-admissible subgroup of $M_{S} \times M_{S}$ defined by the subgroup $C_{S}$ of $Z_{S_{w}}$, the subgroup $w\left(C_{T}\right)$ of $Z_{T_{w}}$ and the group isomorphism $\operatorname{Ad}_{\dot{w}} \theta_{d}: M_{S_{w}} / C_{S} \rightarrow$ $M_{T_{w}} / w\left(C_{T}\right)$.

LEMMA 3.8.-

(1) Every $\left(G_{\Delta}, R\right)$-double coset in $(G \times G)$ is of the form $\left[m, m^{\prime} \dot{w}\right]$ for a unique $w \in{ }^{S} W^{T}$ and some $m \in M_{S}$.

(2) $\left[m_{1}, m_{1}^{\prime} \dot{w}\right]=\left[m_{2}, m_{2}^{\prime} \dot{w}\right]$, where $w \in{ }^{S} W^{T}$ and $\left(m_{1}, m_{1}^{\prime}\right),\left(m_{2}, m_{2}^{\prime}\right) \in M_{S} \times M_{S}$, if and only if $\left(m_{1}, m_{1}^{\prime}\right)$ and $\left(m_{2}, m_{2}^{\prime}\right)$ are in the same $\left(\left(M_{S}\right)_{\Delta}, R_{\dot{w}}^{S}\right)$-double coset in $M_{S} \times M_{S}$.

Proof. - Consider the right action of $P_{S} \times P_{T}^{-}$on $G_{\Delta} \backslash(G \times G)$ by right translations. By the Bruhat decomposition $G=\bigcup_{w \in S_{W} T} P_{S} w P_{T}^{-}$, the set of $\left(P_{S} \times P_{T}^{-}\right)$-orbits is parameterized by the set $\left\{G_{\Delta}(e, \dot{w}): w \in{ }^{S} W^{T}\right\}$. Let $w \in{ }^{S} W^{T}$. The stabilizer subgroup of $P_{S} \times P_{T}^{-}$at $G_{\Delta}(e, \dot{w})$

$4^{\text {e }}$ SÉRIE - TOME $39-2006-\mathrm{N}^{\circ} 2$ 
is $P_{S} \cap \dot{w} P_{T}^{-} \dot{w}^{-1}$ considered as a subgroup of $P_{S} \times P_{T}^{-}$via the embedding

$$
P_{S} \cap \dot{w} P_{T}^{-} \dot{w}^{-1} \rightarrow P_{S} \times P_{T}^{-}: p_{S} \mapsto\left(p_{S}, \dot{w}^{-1} p_{S} \dot{w}\right) .
$$

Thus the set of $R$-orbits in $G_{\Delta} \backslash(G \times G)$ can be identified with the disjoint union over $w \in{ }^{S} W^{T}$ of the spaces of $R$-orbits in $\left(P_{S} \cap \dot{w} P_{T}^{-} \dot{w}^{-1}\right) \backslash\left(P_{S} \times P_{T}^{-}\right)$. Thus, for every $w \in{ }^{S} W^{T}$, we have an injective map

$$
\left(P_{S} \cap \dot{w} P_{T}^{-} \dot{w}^{-1}\right) \backslash P_{S} \times P_{T}^{-} / R \rightarrow G_{\Delta} \backslash G \times G / R
$$

given by $\left(P_{S} \cap \dot{w} P_{T}^{-} \dot{w}^{-1}\right)\left(p_{S}, p_{T}^{-}\right) R \rightarrow\left[p_{S}, \dot{w} p_{T}^{-}\right]$. We will complete the proof by identifying

$$
\left(P_{S} \cap \dot{w} P_{T}^{-} \dot{w}^{-1}\right) \backslash P_{S} \times P_{T}^{-} / R \cong\left(M_{S}\right)_{\Delta} \backslash M_{S} \times M_{S} / R_{\dot{w}}^{S}
$$

through a series of steps. Let $\pi_{S}: P_{S} \rightarrow M_{S}$ and $\pi_{T}: P_{T}^{-} \rightarrow M_{T}$ be the projections with respect to the decompositions $P_{S}=M_{S} N_{S}$ and $P_{T}^{-}=M_{T} N_{T}^{-}$. Then $\pi_{S} \times \pi_{T}: P_{S} \times P_{T}^{-} \rightarrow M_{S} \times M_{T}$ gives an identification

$$
\left(P_{S} \cap \dot{w} P_{T}^{-} \dot{w}^{-1}\right) \backslash P_{S} \times P_{T}^{-} / R \rightarrow R_{1} \backslash M_{S} \times M_{T} / R_{2},
$$

where $R_{1}=\left(\pi_{S} \times \pi_{T}\right)\left(P_{S} \cap \dot{w} P_{T}^{-} \dot{w}^{-1}\right)$ and $R_{2}=\left(M_{S} \times M_{T}\right) \cap R$. Since the projection from $\left(M_{S} \times M_{T}\right) \cap R$ to $M_{T}$ is onto with kernel $\left(C_{S} \times\{e\}\right)$, the map

$$
\phi_{w}:\left(M_{S} \times M_{T}\right) / R_{2} \rightarrow\left(M_{S} \times M_{S}\right) /\left(M_{S}\right)_{\Delta}\left(C_{S} \times\{e\}\right)
$$

that maps $\left(m_{S}, m_{T}\right) R_{2}$ to $\left(m_{S}^{\prime}, m_{S}\right)\left(\left(M_{S}\right)_{\Delta}\left(C_{S} \times\{e\}\right)\right)$ is a well-defined bijection, where for $m_{T} \in M_{T}, m_{S}^{\prime}$ is any element in $M_{S}$ such that $\left(m_{S}^{\prime}, m_{T}\right) \in R_{2}$. Thus $\phi_{w}$ induces an identification

$$
\psi_{w}: R_{1} \backslash M_{S} \times M_{T} / R_{2} \rightarrow R_{3} \backslash M_{S} \times M_{S} /\left(\left(M_{S}\right)_{\Delta}\left(C_{S} \times\{e\}\right)\right),
$$

where

$$
R_{3} \stackrel{\text { def }}{=}\left\{\left(m_{S}^{\prime}, m_{S}\right) \in M_{S} \times M_{S}: \exists m_{T} \in M_{T} \text { such that }\left(m_{S}, m_{T}\right) \in R_{1},\left(m_{S}^{\prime}, m_{T}\right) \in R_{2}\right\} .
$$

By Theorem 2.8.7 of [4],

$$
\begin{aligned}
P_{S} \cap \dot{w} P_{T}^{-} \dot{w}^{-1}= & \left(M_{S} \cap \operatorname{Ad}_{\dot{w}}\left(M_{T}\right)\right)\left(M_{S} \cap \operatorname{Ad}_{\dot{w}}\left(N_{T}^{-}\right)\right)\left(N_{S} \cap \operatorname{Ad}_{\dot{w}}\left(M_{T}\right)\right) \\
& \times\left(N_{S} \cap \operatorname{Ad}_{\dot{w}}\left(N_{T}^{-}\right)\right) .
\end{aligned}
$$

Note that $M_{S} \cap \operatorname{Ad}_{\dot{w}}\left(M_{T}\right)=M_{S \cap w(T)}, M_{S} \cap \operatorname{Ad}_{\dot{w}}\left(N_{T}^{-}\right)=N_{S \cap w(T)}^{S,-}=M_{S} \cap N_{S \cap w(T)}^{-}$, and $N_{S} \cap \operatorname{Ad}_{\dot{w}}\left(M_{T}\right)=N_{T \cap w^{-1}(S)}^{T}=M_{T} \cap N_{T \cap w^{-1}(S)}$. Thus

$$
R_{1}=\left\{\left(m, \operatorname{Ad}_{\dot{w}^{-1}}(m)\right): m \in M_{S \cap w(T)}\right\}\left(N_{S \cap w(T)}^{S,-} \times N_{T \cap w^{-1}(S)}^{T}\right) .
$$

Therefore $\left(m_{S}^{\prime}, m_{S}\right) \in R_{3}$ if and only if there exist $n \in N_{S \cap w(T)}^{S,-}, n_{1} \in N_{T \cap w^{-1}(S)}^{T}$, and $m \in M_{S \cap w(T)}$ such that $m_{S}=m n$ and $\left(m_{S}^{\prime}, \operatorname{Ad}_{\dot{w}^{-1}}(m) n_{1}\right) \in R_{2}$. It follows from the definition of $R$ that $\left(m_{S}^{\prime}, m_{S}\right) \in R_{3}$ if and only if there exist $m^{\prime} \in M_{S_{w}}, m \in M_{T_{w}}, n \in N_{T_{w}}^{S,-}$, and $n^{\prime} \in N_{S_{w}}^{S}=M_{S} \cap N_{S_{w}}$ such that $m_{S}=m n, m_{S}^{\prime}=m^{\prime} n^{\prime}$ and $\left(m^{\prime}, \operatorname{Ad}_{\dot{w}}^{-1}(m)\right) \in R$. Thus 
$R_{3}=R_{\dot{w}}^{S}$. Since $C_{S} \times\{e\} \subset R_{\dot{w}}^{S}$, the (right) action of $C_{S} \times\{e\}$ on $R_{\dot{w}}^{S} \backslash\left(M_{S} \times M_{S}\right)$ is trivial. Thus we have

$$
\begin{aligned}
R_{3} \backslash & \backslash M_{S} \times M_{S} /\left(\left(M_{S}\right)_{\Delta}\left(C_{S} \times\{e\}\right)\right) \\
& \cong R_{\dot{w}}^{S} \backslash M_{S} \times M_{S} /\left(\left(M_{S}\right)_{\Delta}\left(C_{S} \times\{e\}\right)\right) \\
\cong & R_{\dot{w}}^{S} \backslash M_{S} \times M_{S} /\left(M_{S}\right)_{\Delta} \\
\cong & \left(M_{S}\right)_{\Delta} \backslash M_{S} \times M_{S} / R_{\dot{w}}^{S},
\end{aligned}
$$

where the last identification is induced by the inverse map of $M_{S} \times M_{S}$.

Combining the above identification with the identifications in (3.8)-(3.9) and the inclusion of (3.6), we get a well-defined injective map $\left(M_{S}\right)_{\Delta} \backslash M_{S} \times M_{S} / R_{\dot{w}}^{S} \rightarrow G_{\Delta} \backslash G \times G / R$ given by

$$
\left(M_{S}\right)_{\Delta}\left(m, m^{\prime}\right) R_{\dot{w}}^{S} \rightarrow\left[\left(\left(m^{\prime}\right)^{-1}, \dot{w} \theta_{d}\left(m^{-1}\right)\right)\right]=\left[\left(m^{\prime}\right)^{-1} m, \dot{w}\right]=\left[m, m^{\prime} \dot{w}\right] .
$$

This finishes the proof of Lemma 3.8.

Proof of Theorem 3.7. - By Lemma 3.8, each $\left(G_{\Delta}, R\right)$ double coset in $G \times G$ determines a unique $w \in{ }^{S} W^{T}$ and a unique double coset $\left[m, m^{\prime}\right]_{1} \in\left(M_{S}\right)_{\Delta} \backslash M_{S} \times M_{S} / R_{\dot{w}}^{S}$. Let $\left(S_{0}, T_{0}, d_{0}, w_{0}\right)=(S, T, d, w)$. By successively applying Lemma 3.8 to a sequence of smaller subgroups, we obtain a sequence $\mathbf{q}$ of quadruples $\mathbf{q}_{i}=\left(S_{i}, T_{i}, d_{i}, w_{i}\right)$ as in Notation 3.4, as well as a double coset in $\left(M_{S_{i}}\right)_{\Delta} \backslash M_{S_{i}} \times M_{S_{i}} / R_{i}$, where $R_{i}$ is the subgroup of $M_{S_{i}} \times M_{S_{i}}$ defined analogously to $R_{\dot{w}}^{S}$.

As in Notation 3.4, let $i_{0}$ be the smallest integer such that $S_{i_{0}+1}=S_{i_{0}}$ and let $v=v_{\infty}(\mathbf{q})=$ $w_{i_{0}} w_{i_{0}-1} \cdots w_{0}$. Then each $\left(G_{\Delta}, R\right)$-double coset in $G \times G$ is of the form $\left[m, m^{\prime} \dot{v}\right]$ for $m \in M_{S_{i_{0}+1}}$. By Proposition 3.5, $v \in W^{T}$, and $S_{i_{0}+1}=S(v, d)$. Moreover, $R_{i_{0}+1}=R_{\dot{v}}$ by definition. Thus double cosets in $\left(M_{S_{i_{0}+1}}\right)_{\Delta} \backslash M_{S_{i_{0}+1}} \times M_{S_{i_{0}+1}} / R_{i_{0}+1}$ coincide with double cosets in $\left(M_{S(v, d)}\right)_{\Delta} \backslash M_{S(v, d)} \times M_{S(v, d)} / R_{\dot{v}}$. It is easy to see that the map

$$
\left(M_{S(v, d)}\right)_{\Delta} \backslash M_{S(v, d)} \times M_{S(v, d)} / R_{\dot{v}} \rightarrow M_{S(v, d)} / R_{\dot{v}}:\left[m, m^{\prime}\right] \mapsto\left[m^{\prime-1} m\right]
$$

is a bijection. This proves Theorem 3.7 .

\section{3. $G_{\Delta}$-orbits in $\mathcal{L}$}

NOTATION 3.9. - For a generalized BD-triple $(S, T, d), V \in \mathcal{L}_{\text {space }}\left(\mathfrak{z}_{S} \oplus \mathfrak{z}_{T}\right), m \in M_{S(v, d)}$, $v \in W^{T}$, and $\dot{v} \in G$ a fixed representative of $v$ in $G$, set

$$
\mathfrak{l}_{S, T, d, V, \dot{v}, m}=\operatorname{Ad}_{(m, \dot{v})} \mathfrak{l}_{S, T, d, V},
$$

where $\mathfrak{l}_{S, T, d, V}$ is given in (2.4). Define

$$
R_{\dot{v}}=\left\{\left(m_{1}, m_{1}^{\prime}\right) \in M_{S(v, d)} \times M_{S(v, d)}: \gamma_{d}\left(\chi_{S}\left(m_{1}\right)\right)=\chi_{T}\left(\dot{v}^{-1} m_{1}^{\prime} \dot{v}\right)\right\},
$$

and let $R_{\dot{v}}$ act on $M_{S(v, d)}$ (from the right) by

$$
m \cdot\left(m_{1}, m_{1}^{\prime}\right)=\left(m_{1}^{\prime}\right)^{-1} m m_{1}, \quad m \in M_{S(v, d)},\left(m_{1}, m_{1}^{\prime}\right) \in R_{\dot{v}} .
$$

As an immediate corollary of Theorem 3.7, we have

$4^{\text {e }}$ SÉRIE - TOME $39-2006-\mathrm{N}^{\circ} 2$ 
COROLlARY 3.10. - Every $G_{\Delta}$-orbit in $\mathcal{L}$ passes through an $\mathfrak{l}_{S, T, d, V, \dot{v}, m}$ for a unique generalized $B D$-triple $(S, T, d)$, a unique $V \in \mathcal{L}_{\text {space }}\left(\mathfrak{z}_{S} \oplus \mathfrak{z}_{T}\right)$, a unique $v \in W^{T}$, and some $m \in M_{S(v, d)}$; Two such Lagrangian subalgebras $\mathfrak{l}_{S, T, d, V, v, m_{1}}$ and $\mathfrak{l}_{S, T, d, V, v, m_{2}}$ are in the same $G_{\Delta}$-orbit if and only if $m_{1}$ and $m_{2}$ are in the same $R_{\dot{v}}$-orbit in $M_{S(v, d)}$.

\subsection{Normalizer subalgebras of $\mathfrak{g}_{\Delta}$ at $\mathfrak{l} \in \mathcal{L}$}

For $\mathfrak{l}=\mathfrak{l}_{S, T, d, V, \dot{v}, m}$ as in Corollary 3.10, we now compute its normalizer subalgebra $\mathfrak{n}(\mathfrak{l})$ in $\mathfrak{g} \cong \mathfrak{g}_{\Delta}=\{(x, x): x \in \mathfrak{g}\}$. Introduce

$$
\phi:=\operatorname{Ad}_{\dot{v}} \gamma_{d} \chi_{S} \operatorname{Ad}_{m}^{-1}: \mathfrak{p}_{S} \rightarrow \mathfrak{g} .
$$

Consider the standard parabolic subalgebra $\mathfrak{p}_{S(v, d)}$ and its decomposition $\mathfrak{p}_{S(v, d)}=\mathfrak{z}_{S(v, d)}+$ $\mathfrak{g}_{S(v, d)}+\mathfrak{n}_{S(v, d)}$ (see Notation 2.11).

LEMMA 3.11. - The map $\phi=\operatorname{Ad}_{\dot{v}} \gamma_{d} \chi_{S} \operatorname{Ad}_{m}^{-1}$ leaves each of $\mathfrak{z}_{S(v, d)}, \mathfrak{g}_{S(v, d)}$, and $\mathfrak{n}_{S(v, d)}$ invariant. Moreover, $\phi: \mathfrak{n}_{S(v, d)} \rightarrow \mathfrak{n}_{S(v, d)}$ is nilpotent.

Proof. - Let $x \in \mathfrak{z}_{S(v, d)}$. Then $\phi(x)=\operatorname{Ad}_{\dot{v}} \gamma_{d} \chi_{S}(x) \in \mathfrak{h}$. For $\alpha \in S(v, d)$, since $(v d)^{-1} \alpha \in$ $S(v, d)$,

$$
\alpha(\phi(x))=\left((v d)^{-1} \alpha\right)\left(\chi_{S}(x)\right)=\left((v d)^{-1} \alpha\right)(x)=0 .
$$

Thus $\phi(x) \in \mathfrak{z}_{S(v, d)}$, so $\mathfrak{z}_{S(v, d)}$ is $\phi$-invariant. Since both $\operatorname{Ad}_{\dot{v}} \gamma_{d}$ and $\operatorname{Ad}_{m}^{-1}$ leave $\mathfrak{g}_{S(v, d)}$ invariant, we see that $\left.\phi\right|_{\mathfrak{g}_{S(v, d)}}=\operatorname{Ad}_{\dot{v}} \gamma_{d} \operatorname{Ad}_{m}^{-1}$ leaves $\mathfrak{g}_{S(v, d)}$ invariant.

To show that $\mathfrak{n}_{S(v, d)}$ is $\phi$-invariant and that $\phi: \mathfrak{n}_{S(v, d)} \rightarrow \mathfrak{n}_{S(v, d)}$ is nilpotent, set $\Sigma_{0}^{+}=$ $\Sigma^{+}-[S]$, and for $j \geqslant 1$, set

$$
\Sigma_{j}^{+}=\left\{\alpha \in \Sigma^{+}: \alpha \in[S], v d \alpha \in[S], \ldots,(v d)^{j-1}(\alpha) \in[S],(v d)^{j}(\alpha) \notin[S]\right\} .
$$

Then $\Sigma^{+}-[S(v, d)]=\bigcup_{j \geqslant 0} \Sigma_{j}^{+}$and $v d\left(\Sigma_{j}^{+}\right) \subset \Sigma_{j-1}^{+}$for $j \geqslant 1$. For $j \geqslant 0$, set $\mathfrak{n}_{j}=\bigoplus_{\alpha \in \Sigma_{j}^{+}} \mathfrak{g}_{\alpha}$. Then $\mathfrak{n}_{0}=\mathfrak{n}_{S}$, and $\mathfrak{n}_{S(v, d)}=\sum_{j \geqslant 0} \mathfrak{n}_{j}$ is a finite direct sum. It is easy to prove by induction on $j$ that

$$
\alpha \in \Sigma_{j}^{+}, \beta \in[S(v, d)], \alpha+\beta \in \Sigma \Longrightarrow \alpha+\beta \in \Sigma_{j}^{+}, \quad \forall j \geqslant 0 .
$$

It follows that $\left[\mathfrak{m}_{S(v, d)}, \mathfrak{n}_{j}\right] \subset \mathfrak{n}_{j}$ for each $j \geqslant 0$. Thus $\operatorname{Ad}_{m} \mathfrak{n}_{j}=\mathfrak{n}_{j}, \forall j \geqslant 0$. By setting $\mathfrak{n}_{-1}=0$, we then have $\operatorname{Ad}_{i} \gamma_{d} \chi_{S}\left(\mathfrak{n}_{j}\right) \subset \mathfrak{n}_{j-1}, \forall j \geqslant 0$. Thus $\phi\left(\mathfrak{n}_{j}\right) \subset \mathfrak{n}_{j-1}$ for all $j \geqslant 0$, and $\phi: \mathfrak{n}_{S(v, d)} \rightarrow \mathfrak{n}_{S(v, d)}$ is nilpotent.

Remark 3.12. - Similar arguments imply the same statements for $\mathfrak{n}_{S(v, d)}^{-}$. In particular, for $j \geqslant 0$, set $\mathfrak{n}_{j}^{-}=\bigoplus_{\alpha \in \Sigma_{j}^{+}} \mathfrak{g}_{-\alpha}$ and set $\mathfrak{n}_{-1}^{-}=0$. Then $\phi\left(\mathfrak{n}_{j}^{-}\right) \subset \mathfrak{n}_{j-1}^{-}$for $j \geqslant 0, \mathfrak{n}_{S}^{-}=\mathfrak{n}_{0}^{-}$, and $\mathfrak{n}_{S(v, d)}^{-}=\sum_{j \geqslant 0} \mathfrak{n}_{j}^{-}$is a direct sum.

Since $\phi: \mathfrak{n}_{S(v, d)} \rightarrow \mathfrak{n}_{S(v, d)}$ is nilpotent, we can define

$$
\psi:=(1-\phi)^{-1}=1+\phi+\phi^{2}+\phi^{3}+\cdots: \mathfrak{n}_{S(v, d)} \rightarrow \mathfrak{n}_{S(v, d)} .
$$

Let $\Sigma_{v}^{+}=\left\{\alpha \in \Sigma^{+}: v^{-1} \alpha \in \Sigma^{-}\right\}$. Since $v\left([T] \cap \Sigma^{+}\right) \subset \Sigma^{+}$, we have $\Sigma_{v}^{+} \subset \Sigma^{+}-[S(v, d)]$. Let

$$
\mathfrak{n}_{v}=\bigoplus_{\alpha \in \Sigma_{v}^{+}} \mathfrak{g}_{\alpha}=\mathfrak{n} \cap \operatorname{Ad}_{\dot{v}}\left(\mathfrak{n}^{-}\right)
$$

ANNALES SCIENTIFIQUES DE L'ÉCOLE NORMALE SUPÉRIEURE 
Then $\mathfrak{n}_{v} \subset \mathfrak{n}_{S(v, d)}$.

THEOREM 3.13. - The normalizer subalgebra $\mathfrak{n}(\mathfrak{l})$ in $\mathfrak{g}_{\Delta} \cong \mathfrak{g}$ of $\mathfrak{l}=\mathfrak{l}_{S, T, d, V, \dot{v}, m}$ in (3.11) is

$$
\mathfrak{n}(\mathfrak{l})=\mathfrak{z}_{S(v, d)}^{\prime}+\mathfrak{g}_{S(v, d)}^{\phi}+\psi\left(\mathfrak{n}_{v}\right)
$$

where $\mathfrak{g}_{S(v, d)}^{\phi}$ is the fixed point set of $\left.\phi\right|_{\mathfrak{g}_{S(v, d)}}=\operatorname{Ad}_{\dot{v}} \gamma_{d} \operatorname{Ad}_{m}^{-1}$ in $\mathfrak{g}_{S(v, d)}$, and

$$
\begin{aligned}
\mathfrak{z}_{S(v, d)}^{\prime} & =\left\{z \in \mathfrak{z}_{S(v, d)}: z-\phi(z) \in \operatorname{Ad}_{\dot{v}} \mathfrak{z}_{T}\right\} \\
& =\left\{z \in \mathfrak{z}_{S(v, d)}: \gamma_{d} \chi_{S}(z)=\chi_{T}\left(\operatorname{Ad}_{\dot{v}}^{-1} z\right)\right\} .
\end{aligned}
$$

Proof. - The normalizer subgroup $R_{S, T, d}$ of $\mathfrak{l}_{S, T, d, V}$ in $G \times G$ has Lie algebra

$$
\begin{aligned}
\mathfrak{r}_{S, T, d} & =\left(\mathfrak{z}_{S} \oplus \mathfrak{z}_{T}\right)+\left(\mathfrak{n}_{S} \oplus \mathfrak{n}_{T}^{-}\right)+\left\{\left(x, \gamma_{d}(x)\right): x \in \mathfrak{g}_{S}\right\} \\
& =\left\{(x, y) \in \mathfrak{p}_{S} \oplus \mathfrak{p}_{T}^{-}: \gamma_{d} \chi_{S}(x)=\chi_{T}(y)\right\} .
\end{aligned}
$$

Since $\mathfrak{l}=\operatorname{Ad}_{(m, \dot{v})} \mathfrak{l}_{S, T, d, V}$, it follows that $\mathfrak{n}(\mathfrak{l})=\left\{x \in \mathfrak{g}:\left(\operatorname{Ad}_{m}^{-1} x, \operatorname{Ad}_{\dot{v}}^{-1} x\right) \in \mathfrak{r}_{S, T, d}\right\}$. Thus $x \in \mathfrak{n}(\mathfrak{l})$ if and only if $x \in \mathfrak{p}_{S} \cap \operatorname{Ad}_{\dot{v}} \mathfrak{p}_{T}^{-}$and $\gamma_{d} \chi_{S}\left(\operatorname{Ad}_{m}^{-1}(x)\right)=\chi_{T}\left(\operatorname{Ad}_{\dot{v}}^{-1} x\right)$, which is equivalent to

$$
x-\operatorname{Ad}_{\dot{v}} \gamma_{d} \chi_{S}\left(\operatorname{Ad}_{m}^{-1}(x)\right) \in \operatorname{Ad}_{\dot{v}}\left(\mathfrak{z}_{T}+\mathfrak{n}_{T}^{-}\right) .
$$

Let $\chi_{S}$ also denote the projection $\mathfrak{g} \rightarrow \mathfrak{g}_{S}$ with respect to the decomposition $\mathfrak{g}=\mathfrak{n}_{S}^{-}+\mathfrak{z} S+\mathfrak{g}_{S}+$ $\mathfrak{n}_{S}$, so $\phi: x \mapsto \operatorname{Ad}_{\dot{v}} \gamma_{d} \chi_{S}\left(\operatorname{Ad}_{m}^{-1} x\right)$ is defined for all $x \in \mathfrak{g}$. Let $\mathfrak{c}$ be the set of all $x \in \mathfrak{g}$ satisfying (3.16). Then $\mathfrak{n}(\mathfrak{l})=\mathfrak{c} \cap\left(\mathfrak{p}_{S} \cap \operatorname{Ad}_{\dot{v}} \mathfrak{p}_{T}^{-}\right)$.

Consider the decomposition $\mathfrak{g}=\mathfrak{n}_{S(v, d)}^{-}+\mathfrak{m}_{S(v, d)}+\mathfrak{n}_{S(v, d)}$. Since $\alpha \notin v([T])$ implies that $\alpha \notin[S(v, d)]$, we have

$$
\operatorname{Ad}_{\dot{v}} \mathfrak{n}_{T}^{-}=\left(\operatorname{Ad}_{\dot{v}} \mathfrak{n}_{T}^{-}\right) \cap \mathfrak{n}^{-}+\left(\operatorname{Ad}_{\dot{v}} \mathfrak{n}_{T}^{-}\right) \cap \mathfrak{n} \subset \mathfrak{n}_{S(v, d)}^{-}+\mathfrak{n}_{S(v, d)}
$$

Moreover, it is easy to see that $\left(\operatorname{Ad}_{\dot{v}} \mathfrak{n}_{T}^{-}\right) \cap \mathfrak{n}=\left(\operatorname{Ad}_{\dot{v}} \mathfrak{n}_{T}^{-}\right) \cap \mathfrak{n}_{S(v, d)}=\mathfrak{n}_{v}$, so

$$
\operatorname{Ad}_{\dot{v}} \mathfrak{n}_{T}^{-}=\left(\operatorname{Ad}_{\dot{v}} \mathfrak{n}_{T}^{-}\right) \cap \mathfrak{n}_{S(v, d)}^{-}+\mathfrak{n}_{v}
$$

Now let $x \in \mathfrak{g}$ and write $x=x_{-}+x_{0}+x_{+}$, where $x_{-} \in \mathfrak{n}_{S(v, d)}^{-}, x_{0} \in \mathfrak{m}_{S(v, d)}$, and $x_{+} \in \mathfrak{n}_{S(v, d)}$. It follows from Lemma 3.11 and (3.17) that $x \in \mathfrak{c}$, i.e., $x$ satisfies (3.16), if and only if

$$
\left\{\begin{array}{l}
x_{0}-\phi\left(x_{0}\right) \in \operatorname{Ad}_{\dot{v}} \mathfrak{z}_{T}, \\
x_{+}-\phi\left(x_{+}\right) \in \mathfrak{n}_{v} \\
x_{-}-\phi\left(x_{-}\right) \in\left(\operatorname{Ad}_{\dot{v}} \mathfrak{n}_{T}^{-}\right) \cap \mathfrak{n}_{S(v, d)}^{-}
\end{array}\right.
$$

Write $x_{0}=z_{0}+y_{0}$, where $z_{0} \in \mathfrak{z}_{S(v, d)}$ and $y_{0} \in \mathfrak{g}_{S(v, d)}$. Since $\operatorname{Ad}_{\dot{v}} \mathfrak{z}_{T} \subset \mathfrak{z}_{S(v, d)}$ and since both $\mathfrak{z}_{S(v, d)}$ and $\mathfrak{g}_{S(v, d)}$ are $\phi$-invariant, $x_{0}-\phi\left(x_{0}\right) \in \operatorname{Ad}_{\dot{v}} \mathfrak{z}_{T}$ if and only if $z_{0}-\phi\left(z_{0}\right) \in \operatorname{Ad}_{\dot{v}} \mathfrak{z}_{T}$ and $y_{0}-\phi\left(y_{0}\right)=0$, which is the same as $x_{0} \in \mathfrak{z}_{S(v, d)}^{\prime}+\mathfrak{g}_{S(v, d)}^{\phi}$. Recall that $\psi=(1-\phi)^{-1}$ on $\mathfrak{n}_{S(v, d)}$. Thus, $x_{+}-\phi\left(x_{+}\right) \in \mathfrak{n}_{v}$ if and only if $x_{+} \in \psi\left(\mathfrak{n}_{v}\right)$. Since $\mathfrak{n}_{v} \subset \mathfrak{n}_{S(v, d)}$,

$$
\psi\left(\mathfrak{n}_{v}\right) \subset \mathfrak{p}_{S} \cap\left(\mathfrak{n}_{v}+\phi\left(\mathfrak{n}_{S(v, d)}\right)\right) \subset \mathfrak{p}_{S} \cap\left(\operatorname{Ad}_{\dot{v}}\left(\mathfrak{n}^{-}+\mathfrak{m}_{T}\right)\right) \subset \mathfrak{p}_{S} \cap \operatorname{Ad}_{\dot{v}} \mathfrak{p}_{T}^{-}
$$

$4^{\mathrm{e}}$ SÉRIE - TOME $39-2006-\mathrm{N}^{\circ} 2$ 
Note that $\mathfrak{z}_{S(v, d)}^{\prime}+\mathfrak{g}_{S(v, d)}^{\phi} \subset \mathfrak{p}_{S} \cap \operatorname{Ad}_{\dot{v}} \mathfrak{p}_{T}^{-}$. Thus $\mathfrak{c} \cap\left(\mathfrak{p}_{S} \cap \operatorname{Ad}_{\dot{v}} \mathfrak{p}_{T}^{-}\right)=\mathfrak{z}_{S(v, d)}^{\prime}+\mathfrak{g}_{S(v, d)}^{\phi}+$ $\psi\left(\mathfrak{n}_{v}\right)+\mathfrak{c}^{\prime}$, where $\mathfrak{c}^{\prime}$ consists of all

$$
x_{-} \in \mathfrak{n}_{S(v, d)}^{-} \cap \mathfrak{p}_{S} \cap \operatorname{Ad}_{\dot{v}} \mathfrak{p}_{T}^{-} \subset \mathfrak{n}_{S(v, d)}^{-} \cap \mathfrak{m}_{S}
$$

satisfying the third condition in (3.18). It suffices to show that $\mathfrak{c}^{\prime}=0$.

We regard the direct sum decomposition $\mathfrak{n}_{S(v, d)}^{-}=\sum_{j \geqslant 0} \mathfrak{n}_{j}^{-}$from Remark 3.12 as a grading of $\mathfrak{n}_{S(v, d)}^{-}$. Let $U=\mathfrak{n}_{S(v, d)}^{-} \cap \mathfrak{m}_{S}=\sum_{j>0} \mathfrak{n}_{j}^{-}$and let $Y=\operatorname{Ad}_{i} \mathfrak{n}_{T}^{-} \cap \mathfrak{n}_{S(v, d)}^{-}$. Clearly, $U$ and $Y$ are graded subspaces of $\mathfrak{n}_{S(v, d)}^{-}$, since they are sums of root spaces. Since $U \subset \mathfrak{g}_{S}, \phi$ is injective on $U$. Moreover, the image of $\phi$ is in $\operatorname{Ad}_{\dot{v}}\left(\mathfrak{g}_{T}\right)$ so $Y$ has zero intersection with the image of $\phi$. The fact that $\mathfrak{c}^{\prime}=0$ now follows from the following simple linear algebra fact in Lemma 3.14.

LEMMA 3.14. - Let $V=\bigoplus V_{i}$ be a graded vector space with graded subspaces $U$ and $Y$. Let $\phi$ be an endomorphism of $V$ such that

(1) $\phi\left(V_{i}\right) \subset V_{i-1}$ for all $i$,

(2) $Y \cap \operatorname{Im}(\phi)=0$, and

(3) $U \cap \operatorname{Ker}(\phi)=0$.

Then $\{v \in U: v-\phi(v) \in Y\}=0$.

Remark 3.15. - Theorem 3.13 implies that

$$
\mathfrak{n}(\mathfrak{l}) \subset \mathfrak{p}_{S(v, d)} \cap \operatorname{Ad}_{\dot{v}} \mathfrak{p}_{T}^{-}=\mathfrak{p}_{S(v, d)} \cap \operatorname{Ad}_{\dot{v}} \mathfrak{p}_{T}^{-}=\mathfrak{m}_{S(v, d)}+\mathfrak{n}_{v}+\mathfrak{n}_{S(v, d)} \cap \operatorname{Ad}_{\dot{v}} \mathfrak{m}_{T}
$$

and that $\mathfrak{n}(\mathfrak{l})=\mathfrak{n}(\mathfrak{l}) \cap \mathfrak{m}_{S(v, d)}+\mathfrak{n}(\mathfrak{l}) \cap\left(\mathfrak{n}_{v}+\mathfrak{n}_{S(v, d)} \cap \operatorname{Ad}_{\dot{v}} \mathfrak{m}_{T}\right)$. Further,

$$
\left(\mathfrak{n}(\mathfrak{l}) \cap \mathfrak{m}_{S(v, d)}\right)_{\Delta}=\left(\mathfrak{z}_{S(v, d)}^{\prime}+\mathfrak{g}_{S(v, d)}^{\phi}\right)_{\Delta}=\left(\mathfrak{m}_{S(v, d)}\right)_{\Delta} \cap \operatorname{Ad}_{(m, \dot{v})} \mathfrak{r}_{S, T, d}
$$

and $\mathfrak{n}(\mathfrak{l}) \cap\left(\mathfrak{n}_{v}+\mathfrak{n}_{S(v, d)} \cap \operatorname{Ad}_{\dot{v}} \mathfrak{m}_{T}\right)$ is the graph of the map

$$
\psi-1=\phi \psi=\phi+\phi^{2}+\cdots: \mathfrak{n}_{v} \rightarrow \mathfrak{n}_{S(v, d)} \cap \operatorname{Ad}_{\dot{v}} \mathfrak{m}_{T} .
$$

In [24], the map $\psi-1$ is shown to be related to some set-theoretical solutions to the Quantum Yang-Baxter Equation.

\subsection{Intersections of $\mathfrak{g}_{\Delta}$ with $\mathfrak{l} \in \mathcal{L}$}

By Corollary 3.10, to compute $\mathfrak{g}_{\Delta} \cap \mathfrak{l}$ for any $\mathfrak{l} \in \mathcal{L}$, we may assume that $\mathfrak{l}=\mathfrak{l}_{S, T, d, V, \dot{v}, m}$ as given in (3.11).

Proposition 3.16. - For the Lagrangian subalgebra $\mathfrak{l}_{S, T, d, V, \dot{v}, m}$ as given in (3.11), let the notation be as in Theorem 3.13. Then

$$
\mathfrak{g}_{\Delta} \cap \mathfrak{l}_{S, T, d, V, \dot{v}, m}=\operatorname{Ad}_{(m, \dot{v})} V^{\prime}+\left(\mathfrak{g}_{S(v, d)}^{\phi}+\psi\left(\mathfrak{n}_{v}\right)\right)_{\Delta},
$$

where $V^{\prime}=\left\{\left(z, v^{-1} z\right): z \in \mathfrak{z}_{S(v, d)}^{\prime}\right\} \cap\left(V+\left\{\left(x, \gamma_{d}(x)\right): x \in \mathfrak{h}_{S}\right\}\right)$.

Proof. - Set $\mathfrak{l}=\mathfrak{l}_{S, T, d, V, \dot{v}, m}$. By Theorem 3.13,

$$
\mathfrak{g}_{\Delta} \cap \mathfrak{l} \subset \mathfrak{n}(\mathfrak{l})=\left(\mathfrak{z}_{S(v, d)}^{\prime}+\mathfrak{g}_{S(v, d)}^{\phi}+\psi\left(\mathfrak{n}_{v}\right)\right)_{\Delta} .
$$


Since $\left(\mathfrak{g}_{S(v, d)}^{\phi}+\psi\left(\mathfrak{n}_{v}\right)\right)_{\Delta} \subset \mathfrak{l}$, we see that

$$
\mathfrak{g}_{\Delta} \cap \mathfrak{l}=\left(\left(\mathfrak{z}_{S(v, d)}^{\prime}\right)_{\Delta} \cap \mathfrak{l}\right)+\left(\mathfrak{g}_{S(v, d)}^{\phi}+\psi\left(\mathfrak{n}_{v}\right)\right)_{\Delta},
$$

and

$$
\left(\mathfrak{z}_{S(v, d)}^{\prime}\right)_{\Delta} \cap \mathfrak{l}=\left(\mathfrak{z}_{S(v, d)}^{\prime}\right)_{\Delta} \cap \mathfrak{l} \cap(\mathfrak{h} \oplus \mathfrak{h})=\operatorname{Ad}_{(m, \dot{v})} V^{\prime} .
$$

Recall that a Belavin-Drinfeld triple [3] for $\mathfrak{g}$ is a generalized Belavin-Drinfeld triple $(S, T, d)$ with the nilpotency condition: for every $\alpha \in S$, there exists an integer $n \geqslant 1$ such that $\alpha, d \alpha, \ldots, d^{n-1} \alpha \in S$ but $d^{n} \alpha \notin S$. The nilpotency condition is equivalent to $S(1, d)=\emptyset$, where 1 is the identity element in the Weyl group $W$.

Definition 3.17. - A Belavin-Drinfeld system is a quadruple $(S, T, d, V)$, where $(S, T, d)$ is a Belavin-Drinfeld triple, and $V$ is a Lagrangian subspace of $\mathfrak{z}_{S} \oplus \mathfrak{z}_{T}$ such that

$$
\mathfrak{h}_{\Delta} \cap\left(V+\left\{\left(x, \gamma_{d}(x)\right): x \in \mathfrak{h}_{S}\right\}\right)=0 .
$$

We now derive a theorem of Belavin and Drinfeld [3] from Proposition 3.16.

COROLlARY 3.18 (Belavin-Drinfeld). - A Lagrangian subalgebra $\mathfrak{l}$ of $\mathfrak{g} \oplus \mathfrak{g}$ has trivial intersection with $\mathfrak{g}_{\Delta}$ if and only if $\mathfrak{l}$ is $G_{\Delta}$-conjugate to a Lagrangian subalgebra of the form $\mathfrak{l}_{S, T, d, V}$, where $(S, T, d, V)$ is a Belavin-Drinfeld system.

Proof. - It is clear from Proposition 3.16 that $\mathfrak{g}_{\Delta} \cap \mathfrak{l}_{S, T, d, V}=0$ if $(S, T, d, V)$ is a BelavinDrinfeld system. Suppose that $\mathfrak{g}_{\Delta} \cap \mathfrak{l}_{S, T, d, V, \dot{v}, m}=0$, where $\mathfrak{l}_{S, T, d, V, \dot{v}, m}$ is as in (3.11). Since $\operatorname{dim} \psi\left(\mathfrak{n}_{v}\right)=l(v)$, the length of $v$, and since every automorphism of a semi-simple Lie algebra has fixed point set of dimension at least one [32], $v=1$ and $S(1, d)=\emptyset$. In this case, $V^{\prime}$ as in Proposition 3.16 is given by

$$
V^{\prime}=\mathfrak{h}_{\Delta} \cap\left(V+\left\{\left(x, \gamma_{d}(x)\right): x \in \mathfrak{h}_{S}\right\}\right),
$$

so $\mathfrak{h}_{\Delta} \cap\left(V+\left\{\left(x, \gamma_{d}(x)\right): x \in \mathfrak{h}_{S}\right\}\right)=0$, and we have $\mathfrak{l}_{S, T, d, V, \dot{v}, m}=\operatorname{Ad}_{(m, \dot{v})} \mathfrak{l}_{S, T, d, V}$ for some $m \in H$ and $\dot{v} \in H$. Note that in this case

$$
R_{\dot{v}}=\left\{\left(h_{1}, h_{2}\right) \in H \times H: \gamma_{d}\left(\chi_{S}\left(h_{1}\right)\right)=\chi_{T}\left(h_{2}\right)\right\}
$$

and $R_{\dot{v}}$ acts on $H$ from the right by $h \cdot\left(h_{1}, h_{2}\right)=h h_{1} h_{2}^{-1}$, where $h \in H$ and $\left(h_{1}, h_{2}\right) \in R_{\dot{v}}$. Consider $\mathbf{m}: R_{\dot{v}} \rightarrow H:\left(h_{1}, h_{2}\right) \mapsto h_{1} h_{2}^{-1}$. The assumption $V^{\prime}=0$ implies that the dimension of the kernel of the differential of $\mathbf{m}$ is less than or equal to $\operatorname{dim}\left(\mathfrak{z}_{T}\right)$. It follows that the differential of $\mathbf{m}$ is onto, so $\mathbf{m}$ is onto. By Corollary $3.10, \mathfrak{l}_{S, T, d, V, \dot{v}, m}$ is in the $G_{\Delta}$-orbit of $\mathfrak{l}_{S, T, d, V}$.

\subsection{Examples of smooth $G_{\Delta}$-orbit closures in $\mathcal{L}$}

The closure of a $G_{\Delta}$-orbit in $\mathcal{L}$ is not necessarily smooth. We now look at two cases for which such a closure is smooth.

PROPOSITION 3.19. - If $\mathfrak{l} \in \mathcal{L}$ is such that $\mathfrak{g}_{\Delta} \cap \mathfrak{l}=0$, then $\overline{G_{\Delta} \cdot \mathfrak{l}}=\overline{(G \times G) \cdot \mathfrak{l}}$ is smooth.

Proof. - We only need to show that $\operatorname{dim}\left(G_{\Delta} \cdot \mathfrak{l}\right)=\operatorname{dim}((G \times G) \cdot \mathfrak{l})$. By Corollary 3.18, we may assume that $\mathfrak{l}=\mathfrak{l}_{S, T, d, V}$, where $(S, T, d, V)$ is a Belavin-Drinfeld system, and so

$$
\mathfrak{g}_{\Delta} \cap \mathfrak{r}_{S, T, d}=\mathfrak{h}_{\Delta} \cap\left(\left(\mathfrak{z}_{S} \oplus \mathfrak{z}_{T}\right)+V_{S}\right),
$$

$4^{\text {e }}$ SÉRIE - TOME $39-2006-\mathrm{N}^{\circ} 2$ 
where $V_{S}=\left\{\left(x, \gamma_{d}(x)\right): x \in \mathfrak{h}_{S}\right\}$. For a subspace $A$ of $\mathfrak{h} \oplus \mathfrak{h}$, let

$$
A^{\perp}=\left\{(x, y) \in \mathfrak{h} \oplus \mathfrak{h}:\left\langle(x, y),\left(x_{1}, y_{1}\right)\right\rangle=0 \forall\left(x_{1}, y_{1}\right) \in A\right\} .
$$

Then $\left.\left(\mathfrak{h}_{\Delta} \cap\left(\left(\mathfrak{z}_{S} \oplus \mathfrak{z}_{T}\right)+V_{S}\right)\right)\right)^{\perp}=\mathfrak{h}_{\Delta}+V_{S}$. Since $\mathfrak{h}_{\Delta} \cap V_{S}=0$, we have

$$
\left.\operatorname{dim}\left(\mathfrak{h}_{\Delta} \cap\left(\left(\mathfrak{z}_{S} \oplus \mathfrak{z}_{T}\right)+V_{S}\right)\right)\right)=2 \operatorname{dim} \mathfrak{h}-\operatorname{dim} \mathfrak{h}-\operatorname{dim} \mathfrak{h}_{S}=\operatorname{dim} \mathfrak{z} S .
$$

Thus $\operatorname{dim}\left(G_{\Delta} \cdot \mathfrak{l}\right)=\operatorname{dim} \mathfrak{g}-\operatorname{dim} \mathfrak{z}_{S}=\operatorname{dim}((G \times G) \cdot \mathfrak{l})$ by Proposition 2.17.

We now show that the De Concini-Procesi compactification of a complex symmetric space of $G$ can be embedded into $\mathcal{L}$ as the closure of a $G_{\Delta}$-orbit in $\mathcal{L}$.

Let $\sigma: \mathfrak{g} \rightarrow \mathfrak{g}$ be an involution with lift $\sigma$ to $G$, and let $\mathfrak{g}^{\sigma}$ and $G^{\sigma}$ be the fixed subalgebra and subgroup of $\sigma$. Let again $\mathfrak{l}_{\sigma} \in \mathcal{L}$ be the graph of $\sigma$. The orbit $G_{\Delta} \cdot \mathfrak{l}_{\sigma}$ may be identified with the complex symmetric space $G / G^{\sigma}$. We will show that the closure $\overline{G_{\Delta} \cdot \mathfrak{l}_{\sigma}}$ is isomorphic to the De Concini-Procesi compactification of $G / G^{\sigma}$, which as defined as follows. Let $\operatorname{dim}\left(\mathfrak{g}^{\sigma}\right)=m$, so $\mathfrak{g}^{\sigma} \in \operatorname{Gr}(m, \mathfrak{g})$. Then $G \cdot \mathfrak{g}^{\sigma} \cong G / G^{\sigma}$, and $X_{\sigma}:=\overline{G \cdot \mathfrak{g}^{\sigma}}$, the closure of $G \cdot \mathfrak{g}^{\sigma}$ in $\operatorname{Gr}(m, \mathfrak{g})$, is the De Concini-Procesi compactification. It is smooth with finitely many $G$-orbits [5].

We recall some basic results about involutions. Choose a $\sigma$-stable maximal split Cartan subalgebra $\mathfrak{h}_{s}$ of $\mathfrak{g}$, i.e., a $\sigma$-stable Cartan subalgebra $\mathfrak{h}_{s}$ such that $\mathfrak{h}_{s}^{-\sigma}$ has maximal dimension. There is an induced action of $\sigma$ on the roots of $\mathfrak{h}_{s}$ in $\mathfrak{g}$, and there is a positive root system $\Sigma^{+}\left(\mathfrak{h}_{s}\right)$ for $\mathfrak{h}_{s}$ with the property that if $\alpha \in \Sigma^{+}\left(\mathfrak{h}_{s}\right)$, then either $\sigma(\alpha)=\alpha$ and $\left.\sigma\right|_{\mathfrak{g}_{\alpha}}=\mathrm{id}$, or $\sigma(\alpha) \notin \Sigma^{+}\left(\mathfrak{h}_{s}\right)$. A weight $\lambda \in \mathfrak{h}_{s}^{*}$ is called a regular special dominant weight if $\lambda$ is nonnegative on roots in $\Sigma^{+}\left(\mathfrak{h}_{s}\right), \sigma(\lambda)=-\lambda$, and $\lambda\left(H_{\alpha}\right)=0$ for $\alpha$ simple implies that $\sigma(\alpha)=\alpha$. If $\lambda$ and $\mu$ are weights, we say $\lambda \geqslant \mu$ if $\lambda-\mu=\sum_{\alpha \in \Sigma^{+}\left(\mathfrak{h}_{s}\right), n_{\alpha} \geqslant 0} n_{\alpha} \alpha$. For a weight $\mu$, let $\bar{\mu}=\frac{1}{2}(\mu-\sigma(\mu))$.

Lemma 3.20. - (De Concini-Procesi [5, Lemmas 4.1 and 6.1].) Let $V$ be a representation of $G$, and suppose there exists a vector $v \in V$ such that $G^{\sigma}$ is the stabilizer of the line through $v$. Suppose that when we decompose $v$ into a sum of weight vectors for $\mathfrak{h}_{s}, v=v_{\lambda}+\sum v_{i}$ where $v_{\lambda}$ has regular special dominant weight $\lambda$ and each $v_{i}$ has weight $\mu_{i}$ where $\lambda \geqslant \overline{\mu_{i}}$. Let $[v]$ be class of $v$ in $\operatorname{Proj}(V)$ and let $X^{\prime}$ be the closure of $G \cdot[v]$ in $\operatorname{Proj}(V)$. Then $X^{\prime} \cong X_{\sigma}$.

PROPOSITION 3.21. - There is a G-equivariant isomorphism $\overline{G_{\Delta} \cdot \mathfrak{l}_{\sigma}} \cong X_{\sigma}$.

Proof. - To apply Lemma 3.20, let $n=\operatorname{dim}(\mathfrak{g})$ and consider the diagonal action of $G$ on $V=\Lambda^{n}(\mathfrak{g} \oplus \mathfrak{g})$ and the vector $v_{\sigma}=\bigwedge^{n}\left(\mathfrak{l}_{\sigma}\right)$. In order to represent $v_{\sigma}$ as a sum of weight vectors in $\Lambda^{n}(\mathfrak{g} \oplus \mathfrak{g})$, we choose a basis. Let $U_{1}, \ldots, U_{l}$ be a basis of $\mathfrak{h}_{s}$. Let $\beta_{1}, \ldots, \beta_{s}$ be the roots of $\Sigma^{+}\left(\mathfrak{h}_{s}\right)$ such that $\sigma\left(\beta_{i}\right)=\beta_{i}$, and let $\alpha_{1}, \ldots, \alpha_{t}$ be the other roots in $\Sigma^{+}\left(\mathfrak{h}_{s}\right)$. For each root $\alpha$, choose a root vector $X_{\alpha}$. Then

$$
\begin{aligned}
\left\{\left(U_{i}, \sigma\left(U_{i}\right)\right) \mid i=1, \ldots, l\right\} & \cup\left\{\left(X_{ \pm \beta_{i}}, X_{ \pm \beta_{i}}\right) \mid i=1, \ldots, s\right\} \\
& \cup\left\{\left(X_{ \pm \alpha_{j}}, \sigma\left(X_{ \pm \alpha_{j}}\right)\right) \mid i=1, \ldots, t\right\}
\end{aligned}
$$

is clearly a basis of $\mathfrak{l}_{\sigma}$. Now $v_{\sigma}$ is the wedge of the vectors $\left(Y_{i}, \sigma\left(Y_{i}\right)\right)$ as $Y_{i}$ runs through the above basis, and $v_{\sigma}$ contains the summand

$$
u: \bigwedge_{i=1, \ldots, l}\left(U_{i}, \sigma\left(U_{i}\right)\right) \bigwedge_{i=1, \ldots, s}\left(X_{\beta_{i}}, 0\right) \wedge\left(X_{-\beta_{i}}, 0\right) \bigwedge_{j=1, \ldots, t}\left(X_{\alpha_{i}}, 0\right) \wedge\left(0, \sigma\left(X_{-\alpha_{i}}\right)\right)
$$

It is easy to see that $u$ is a weight vector for the diagonal Cartan subalgebra with weight $\nu:=\sum_{i=1, \ldots, t} \alpha_{i}-\sigma\left(\alpha_{i}\right)$, and $\nu=2 \sum_{i=1, \ldots, t} \alpha_{i}$ on the subspace $\mathfrak{h}_{s}^{-\sigma}$. Thus, $\nu$ is a regular special dominant weight by Lemma 6.1 in [5]. Moreover, the other weight vectors appearing in 
$v_{\sigma}$ have weights $\psi$ such that $\bar{\psi}$ is of the form $\nu-\sum_{n_{\alpha} \geqslant 0, \alpha \in \Sigma^{+}\left(\mathfrak{h}_{s}\right)} n_{\alpha} \alpha$. Thus, by Lemma 3.20, $\overline{G \cdot v_{\sigma}} \cong X_{\sigma}$.

Note that using the Plucker embedding of $\operatorname{Gr}(n, \mathfrak{g} \oplus \mathfrak{g}) \hookrightarrow \operatorname{Proj}(V)$, we can identify $\overline{G \cdot v_{\sigma}}$ with $\overline{G_{\Delta} \cdot \mathfrak{l}_{\sigma}}$. Thus, $\overline{G_{\Delta} \cdot \mathfrak{l}_{\sigma}} \cong X_{\sigma}$.

Remark 3.22. - Let $d$ be the automorphism of the Dynkin diagram of $\mathfrak{g}$ such that $\sigma=\gamma_{d} \operatorname{Ad}_{g_{0}}$ for some $g_{0}$. Consider the embedding

$$
G / G^{\sigma} \rightarrow G: g G^{\sigma} \mapsto \gamma_{d}^{-1}(g) g_{0} g^{-1}
$$

which in turn gives an embedding of $G / G^{\sigma}$ into the De Concini-Procesi compactification $Z_{d}$ of $G$. Proposition 3.21 then says that the closure of $G / G^{\sigma}$ in $Z_{d}$ is isomorphic to the De ConciniProcesi compactification of $G / G^{\sigma}$.

\section{The Poisson structure $\Pi_{0}$ on $\mathcal{L}$}

\subsection{Lagrangian splittings of $\mathfrak{g} \oplus \mathfrak{g}$}

By a Lagrangian splitting of $\mathfrak{g} \oplus \mathfrak{g}$ we mean a decomposition $\mathfrak{g} \oplus \mathfrak{g}=\mathfrak{l}_{1}+\mathfrak{l}_{2}$, where $\mathfrak{l}_{1}$ and $\mathfrak{l}_{2}$ are Lagrangian subalgebras of $\mathfrak{g} \oplus \mathfrak{g}$. By [9], every Lagrangian splitting $\mathfrak{g} \oplus \mathfrak{g}=\mathfrak{l}_{1}+\mathfrak{l}_{2}$ gives rise to a Poisson structure $\Pi_{\mathfrak{l}_{1}, \mathfrak{l}_{2}}$ on $\mathcal{L}$ as follows: let $\left\{x_{j}\right\}$ be a basis for $\mathfrak{l}_{1}$ and $\left\{\xi_{j}\right\}$ the basis for $\mathfrak{l}_{2}$ such that $\left\langle x_{j}, \xi_{k}\right\rangle=\delta_{j k}$ for $1 \leqslant j, k \leqslant n=\operatorname{dim} \mathfrak{g}$. Set

$$
R=\frac{1}{2} \sum_{j=1}^{n}\left(\xi_{j} \wedge x_{j}\right) \in \bigwedge^{2}(\mathfrak{g} \oplus \mathfrak{g}) .
$$

The action of $G \times G$ on $\mathcal{L}$ defines a Lie algebra anti-homomorphism $\kappa$ from $\mathfrak{g} \oplus \mathfrak{g}$ to the space of vector fields on $\mathcal{L}$. Set

$$
\Pi_{\mathfrak{l}_{1}, \mathfrak{l}_{2}}=(\kappa \wedge \kappa)(R)=\frac{1}{2} \sum_{j=1}^{n}\left(\kappa\left(\xi_{j}\right) \wedge \kappa\left(x_{j}\right)\right) .
$$

Proposition 4.1. - (See [9].) For any Lagrangian splitting $\mathfrak{d}=\mathfrak{l}_{1}+\mathfrak{l}_{2}$, the bi-vector field $\Pi_{\mathfrak{l}_{1}, \mathfrak{l}_{2}}$ on $\mathcal{L}$ is Poisson with the property that all $L_{1}$ - and $L_{2}$-orbits in $\mathcal{L}$ are Poisson submanifolds with respect to $\Pi_{\mathfrak{l}_{1}, \mathfrak{l}_{2}}$, where, for $i=1,2, L_{i}$ is the connected subgroup of $G \times G$ with Lie algebra $\mathfrak{l}_{i}$.

The rank of $\Pi_{\mathfrak{l}_{1}, \mathfrak{l}_{2}}$ can be computed as in the following Lemma 4.2. A version of Lemma 4.2 first appeared in [9], and a generalization of Lemma 4.2 can be found in [25].

LEMMA 4.2. - For $\mathfrak{l} \in \mathcal{L}$, let $\mathfrak{n}_{\mathfrak{g} \oplus \mathfrak{g}}(\mathfrak{l})$ be the normalizer subalgebra of $\mathfrak{l}$ in $\mathfrak{g} \oplus \mathfrak{g}$, and let $\left(\mathfrak{n}_{\mathfrak{g} \oplus \mathfrak{g}}(\mathfrak{l})\right)^{\perp}=\left\{x \in \mathfrak{g} \oplus \mathfrak{g}:\langle x, y\rangle=0 \forall y \in \mathfrak{n}_{\mathfrak{g} \oplus \mathfrak{g}}(\mathfrak{l})\right\}$. Set

$$
\mathcal{T}(\mathfrak{l})=\mathfrak{l}_{1} \cap \mathfrak{n}_{\mathfrak{g} \oplus \mathfrak{g}}(\mathfrak{l})+\left(\mathfrak{n}_{\mathfrak{g} \oplus \mathfrak{g}}(\mathfrak{l})\right)^{\perp} \subset \mathfrak{g} \oplus \mathfrak{g} .
$$

Then $\mathcal{T}(\mathfrak{l}) \in \mathcal{L}$, and the rank of $\Pi_{\mathfrak{l}_{1}, \mathfrak{l}_{2}}$ at $\mathfrak{l}$ is equal to $\operatorname{dim}\left(L_{1} \cdot \mathfrak{l}\right)-\operatorname{dim}\left(\mathfrak{l}_{2} \cap \mathcal{T}(\mathfrak{l})\right)$, where $L_{1} \cdot \mathfrak{l}$ is the orbit in $\mathcal{L}$ of $L_{1}$ through $\mathfrak{l}$.

Example 4.3. - It is also clear from the definition of $\Pi_{\mathfrak{l}_{1}, \mathfrak{l}_{2}}$ that $\Pi_{\mathfrak{l}_{1}, \mathfrak{l}_{2}}$ is tangent to every $(G \times G)$-orbit in $\mathcal{L}$. Thus every $(G \times G)$-orbit in $\mathcal{L}$ is a Poisson submanifold of $\left(\mathcal{L}, \Pi_{\mathfrak{l}_{1}, \mathfrak{l}_{2}}\right)$, and

$4^{\mathrm{e}}$ SÉRIE - TOME $39-2006-\mathrm{N}^{\circ} 2$ 
its closure is a Poisson subvariety. For example, let $d$ be a diagram automorphism and consider the embedding of $G$ into $\mathcal{L}$ as the $(G \times G)$-orbit through $\mathfrak{l}_{\gamma_{d}}=\left\{\left(x, \gamma_{d}(x)\right): x \in \mathfrak{g}\right\}$ :

$$
G \hookrightarrow \mathcal{L}: g \mapsto\left\{\left(x, \gamma_{d} \operatorname{Ad}_{g}(x)\right): x \in \mathfrak{g}\right\} .
$$

By Proposition 4.1, every Lagrangian splitting of $\mathfrak{g} \oplus \mathfrak{g}$ gives rise to a Poisson structure $\Pi_{\mathfrak{l}_{1}, \mathfrak{l}_{2}}$ on $G$ which extends to the closure $Z_{d}(G)$ of $G$ in $\mathcal{L}$.

Example 4.4. - By a Belavin-Drinfeld splitting we mean a Lagrangian splitting $\mathfrak{g} \oplus \mathfrak{g}=\mathfrak{l}_{1}+\mathfrak{l}_{2}$ in which $\mathfrak{l}_{1}=\mathfrak{g}_{\Delta}$. By Corollary $3.18, \mathfrak{l}_{2}$ is conjugate by an element in $G_{\Delta}$ to an $\mathfrak{l}_{S, T, d, V}$, where $(S, T, d, V)$ is a Belavin-Drinfeld system (Definition 3.17). We will also denote a BelavinDrinfeld splitting by $\mathfrak{g} \oplus \mathfrak{g}=\mathfrak{g}_{\Delta}+\mathfrak{l}_{B D}$, and denote by $\Pi_{B D}$ the corresponding Poisson structure on $\mathcal{L}$. By Proposition 4.1, all the $G_{\Delta}$-orbits in $\mathcal{L}$ as well as their closures are Poisson submanifolds with respect to any $\Pi_{B D}$. For example, for a diagram automorphism $d$, equip $G$ and $Z_{d}(G)$ with the Poisson structure $\Pi_{B D}$ via the embedding (4.2). Then every $d$-twisted conjugacy class in $G$, as well as its closure in $Z_{d}(G)$, is a Poisson subvariety with respect to every $\Pi_{B D}$. As a special case, every complex symmetric space $G / G^{\sigma}$, as well as its De Concini-Procesi compactification, inherits the Poisson structure $\Pi_{B D}$ this way. See Remark 3.22.

Lagrangian splittings of $\mathfrak{g} \oplus \mathfrak{g}$ up to conjugation by elements in $G \times G$ have been classified by P. Delorme [6]. A study of the Poisson structures $\Pi_{\mathfrak{l}_{1}, \mathfrak{l}_{2}}$ defined by arbitrary Lagrangian splittings $\mathfrak{g} \oplus \mathfrak{g}=\mathfrak{l}_{1}+\mathfrak{l}_{2}$ will be carried out in [25].

For the rest of this section, we will only be concerned with the standard Lagrangian splitting of $\mathfrak{g} \oplus \mathfrak{g}$, namely, the splitting $\mathfrak{g} \oplus \mathfrak{g}=\mathfrak{g}_{\Delta}+\mathfrak{g}_{\mathrm{st}}^{*}$, where

$$
\mathfrak{g}_{\mathrm{st}}^{*}=\mathfrak{h}_{-\Delta}+\left(\mathfrak{n} \oplus \mathfrak{n}^{-}\right) .
$$

We will denote by $\Pi_{0}$ the Poisson structure on $\mathcal{L}$ determined by the standard Lagrangian splitting. We will compute the rank of $\Pi_{0}$ everywhere on $\mathcal{L}$. As a consequence, we will see that every non-empty intersection $\mathcal{O} \cap \mathcal{O}^{\prime}$ of a $G_{\Delta}$-orbit $\mathcal{O}$ and a $\left(B \times B^{-}\right)$-orbit $\mathcal{O}^{\prime}$ is a regular Poisson subvariety of $\Pi_{0}$, and that the subgroup $H_{\Delta}=\{(h, h): h \in H\}$ of $G_{\Delta}$ acts transitively on the set of all symplectic leaves in $\mathcal{O} \cap \mathcal{O}^{\prime}$, where $H=B \cap B^{-}$.

\subsection{The rank of the Poisson structure $\Pi_{0}$}

Let $\mathcal{O}$ be a $G_{\Delta}$-orbit in $\mathcal{L}$ and $\mathcal{O}^{\prime}$ a $\left(B \times B^{-}\right)$-orbit in $\mathcal{L}$ such that $\mathcal{O} \cap \mathcal{O}^{\prime} \neq \emptyset$. Since $\left(\mathfrak{b} \oplus \mathfrak{b}^{-}\right)+\mathfrak{g}_{\Delta}=\mathfrak{g} \oplus \mathfrak{g}, \mathcal{O}$ and $\mathcal{O}^{\prime}$ intersect transversally in their $(G \times G)$-orbit. Since both $\mathcal{O}$ and $\mathcal{O}^{\prime}$ are Poisson submanifolds for $\Pi_{0}$, the intersection $\mathcal{O} \cap \mathcal{O}^{\prime}$ is a Poisson submanifold of $\left(\mathcal{L}, \Pi_{0}\right)$. Thus, it is enough to compute the rank of $\Pi_{0}$ as a Poisson structure in $\mathcal{O} \cap \mathcal{O}^{\prime}$. By Theorem 2.16, there exists a generalized Belavin-Drinfeld triple $(S, T, d)$ and $V \in \mathcal{L}_{\text {space }}\left(\mathfrak{z}_{S} \oplus \mathfrak{z}_{T}\right)$ such that $\mathcal{O}, \mathcal{O}^{\prime} \subset(G \times G) \cdot \mathfrak{l}_{S, T, d, V}$ with $\mathfrak{l}_{S, T, d, V}$ given in (2.4). By Corollaries 2.21 and 3.10, there exist $w \in W, v, v_{1} \in W^{T}$, and $m \in M_{S(v, d)}$ such that

$$
\mathcal{O}=G_{\Delta} \cdot \operatorname{Ad}_{(m, \dot{v})} \mathfrak{l}_{S, T, d, V}, \quad \mathcal{O}^{\prime}=\left(B \times B^{-}\right) \cdot \operatorname{Ad}_{\left(\dot{w}, \dot{v}_{1}\right)} \mathfrak{l}_{S, T, d, V},
$$

where $\dot{w}, \dot{v}$ and $\dot{v}_{1}$ are representatives of $w, v$, and $v_{1}$ in $G$ respectively. Set

$$
X_{S, T, d, v}=\left\{\left(z, v^{-1} z\right): z \in \mathfrak{z}_{S(v, d)}, \gamma_{d}\left(\chi_{S}(z)\right)=\chi_{T}\left(v^{-1} z\right)\right\}+V_{S} \subset \mathfrak{h} \oplus \mathfrak{h}
$$

with $V_{S}=\left\{\left(x, \gamma_{d}(x)\right): x \in \mathfrak{h}_{S}\right\}$. One shows directly that $X_{S, T, d, v}$ is a Lagrangian subspace of $\mathfrak{h} \oplus \mathfrak{h}$. 
THEOREM 4.5. - Let $\mathcal{O}$ and $\mathcal{O}^{\prime}$ be as in (4.3), and suppose that $\mathcal{O} \cap \mathcal{O}^{\prime} \neq \emptyset$. The rank of $\Pi_{0}$ at every $\mathfrak{l} \in \mathcal{O} \cap \mathcal{O}^{\prime}$ is equal to

$$
\operatorname{dim}\left(\mathcal{O} \cap \mathcal{O}^{\prime}\right)-\operatorname{dim}\left(\mathfrak{h}_{-\Delta} \cap\left(w, v_{1}\right) X_{S, T, d, v}\right),
$$

where $X_{S, T, d, v}$ is given in (4.4). In particular, $\mathcal{O} \cap \mathcal{O}^{\prime}$ is a regular Poisson submanifold of $\Pi_{0}$.

Proof. - Let $\mathfrak{l}=\operatorname{Ad}_{(g, g)} \operatorname{Ad}_{(m, \dot{v})} \mathfrak{l}_{S, T, d, V} \in \mathcal{O}$, where $g \in G$, and let $\mathcal{T}(\mathfrak{l})$ be as in Lemma 4.2. Then the rank of $\Pi_{0}$ at $\mathfrak{l}$ is equal to $\operatorname{dim}\left(G_{\Delta} \cdot \mathfrak{l}\right)-\operatorname{dim}\left(\mathfrak{g}_{\mathrm{st}}^{*} \cap \mathcal{T}(\mathfrak{l})\right)$. It follows from the definition of $\mathcal{T}(\mathfrak{l})$ that $\mathcal{T}(\mathfrak{l})=\operatorname{Ad}_{(g, g)} \mathcal{T}\left(\operatorname{Ad}_{(m, \dot{v})} \mathfrak{l}_{S, T, d, V}\right)$. Let $\mathfrak{r}_{S, T, d}^{\prime}=\left(\mathfrak{n}_{S} \oplus \mathfrak{n}_{T}^{-}\right)+\left\{\left(x, \gamma_{d}(x)\right): x \in \mathfrak{g}_{S}\right\}$, and let

$$
\mathfrak{l}_{S, T, d, v}=X_{S, T, d, v}+\mathfrak{r}_{S, T, d}^{\prime} .
$$

By Theorem 3.13,

$$
\begin{aligned}
\mathcal{T}\left(\operatorname{Ad}_{(m, \dot{v})} \mathfrak{l}_{S, T, d, V}\right) & =\mathfrak{g}_{\Delta} \cap \operatorname{Ad}_{(m, \dot{v})} \mathfrak{r}_{S, T, d}+\operatorname{Ad}_{(m, \dot{v})} \mathfrak{r}_{S, T, d}^{\prime} \\
& =\left(\mathfrak{z}_{S(v, d)}^{\prime}\right)_{\Delta}+\left(\mathfrak{g}_{S(v, d)}^{\phi}+\psi\left(\mathfrak{n}_{v}\right)\right)_{\Delta}+\operatorname{Ad}_{(m, \dot{v})} \mathfrak{r}_{S, T, d}^{\prime} .
\end{aligned}
$$

Since $\operatorname{Ad}_{(m, \dot{v})}^{-1}\left(\mathfrak{g}_{S(v, d)}^{\phi}+\psi\left(\mathfrak{n}_{v}\right)\right)_{\Delta} \subset \mathfrak{r}_{S, T, d}^{\prime}$, we have

$$
\mathcal{T}\left(\operatorname{Ad}_{(m, \dot{v})} \mathfrak{l}_{S, T, d, V}\right)=\operatorname{Ad}_{(m, \dot{v})}\left(\operatorname{Ad}_{(m, \dot{v})}^{-1}\left(\mathfrak{z}_{S(v, d)}^{\prime}\right)_{\Delta}+\mathfrak{r}_{S, T, d}^{\prime}\right)=\operatorname{Ad}_{(m, \dot{v})}\left(\mathfrak{l}_{S, T, d, v}\right) .
$$

Thus the rank of $\Pi_{0}$ at $\mathfrak{l}$ is equal to $\operatorname{Rank}_{\Pi_{0}}(\mathfrak{l})=\operatorname{dim} \mathcal{O}-\operatorname{dim}\left(\mathfrak{g}_{\mathrm{st}}^{*} \cap \operatorname{Ad}_{(g m, g \dot{v})} \mathfrak{l}_{S, T, d, v}\right)$. Let

$$
\delta=\operatorname{dim}\left(\left(\mathfrak{b} \oplus \mathfrak{b}^{-}\right) \cap \operatorname{Ad}_{(g m, g \dot{v})} \mathfrak{r}_{S, T, d}\right)-\operatorname{dim}\left(\mathfrak{g}_{\mathrm{st}}^{*} \cap \operatorname{Ad}_{(g m, g \dot{v})} \mathfrak{l}_{S, T, d, v}\right) .
$$

Then $\operatorname{Rank}_{\Pi_{0}}(\mathfrak{l})=\operatorname{dim} \mathcal{O}+\delta-\operatorname{dim}\left(\left(\mathfrak{b} \oplus \mathfrak{b}^{-}\right) \cap \operatorname{Ad}_{(g m, g \dot{v})} \mathfrak{r}_{S, T, d}\right)$. Since

$$
\operatorname{dim} \mathcal{O}^{\prime}=\operatorname{dim}\left(\mathfrak{b} \oplus \mathfrak{b}^{-}\right)-\operatorname{dim}\left(\left(\mathfrak{b} \oplus \mathfrak{b}^{-}\right) \cap \operatorname{Ad}_{(g m, g \dot{v})} \mathfrak{r}_{S, T, d}\right),
$$

we have

$$
\operatorname{Rank}_{\Pi_{0}}(\mathfrak{l})=\operatorname{dim} \mathcal{O}+\operatorname{dim} \mathcal{O}^{\prime}+\delta-\operatorname{dim}\left(\mathfrak{b} \oplus \mathfrak{b}^{-}\right)=\operatorname{dim} \mathcal{O}+\operatorname{dim} \mathcal{O}^{\prime}+\delta-2 \operatorname{dim} \mathfrak{b}
$$

Since $\mathcal{O}$ and $\mathcal{O}^{\prime}$ intersect transversally at $\mathfrak{l}$ inside the $(G \times G)$-orbit through $\mathfrak{l}$, and since $\operatorname{dim}(G \times G) \cdot \mathfrak{l}=\operatorname{dim} \mathfrak{g}-\operatorname{dim} \mathfrak{z}_{S}$ by Proposition 2.17 , we have

$$
\begin{aligned}
\operatorname{Rank}_{\Pi_{0}}(\mathfrak{l}) & =\operatorname{dim}\left(\mathcal{O} \cap \mathcal{O}^{\prime}\right)+\operatorname{dim}((G \times G) \cdot \mathfrak{l})+\delta-2 \operatorname{dim} \mathfrak{b} \\
& =\operatorname{dim}\left(\mathcal{O} \cap \mathcal{O}^{\prime}\right)-(\operatorname{dim} \mathfrak{z} S+\operatorname{dim} \mathfrak{h})+\delta .
\end{aligned}
$$

It remains to compute $\delta$. Since $\mathfrak{l} \in \mathcal{O} \cap \mathcal{O}^{\prime}$, there exist $r \in R_{S, T, d}$ and $\left(b, b^{-}\right) \in B \times B^{-}$such that $(g m, g \dot{v})=\left(b, b^{-}\right)\left(\dot{w}, \dot{v}_{1}\right) r$. Using $\operatorname{Ad}_{\left(b, b^{-}\right)}\left(\mathfrak{b} \oplus \mathfrak{b}^{-}\right)=\mathfrak{b} \oplus \mathfrak{b}^{-}$and $\operatorname{Ad}_{\left(b, b^{-}\right)} \mathfrak{g}_{\mathrm{st}^{*}}=\mathfrak{g}_{\mathrm{st}^{*}}$, we have

$$
\delta=\operatorname{dim}\left(\left(\mathfrak{b} \oplus \mathfrak{b}^{-}\right) \cap \operatorname{Ad}_{\left(\dot{w}, \dot{v}_{1}\right)} \mathfrak{r}_{S, T, d}\right)-\operatorname{dim}\left(\mathfrak{g}_{\mathrm{st}}^{*} \cap \operatorname{Ad}_{\left(\dot{w}, \dot{v}_{1}\right)} \mathfrak{l}_{S, T, d, v}\right) .
$$

Set $Y=\left(\mathfrak{n} \oplus \mathfrak{n}^{-}\right) \cap \operatorname{Ad}_{\left(\dot{w}, \dot{v}_{1}\right)}\left(\left(\mathfrak{n}_{S} \oplus \mathfrak{n}_{T}^{-}\right)+\operatorname{span}_{\mathbb{C}}\left\{\left(E_{\alpha}, \gamma_{d}\left(E_{\alpha}\right)\right): \alpha \in[S]\right\}\right)$. Then

$$
\left(\mathfrak{b} \oplus \mathfrak{b}^{-}\right) \cap \operatorname{Ad}_{\left(\dot{w}, \dot{v}_{1}\right)} \mathfrak{r}_{S, T, d}=\left(w, v_{1}\right)\left(\mathfrak{z}_{S} \oplus \mathfrak{z}_{T}+V_{S}\right)+Y .
$$

$4^{\mathrm{e}}$ SÉRIE - TOME $39-2006-\mathrm{N}^{\circ} 2$ 
Since $Y \subset \mathfrak{g}_{\mathrm{st}}^{*} \cap \operatorname{Ad}_{\left(\dot{w}, \dot{v}_{1}\right)} \mathfrak{l}_{S, T, d, v}$, we have $\mathfrak{g}_{\mathrm{st}}^{*} \cap \operatorname{Ad}_{\left(\dot{w}, \dot{v}_{1}\right)} \mathfrak{l}_{S, T, d, v}=Y+\mathfrak{h}_{-\Delta} \cap\left(w, v_{1}\right) X_{S, T, d, v}$. Thus

$$
\begin{aligned}
\delta & =\operatorname{dim}\left(\mathfrak{z}_{S} \oplus \mathfrak{z}_{T}+V_{S}\right)-\operatorname{dim}\left(\mathfrak{h}_{-\Delta} \cap\left(w, v_{1}\right) X_{S, T, d, v}\right) \\
& =\operatorname{dim} \mathfrak{z}_{S}+\operatorname{dim} \mathfrak{h}-\operatorname{dim}\left(\mathfrak{h}_{-\Delta} \cap\left(w, v_{1}\right) X_{S, T, d, v}\right) .
\end{aligned}
$$

Thus the rank of $\Pi_{0}$ at $\mathfrak{l}$ is equal to $\operatorname{dim}\left(\mathcal{O} \cap \mathcal{O}^{\prime}\right)-\operatorname{dim}\left(\mathfrak{h}_{-\Delta} \cap\left(w, v_{1}\right) X_{S, T, d, v}\right)$.

Remark 4.6. - Our conclusion that $\mathcal{O} \cap \mathcal{O}^{\prime}$ is a regular Poisson manifold for $\Pi_{0}$ follows immediately from our computation of the rank of $\Pi_{0}$. It will be shown in [25] that a similar result holds for the Poisson structure $\Pi_{\mathfrak{l}_{1}, \mathfrak{l}_{2}}$ on $\mathcal{L}$ defined by any Lagrangian splitting $\mathfrak{g} \oplus \mathfrak{g}=\mathfrak{l}_{1}+\mathfrak{l}_{2}$.

COROllary 4.7. - Equip $G$ with the Poisson structure $\Pi_{0}$ via the embedding of $G$ into $\mathcal{L}$ in (4.2) for $d=1$. Let $C$ be a conjugacy class in $G$ and let $w \in W$ be such that $C \cap\left(B^{-} w B\right) \neq \emptyset$. Then the rank of $\Pi_{0}$ at every point in $C \cap\left(B^{-} w B\right)$ is

$$
\operatorname{dim} C-l(w)-\operatorname{dim}\left(\mathfrak{h}^{-w}\right)
$$

where $l(w)$ is the length of $w$, and $\mathfrak{h}^{-w}=\{x \in \mathfrak{h}: w(x)=-x\}$. In particular, $C \cap B^{-} B$ is an open dense leaf for $C$, and $\Pi_{0}$ is degenerate on the complement of $B^{-} B \cap C$ in $C$.

Proof.-By Proposition 4.1, $C$ is a Poisson submanifold of $\left(G, \Pi_{0}\right)$. By the Bruhat decomposition, $C=\bigcup_{w \in W}\left(C \cap\left(B^{-} w B\right)\right)$. Since $B^{-} B$ is open in $G$ and $C \cap B \neq \emptyset[30$, Theorem 1, p. 69], $C \cap B^{-} B$ is open and dense in $C$. The rank formula follows from Theorem 4.5, and it follows easily that $C \cap B^{-} B$ is a symplectic leaf and $\Pi_{0}$ is degenerate on $C \cap B^{-} w B$ if $w \neq 1$.

Remark 4.8. - By Corollary 4.7, any unipotent conjugacy class (and its closure in $Z_{1}(G)$ ) has an induced Poisson structure $\Pi_{0}$ with an open symplectic leaf, although the structure is not symplectic unless the orbit is a single point. Since the unipotent variety is isomorphic to the nilpotent cone in $\mathfrak{g}^{*}$, it follows that every nilpotent orbit in $\mathfrak{g}^{*}$ has an induced Poisson structure with the same properties. It would be quite interesting to compare this structure with the KirillovKostant symplectic structure.

Example 4.9. - Consider the closed $(G \times G)$-orbit through a Lagrangian subalgebra of the form $V+\left(\mathfrak{n} \oplus \mathfrak{n}^{-}\right)$, where $V$ is any Lagrangian subspace of $\mathfrak{h} \oplus \mathfrak{h}$. Such an orbit can be identified with $G / B \times G / B^{-}$, so we can regard $\Pi_{0}$ as a Poisson structure on $G / B \times G / B^{-}$. Let $\mathcal{O}$ be a $G_{\Delta}$-orbit and let $\mathcal{O}^{\prime}$ be a $\left(B \times B^{-}\right)$-orbit in $G / B \times G / B^{-}$such that $\mathcal{O} \cap \mathcal{O}^{\prime} \neq \emptyset$. By the Bruhat decomposition of $G$, there are elements $w, u, v \in W$ such that

$$
\mathcal{O}=G_{\Delta} \cdot\left(B, w B^{-}\right), \quad \mathcal{O}^{\prime}=\left(B \times B^{-}\right) \cdot\left(u B, v B^{-}\right) .
$$

The stabilizer subgroup of $G_{\Delta} \cong G$ at the point $\left(B, \dot{w} B^{-}\right) \in G / B \times G / B^{-}$is $B \cap w\left(B^{-}\right)$. Identify $\mathcal{O} \cong G /\left(B \cap w\left(B^{-}\right)\right)$, and let $p: G \rightarrow \mathcal{O} \cong G /\left(B \cap w\left(B^{-}\right)\right)$be the projection. It is then easy to see that $\mathcal{O} \cap \mathcal{O}^{\prime}=p\left(G_{w}^{u, v}\right) \subset \mathcal{O}$, where

$$
G_{w}^{u, v}=(B u B) \cap\left(B^{-} v B^{-} w^{-1}\right) .
$$

We will refer to $G_{w}^{u, v}$ as the shifted double Bruhat cell in $G$ determined by $u, v$ and $w$. Note that $B \cap w\left(B^{-}\right)$acts freely on $G_{w}^{u, v}$ by right multiplication, so

$$
\mathcal{O} \cap \mathcal{O}^{\prime} \cong G_{w}^{u, v} /\left(B \cap w\left(B^{-}\right)\right)
$$


Since $\operatorname{dim} \mathcal{O}=\operatorname{dim} \mathfrak{g}-\operatorname{dim} \mathfrak{h}-l(w)$ and $\operatorname{dim} \mathcal{O}^{\prime}=l(u)+l(v)$, we have

$$
\operatorname{dim}\left(\mathcal{O} \cap \mathcal{O}^{\prime}\right)=\operatorname{dim} \mathcal{O}+\operatorname{dim} \mathcal{O}^{\prime}-\operatorname{dim}\left(G / B \times G / B^{-}\right)=l(u)+l(v)-l(w),
$$

and $\operatorname{dim} G_{w}^{u, v}=l(u)+l(v)+\operatorname{dim} \mathfrak{h}$. By Theorem 4.5, the rank of $\Pi_{0}$ at every point of $\mathcal{O} \cap \mathcal{O}^{\prime}$ is

$$
l(u)+l(v)-l(w)-\operatorname{dim} \mathfrak{h}^{-u^{-1} v w^{-1}},
$$

where $\mathfrak{h}^{-u^{-1} v w^{-1}}=\left\{x \in \mathfrak{h}: u^{-1} v w^{-1} x=-x\right\}$. When $w=1$, we have $\mathcal{O} \cong G / H$, and $\mathcal{O} \cap \mathcal{O}^{\prime} \cong G^{u, v} / H$, where $G^{u, v}=G_{1}^{u, v}$ is the double Bruhat cell in $G$ determined by $u$ and $v$. The set $G^{u, v} / H$ is called a reduced double Bruhat cell in [34]. In [18], Kogan and Zelevinsky constructed toric charts on symplectic leaves of $\Pi_{0}$ in $\mathcal{O} \cap \mathcal{O}^{\prime}$ (for the case when $w=1$ ) by using the so-called twisted minors that are developed in [10], and they also constructed integrable systems on the symplectic leaves. It would very interesting to generalize the Kogan-Zelevinsky construction to all symplectic leaves of $\Pi_{0}$ in $G / B \times G / B^{-}$.

\subsection{The action of $H_{\Delta}$ on the set of symplectic leaves of the Poisson structure $\Pi_{0}$}

PROPOSITION 4.10. - Let $D$ be a connected complex algebraic group with connected algebraic subgroups $A$ and $C$. Suppose there exists a connected algebraic subgroup $C_{1} \subset C$ such that the multiplication morphism $A \times C_{1} \rightarrow D$ is an isomorphism to a connected open set $U$ of $D$. Let $X$ be a homogeneous space for $D$ such that the stabilizer in $D$ of a point in $X$ is connected. Then any non-empty intersection of an A-orbit in $X$ with a $C$-orbit in $X$ is smooth and connected.

Proof. - Let $A \cdot x \cap C \cdot x$ be a non-empty intersection of orbits in $X$, and note that this intersection is smooth since the hypotheses imply that the orbits intersect transversely. We show there is a fiber bundle $\pi: V \rightarrow U$, with fiber $\pi^{-1}(e) \cong A \cdot x \cap C \cdot x$ over the identity and $V$ connected, that is trivial in the Zariski topology. This implies the connectedness of the intersection, and hence the proposition. The proof is inspired by the proof of Kleiman's transversality theorem.

Let $Y=C \cdot x$ and $Z=A \cdot x$. Let $h: D \times Y \rightarrow X$ be the action map and let $i: Z \rightarrow X$ be the obvious embedding. Let $W=(D \times Y) \times{ }_{X} Z$ be the fiber product. Then $h$ is a smooth fiber bundle (see the proof of 10.8 in [13]) and the fibers $h^{-1}(x)$ are connected. For the second claim, note that $h^{-1}(x)=\{(d, c \cdot x): d c \cdot x=x\}$ and $\psi: h^{-1}(x) \rightarrow D_{x} \cdot C$ given by $\psi(d, c \cdot x)=d$ is an isomorphism. Since $D_{x}$ and $C$ are connected, the claim follows. Thus, the induced morphism from $W \rightarrow Z$ also has connected fibers. Since $Z$ is connected, it follows that $W$ is connected. Moreover, $W$ is smooth (again by the proof of 10.8 in [13]), so $W$ is irreducible.

Let $\pi: W \rightarrow D \times Y \rightarrow D$ be the composition of the induced fiber product map with projection to the first factor. Since $\pi^{-1}(U)$ is open in $W$, it is smooth and irreducible, and thus connected. Note also that $\pi^{-1}(e) \cong Y \cap Z$. It remains to show that $\pi: \pi^{-1}(U) \rightarrow U$ is a trivial fiber bundle. We define a free left $A$ action and a free right $C_{1}$ action on $W$ by the formulas

$$
\begin{aligned}
a \cdot(d, y, z) & =(a d, y, a \cdot z), \\
c \cdot(d, y, z) & =\left(d c, c^{-1} \cdot y, z\right), \\
a \in A, \quad c \in C, \quad d & \in D, \quad y \in Y, \quad z \in Z .
\end{aligned}
$$

$A$ and $C_{1}$ have the obvious free left and right multiplication actions on $U$, and $\pi: \pi^{-1}(U) \rightarrow U$ is equivariant for these actions. It follows that the morphism

$4^{e}$ SÉRIE - TOME $39-2006-\mathrm{N}^{\circ} 2$ 


$$
\begin{aligned}
\phi: A \times C_{1} \times(A \cdot x \cap C \cdot x) & \rightarrow \pi^{-1}(U), \\
(a, c, v) & \mapsto\left(a c, c^{-1} \cdot v, a \cdot v\right), \\
a \in A, \quad c \in C_{1}, \quad v \in Y & \cap Z
\end{aligned}
$$

is a bijection, and hence is an isomorphism since $\pi^{-1}(U)$ is smooth. Thus the fiber bundle is trivial.

Remark 4.11.- We thank Michel Brion for suggesting this approach. Note that the Proposition 4.10 is false as stated if we only assume that $A \cdot C$ is open in $D$. For example, let $A=G_{\Delta}$, and let $C=\left\{\left(n h, h^{-1} n^{-}\right): n \in N, h \in H, n^{-} \in N^{-}\right\}$be the connected subgroup of $D=G \times G$ corresponding to $\mathfrak{g}_{\mathrm{st}}^{*}$. Let $X=D$ and let $D$ act on $X$ by left translation. Then the intersection of the $A$-orbit and the $C$-orbit through the identity element of $D$ is $A \cap C$ which is disconnected.

Proposition 4.12. - The intersection of any $G_{\Delta}$-orbit and any $\left(B \times B^{-}\right)$-orbit in $\mathcal{L}$ is either empty or a smooth connected subvariety of $\mathcal{L}$.

Proof. - This is a consequence of Proposition 4.10. Indeed, we take $A=G_{\Delta}, C=B \times B^{-}$, $D=G \times G$, and $C_{1}=B \times N^{-}$. The fact that the stabilizer of a point in $\mathcal{L}$ is connected follows from Lemma 2.19 .

Let $H=B \cap B^{-}$and let $H_{\Delta}=\{(h, h): h \in H\}$. For every $G_{\Delta^{-}}$-orbit $\mathcal{O}$ and every $\left(B \times B^{-}\right)$orbit $\mathcal{O}^{\prime}$ such that $\mathcal{O} \cap \mathcal{O}^{\prime} \neq \emptyset, H_{\Delta}$ clearly leaves $\mathcal{O} \cap \mathcal{O}^{\prime}$ invariant. It is easy to show that the element $R \in \bigwedge^{2}(\mathfrak{g} \oplus \mathfrak{g})$ given in (4.1) is invariant under $\operatorname{Ad}_{(h, h)}$ for every $h \in H$. Thus the Poisson structure $\Pi_{0}$ on $\mathcal{L}$ is $H_{\Delta}$-invariant. In particular, for every $h \in H, \operatorname{Ad}_{(h, h)} \mathcal{E}$ is a symplectic leaf of $\Pi_{0}$ in $\mathcal{O} \cap \mathcal{O}^{\prime}$ if $\mathcal{E}$ is.

LEMMA 4.13. - Let $\mathcal{O}$ be a $G_{\Delta^{-}}$-orbit and $\mathcal{O}^{\prime}$ a $\left(B \times B^{-}\right)$-orbit in $\mathcal{L}$ such that $\mathcal{O} \cap \mathcal{O}^{\prime} \neq \emptyset$. Let $\mathcal{E}$ be any symplectic leaf of $\Pi_{0}$ in $\mathcal{O} \cap \mathcal{O}^{\prime}$. Then the map

$$
\sigma: H \times \mathcal{E} \rightarrow \mathcal{O} \cap \mathcal{O}^{\prime}:(h, \mathfrak{l}) \mapsto \operatorname{Ad}_{(h, h)} \mathfrak{l}
$$

is a submersion.

Proof. - Let $e$ be the identity element of $H$ and let $\mathfrak{l} \in \mathcal{E}$. It is enough to show that

$$
\operatorname{dim} \operatorname{ker} \sigma_{*}(e, \mathfrak{l})=\operatorname{dim} \mathfrak{h}+\operatorname{dim} \mathcal{E}_{\mathfrak{l}}-\operatorname{dim} \mathcal{O} \cap \mathcal{O}^{\prime},
$$

where $\sigma_{*}(e, \mathfrak{l}): \mathfrak{h} \times T_{\mathfrak{l}} \mathcal{E} \rightarrow T_{\mathfrak{l}}\left(\mathcal{O} \cap \mathcal{O}^{\prime}\right)$ is the differential of $\sigma$ at $(e, \mathfrak{l})$.

We may assume that $\mathcal{O}$ and $\mathcal{O}^{\prime}$ are given in (4.3), and that

$$
\mathfrak{l}=\operatorname{Ad}_{(g m, g \dot{v})} \mathfrak{l}_{S, T, d, V}=\operatorname{Ad}_{\left(b \dot{w}, b^{-} \dot{v}_{1}\right)} \mathfrak{l}_{S, T, d, V}
$$

for some $g \in G$ and $\left(b, b^{-}\right) \in B \times B^{-}$. By Theorem 4.5, it is enough to show that

$$
\operatorname{dim}\left(\operatorname{ker} \sigma_{*}(e, \mathfrak{l})\right)=\operatorname{dim} \mathfrak{h}-\operatorname{dim}\left(\mathfrak{h}_{-\Delta} \cap\left(w, v_{1}\right) X_{S, T, d, v}\right),
$$

where $X_{S, T, d, v}$ is given in (4.4). Identify the tangent space of $\mathcal{O}$ at $\mathfrak{l}$ as

$$
T_{\mathfrak{l}} \mathcal{O} \cong \mathfrak{g}_{\Delta} /\left(\mathfrak{g}_{\Delta} \cap \operatorname{Ad}_{(g m, g \dot{v})} \mathfrak{r}_{S, T, d}\right)
$$


and let $q: \mathfrak{g}_{\Delta} \rightarrow \mathfrak{g}_{\Delta} /\left(\mathfrak{g}_{\Delta} \cap \operatorname{Ad}_{(g m, g \dot{v})} \mathfrak{r}_{S, T, d}\right)$ be the projection. Let $p: \mathfrak{g} \oplus \mathfrak{g} \rightarrow \mathfrak{g}_{\Delta}$ be the projection with respect to the decomposition $\mathfrak{g} \oplus \mathfrak{g}=\mathfrak{g}_{\Delta}+\mathfrak{g}_{\mathrm{st}}^{*}$. By the computation of $\mathcal{T}(\mathfrak{l})$ in the proof of Theorem 4.5, the tangent space of $\mathcal{E}$ at $\mathfrak{l}$ is given by

$$
T_{\mathfrak{l}} \mathcal{E}=(q \circ p)\left(\operatorname{Ad}_{(g m, g \dot{v})} \mathfrak{l}_{S, T, d, v}\right),
$$

where $\mathfrak{l}_{S, T, d, v}$ is given in (4.5). For $x \in \mathfrak{h}$, let $\kappa_{x}$ be the vector field on $\mathcal{O} \cap \mathcal{O}^{\prime}$ that generates the action of $\operatorname{Ad}_{(\exp t x, \exp t x)}$. Then $\operatorname{ker} \sigma_{*}(e, \mathfrak{l}) \cong\left\{x \in \mathfrak{h}: \kappa_{x}(\mathfrak{l}) \in T_{\mathfrak{l}} \mathcal{E}\right\}$. Let $x \in \mathfrak{h}$. If $\kappa_{x}(\mathfrak{l}) \in T_{\mathfrak{l}} \mathcal{E}$, then there exists $y \in \mathfrak{g}$ and $\left(y_{1}, y_{2}\right) \in \mathfrak{g}_{\mathrm{st}}^{*}$ with $\left(y+y_{1}, y+y_{2}\right) \in \operatorname{Ad}_{(g m, g \dot{v})} \mathfrak{l}_{S, T, d, v}$ such that

$$
(x-y, x-y) \in \mathfrak{g}_{\Delta} \cap \operatorname{Ad}_{(g m, g \dot{v})} \mathfrak{r}_{S, T, d}=\mathfrak{g}_{\Delta} \cap \operatorname{Ad}_{(g m, g \dot{v})} \mathfrak{l}_{S, T, d, v} .
$$

It follows that $\left(x+y_{1}, x+y_{2}\right) \in\left(\mathfrak{b} \oplus \mathfrak{b}^{-}\right) \cap \operatorname{Ad}_{(g m, g \dot{v})} \mathfrak{l}_{S, T, d, v}$. Let $r \in R_{S, T, d}$ be such that $(g m, g \dot{v})=\left(b \dot{w}, b^{-} \dot{v}_{1}\right) r$. Then

$$
\left(\mathfrak{b} \oplus \mathfrak{b}^{-}\right) \cap \operatorname{Ad}_{(g m, g \dot{v})} \mathfrak{l}_{S, T, d, v}=\operatorname{Ad}_{\left(b, b^{-}\right)}\left(\left(\mathfrak{b} \oplus \mathfrak{b}^{-}\right) \cap \operatorname{Ad}_{\left(\dot{w}, \dot{v}_{1}\right)} \mathfrak{l}_{S, T, d, v}\right) .
$$

Thus there exists $\left(y_{1}^{\prime}, y_{2}^{\prime}\right) \in \mathfrak{g}_{\mathrm{st}}^{*}$ such that

$$
\left(x+y_{1}^{\prime}, x+y_{2}^{\prime}\right) \in\left(\mathfrak{b} \oplus \mathfrak{b}^{-}\right) \cap \operatorname{Ad}_{\left(\dot{w}, \dot{v}_{1}\right)} \mathfrak{l}_{S, T, d, v} .
$$

If $\left(y^{\prime},-y^{\prime}\right)$ is the $\mathfrak{h}_{-\Delta}$-component of $\left(y_{1}^{\prime}, y_{2}^{\prime}\right) \in \mathfrak{g}_{\mathrm{st}}^{*}$, then $\left(x+y^{\prime}, x-y^{\prime}\right) \in\left(w, v_{1}\right) X_{S, T, d, v}$. Thus $(x, x) \in p\left(\left(w, v_{1}\right) X_{S, T, d, v}\right)$, where $p$ denotes the projection $\mathfrak{h} \oplus \mathfrak{h} \rightarrow \mathfrak{h}_{\Delta}$ along $\mathfrak{h}_{-\Delta}$. Conversely, if $x \in \mathfrak{h}$ is such that $(x, x) \in p\left(\left(w, v_{1}\right) X_{S, T, d, v}\right)$, then there exists $y^{\prime} \in \mathfrak{h}$ such that

$$
\left(x+y^{\prime}, x-y^{\prime}\right) \in\left(w, v_{1}\right) X_{S, T, d, v} \subset \operatorname{Ad}_{\left(\dot{w}, \dot{v}_{1}\right)} \mathfrak{l}_{S, T, d, v},
$$

and thus $\operatorname{Ad}_{\left(b, b^{-}\right)}\left(x+y^{\prime}, x-y^{\prime}\right) \in \operatorname{Ad}_{(g m, g \dot{v})} \mathfrak{l}_{S, T, d, v}$. Since

$$
\operatorname{Ad}_{\left(b, b^{-}\right)}\left(x+y^{\prime}, x-y^{\prime}\right)=\left(x+y^{\prime}, x-y^{\prime}\right) \bmod \left(\mathfrak{n} \oplus \mathfrak{n}^{-}\right),
$$

we see that $p\left(\operatorname{Ad}_{\left(b, b^{-}\right)}\left(x+y^{\prime}, x-y^{\prime}\right)\right)=(x, x)$, so $\kappa_{x}(\mathfrak{l}) \in T_{\mathfrak{l}} \mathcal{E}$. Thus we have shown that

$$
\operatorname{ker} \sigma_{*}(e, \mathfrak{l}) \cong\left\{x \in \mathfrak{h}:(x, x) \in p\left(\left(w, v_{1}\right) X_{S, T, d, v}\right)\right\} .
$$

It follows that $\operatorname{dim}\left(\operatorname{ker} \sigma_{*}(e, \mathfrak{l})\right)=\operatorname{dim} \mathfrak{h}-\operatorname{dim}\left(\mathfrak{h}_{-\Delta} \cap\left(w, v_{1}\right) X_{S, T, d, v}\right)$. The lemma now follows from Theorem 4.5.

THEOREM 4.14. - For every $G_{\Delta}$-orbit $\mathcal{O}$ and every $\left(B \times B^{-}\right)$-orbit $\mathcal{O}^{\prime}$ such that $\mathcal{O} \cap \mathcal{O}^{\prime} \neq \emptyset$, $H_{\Delta}$ acts transitively on the set of symplectic leaves of $\Pi_{0}$ in $\mathcal{O} \cap \mathcal{O}^{\prime}$.

Proof. - For $\mathfrak{l} \in \mathcal{O} \cap \mathcal{O}^{\prime}$, let $\mathcal{E}_{\mathfrak{l}}$ be the symplectic leaf of $\Pi_{0}$ through $\mathfrak{l}$, and let

$$
\mathcal{F}_{\mathfrak{l}}=\bigcup_{h \in H} \operatorname{Ad}_{(h, h)} \mathcal{E}_{\mathfrak{l}} \subset \mathcal{O} \cap \mathcal{O}^{\prime} .
$$

Then it is easy to see that either $\mathcal{F}_{\mathfrak{l}} \cap \mathcal{F}_{\mathfrak{l}^{\prime}}=\emptyset$ or $\mathcal{F}_{\mathfrak{l}}=\mathcal{F}_{\mathfrak{l}^{\prime}}$ for any $\mathfrak{l}, \mathfrak{l}^{\prime} \in \mathcal{O} \cap \mathcal{O}^{\prime}$. It follows from Lemma 4.13 that $\mathcal{F}_{\mathfrak{l}}$ is open in $\mathcal{O} \cap \mathcal{O}^{\prime}$ for every l. Since $\mathcal{O} \cap \mathcal{O}^{\prime}$ is connected by Proposition 4.12, $\mathcal{O} \cap \mathcal{O}^{\prime}=\mathcal{F}_{\mathfrak{l}}$ for every $\mathfrak{l} \in \mathcal{O} \cap \mathcal{O}^{\prime}$. 


\section{Lagrangian subalgebras of $\mathfrak{g} \oplus \mathfrak{h}$}

Let again $\mathfrak{g}$ be a complex semi-simple Lie algebra with Killing form $《$,$\rangle . Let \mathfrak{h} \subset \mathfrak{g}$ be a Cartan subalgebra. In this section, we will consider the direct sum Lie algebra $\mathfrak{g} \oplus \mathfrak{h}$, together with the symmetric, non-degenerate, and ad-invariant bilinear form

$$
\left\langle\left(x_{1}, y_{1}\right),\left(x_{2}, y_{2}\right)\right\rangle=\left\langle\left\langle x_{1}, x_{2}\right\rangle\right\rangle-\left\langle\left\langle y_{1}, y_{2}\right\rangle\right\rangle, \quad x_{1}, x_{2} \in \mathfrak{g}, y_{1}, y_{2} \in \mathfrak{h} .
$$

We wish to describe the variety $\mathcal{L}(\mathfrak{g} \oplus \mathfrak{h})$ of Lagrangian subalgebras of $\mathfrak{g} \oplus \mathfrak{h}$ with respect to $\langle$,$\rangle .$ We can describe all such Lagrangian subalgebras by using a theorem of Delorme [6].

Definition 5.1. - (See [6].) Let $\mathfrak{m}$ be a complex reductive Lie algebra with simple factors $\mathfrak{m}_{i}, i \in I$. A complex linear involution $\sigma$ of $\mathfrak{m}$ is called an $f$-involution if $\sigma$ does not preserve any $\mathfrak{m}_{i}$.

THEOREM 5.2. - (See [6].) Let $\mathfrak{u}$ be a complex reductive Lie algebra with a symmetric, nondegenerate, and ad-invariant bilinear form $\beta$.

(1) Let $\mathfrak{p}$ be a parabolic subalgebra of $\mathfrak{u}$ with Levi decomposition $\mathfrak{p}=\mathfrak{m}+\mathfrak{n}$, and decompose $\mathfrak{m}$ into $\mathfrak{m}=\overline{\mathfrak{m}}+\mathfrak{z}$, where $\overline{\mathfrak{m}}$ is its semi-simple part and $\mathfrak{z}$ its center. Let $\sigma$ be an $f$-involution of $\overline{\mathfrak{m}}$ such that $\overline{\mathfrak{m}}^{\sigma}$ is a Lagrangian subalgebra of $\overline{\mathfrak{m}}$ with respect to the restriction of $\beta$, and let $V$ be a Lagrangian subspace of $\mathfrak{z}$ with respect to the restriction of $\beta$. Then $\mathfrak{l}(\mathfrak{p}, \sigma, V):=\overline{\mathfrak{m}}^{\sigma} \oplus V \oplus \mathfrak{n}$ is a Lagrangian subalgebra of $\mathfrak{u}$ with respect to $\beta$.

(2) Every Lagrangian subalgebra of $\mathfrak{u}$ is $\mathfrak{l}(\mathfrak{p}, \sigma, V)$ for some $\mathfrak{p}, \sigma$, and $V$ as in (1).

PROPOSITION 5.3. - Every Lagrangian subalgebra of $\mathfrak{g} \oplus \mathfrak{h}$ with respect to $\langle$,$\rangle given in (5.1)$ is of the form $\mathfrak{n}+V$, where $\mathfrak{n}$ is the nilradical of a Borel subalgebra $\mathfrak{b}$ of $\mathfrak{g}$,V is a Lagrangian subspace of $\mathfrak{h} \oplus \mathfrak{h}$, and $\mathfrak{n}+V=\left\{\left(x+y_{1}, y_{2}\right): x \in \mathfrak{n},\left(y_{1}, y_{2}\right) \in V\right\}$.

Proof. - Applying Delorme's theorem to our case of $\mathfrak{u}=\mathfrak{g} \oplus \mathfrak{h}$ and $\langle$,$\rangle as the bilinear form \beta$, every Lagrangian subalgebra of $\mathfrak{g} \oplus \mathfrak{h}$ is of the form

$$
\mathfrak{l}=\left\{\left(x+y_{1}, y_{2}\right): x \in \overline{\mathfrak{m}}^{\sigma}+\mathfrak{n},\left(y_{1}, y_{2}\right) \in V\right\}
$$

for some parabolic subalgebra $\mathfrak{p}$ of $\mathfrak{g}$ with Levi decomposition $\mathfrak{p}=\mathfrak{m}+\mathfrak{n}=\overline{\mathfrak{m}}+\mathfrak{z}+\mathfrak{n}$, an $f$ involution $\sigma$ on $\overline{\mathfrak{m}}$, and a Lagrangian subspace $V$ of $\mathfrak{z} \oplus \mathfrak{h}$. We will now show that if $\overline{\mathfrak{m}} \neq 0$ and if $\sigma$ is an $f$-involution of $\overline{\mathfrak{m}}$, then $\overline{\mathfrak{m}}^{\sigma}$ is not an isotropic subspace of $\overline{\mathfrak{m}}$ for the restriction of the Killing form $\langle\langle$,$\rangle of \mathfrak{g}$ to $\overline{\mathfrak{m}}$. It follows that $\mathfrak{p}$ must be Borel, which gives Proposition 5.3.

Assume that $\overline{\mathfrak{m}} \neq 0$. Let $\mathfrak{m}_{i}$ be a simple factor of $\overline{\mathfrak{m}}$. Then since $\mathfrak{m}_{i}$ is simple, it has a unique nondegenerate invariant form up to scalar multiplication. Hence the Killing form $\langle$,$\rangle of \mathfrak{g}$ restricts to a scalar multiple of the Killing form of $\mathfrak{m}_{i}$. Recall that the Killing form on a maximal compact subalgebra of a semi-simple Lie algebra is negative definite. It follows that the Killing form of $\mathfrak{g}$ restricts to a nonzero positive scalar multiple of the Killing form on $\mathfrak{m}_{i}$. Suppose that $\sigma$ is an involution of $\overline{\mathfrak{m}}$ mapping $\mathfrak{m}_{i}$ to $\mathfrak{m}_{j}$ with $i \neq j$. Then $\sigma$ is an isometry with respect to the Killing form of $\mathfrak{m}_{i}$ and the Killing form of $\mathfrak{m}_{j}$. Thus, there exists a nonzero positive scalar $\mu$ such that $\left\langle\langle\sigma(x), \sigma(y)\rangle=\mu\left\langle\langle x, y\rangle, \forall x, y \in \mathfrak{m}_{i}\right.\right.$. The fixed point set $\overline{\mathfrak{m}}^{\sigma}$ contains the subspace $\left\{x+\sigma(x): x \in \mathfrak{m}_{i}\right\}$. Let $x$ be a nonzero element of a maximal compact subalgebra of $\mathfrak{m}_{i}$. Then $\langle\langle x+\sigma(x), x+\sigma(x)\rangle\rangle=(1+\mu)\langle\langle x, x\rangle\rangle \neq$,0 . Thus $\overline{\mathfrak{m}}^{\sigma}$ cannot be isotropic with respect to $\langle$,$\rangle .$

Let $G$ be the adjoint group of $\mathfrak{g}$, and let $B$ be the Borel subgroup of $G$ corresponding to a Borel subalgebra $\mathfrak{b}$. 
THEOREM 5.4. - The variety $\mathcal{L}(\mathfrak{g} \oplus \mathfrak{h})$ is isomorphic to the trivial fiber bundle over $G / B$ with fibre $\mathcal{L}_{\text {space }}(\mathfrak{h} \oplus \mathfrak{h},\langle\rangle$,$) . In particular, \mathcal{L}(\mathfrak{g} \oplus \mathfrak{h})$ is smooth with two disjoint irreducible components, corresponding to the two connected components of $\mathcal{L}_{\text {space }}(\mathfrak{h} \oplus \mathfrak{h},\langle\rangle$,$) .$

Proof. - Identify $G / B$ with the variety of all Borel subalgebras of $\mathfrak{g}$. We map $\mathcal{L}(\mathfrak{g} \oplus \mathfrak{h})$ to $G / B$ by mapping a Lagrangian algebra $\mathfrak{l}=\mathfrak{n}+V$ to the unique Borel subalgebra with nilradical $\mathfrak{n}$. The fiber over $\mathfrak{n}$ may be identified with $\mathcal{L}_{\text {space }}(\mathfrak{h} \oplus \mathfrak{h},\langle\rangle$,$) . The claim about connected components$ follows from the fact the bundle is trivial.

\section{Acknowledgement}

We would like to thank Milen Yakimov and Eugene Karolinsky for pointing out errors in a preliminary version of the paper. Discussions with Milen Yakimov enabled us to improve earlier results and solve problems in more complete form. We would also like to thank Michel Brion, William Graham, and George McNinch for useful comments and the referee for suggesting many corrections and improvements. The second author is grateful to the Hong Kong University of Science and Technology for its hospitality. The first author was partially supported by (USA)NSF grant DMS-9970102 and the second author by (USA)NSF grant DMS-0105195, HKRGC grants 701603 and 703304, and the New Staff Seeding Fund at HKU.

\section{REFERENCES}

[1] Arbarello E., Cornalba M., Griffiths P., Harris J., Geometry of Algebraic Curves, vol. 1, Springer, Berlin, 1985

[2] BÉDARD R., On the Brauer liftings for modular representations, J. Algebra 93 (1985) 332-353.

[3] Belavin A., Drinfeld V., Triangular equations and simple Lie algebras, Math. Phys. Rev. 4 (1984) 93-165.

[4] CARTER R., Finite Groups of Lie Type, Conjugacy Classes and Complex Characters, John Wiley \& Sons, New York, 1993.

[5] De Concini C., Procesi C., Complete symmetric varieties, in: Invariant Theory, Montecatini, 1982, in: Lecture Notes in Math., vol. 996, Springer, Berlin, 1983, pp. 1-44.

[6] Delorme P., Classification des triples de Manin pour les algèbres de Lie réductives complexes, with an appendix by Guillaume and Macey, J. Algebra 246 (2001) 97-174.

[7] Drinfeld V.G., On Poisson homogeneous spaces of Poisson-Lie groups, Theoret. Math. Phys. 95 (2) (1993) 226-227.

[8] Evens S., LU J.-H., Poisson harmonic forms, Kostant harmonic forms, and the $S^{1}$-equivariant cohomology of $K / T, A d v$. Math. 142 (1999) 171-220.

[9] Evens S., LU J.-H., On the variety of Lagrangian subalgebras, I, Ann. École Norm. Sup. 34 (2001) 631-668.

[10] Fomin S., Zelevinsky A., Double Bruhat cells and total positivity, J. Amer. Math. Soc. 12 (2) (1999) 335-380.

[11] Fотн P., LU J.-H., On a Poisson structure on compact symmetric spaces, Comm. Math. Phys. 251 (3) (2004) 557-566.

[12] HARRIS J., Algebraic Geometry, Springer, Berlin, 1995.

[13] HARTSHORne R., Algebraic Geometry, Springer, Berlin, 1977.

[14] Humphreys J., Linear Algebraic Groups, Springer, Berlin, 1981.

[15] Karolinsky E., A Classification of Poisson Homogeneous Spaces of Complex Reductive PoissonLie Groups, Banach Center Publ., vol. 51, Polish Acad. Sci., Warsaw, 2000.

[16] Karolinsky E., Stolin A., Classical dynamical $r$-matrices, Poisson homogeneous spaces, and Lagrangian subalgebras, Lett. Math. Phys. 60 (2002) 257-274.

[17] KNAPP A., Lie Groups Beyond an Introduction, Progr. Math., vol. 140, Birkhäuser, Basel, 1996.

4e SÉRIE - TOME $39-2006-\mathrm{N}^{\circ} 2$ 
[18] Kogan M., Zelevinsky A., On symplectic leaves and integrable systems in standard complex semisimple Poisson-Lie groups, Int. Math. Res. Not. 32 (2002) 1685-1702.

[19] Korogodski L., Soibelman Y., Algebras of Functions on Quantum Groups, Part I, Math. Surveys Monographs, vol. 56, AMS, Providence, RI, 1998.

[20] Kostant B., Lie algebra cohomology and generalized Schubert cells, Ann. of Math. 77 (1) (1963) 72-144.

[21] Kostant B., Kumar S., The nil Hecke ring and cohomology of $G / P$ for a Kac-Moody group $G$, Adv. Math. 62 (3) (1986) 187-237.

[22] LU J.-H., Coordinates on Schubert cells, Kostant's harmonic forms, and the Bruhat Poisson structure on $G / B$, Trans. Groups 4 (4) (1998) 355-374.

[23] LU J.-H., Classical dynamical $r$-matrices and homogeneous Poisson structures on $G / H$ and on $K / T$, Comm. Math. Phys. 212 (2000) 337-370.

[24] Lu J.-H., Yakimov M., On a class of double cosets in reductive algebraic groups, Int. Math. Res. Not. 13 (2005) 761-797.

[25] LU J.-H., YAKIMov M., Group orbits and regular decompositions of Poisson manifolds, in preparation.

[26] Lusztig G., Parabolic character sheaves I, Moscow Math. J. 4 (2004) 153-179.

[27] Lusztig G., Parabolic character sheaves II, Moscow Math. J. 4 (2004) 869-896.

[28] Schiffmann O., On classification of dynamical $r$-matrices, Math. Res. Lett. 5 (1998) 13-31.

[29] Slodowy P., Simple Singularities and Simple Algebraic Groups, Lecture Notes in Math., vol. 815, Springer, Berlin, 1980.

[30] Steinberg R., Conjugacy Classes in Algebraic Groups, Lecture Notes in Math., vol. 366, Springer, Berlin, 1974.

[31] SPRINGER T., Intersection cohomology of $(B \times B)$-orbit closures in group compactifications, J. Algebra 258 (1) (2002) 71-111.

[32] Winter D., Algebraic group automorphisms having finite fixed point sets, Proc. Amer. Math. Soc. 18 (1967) 371-377.

[33] Yakimov M., Symplectic leaves of complex reductive Poisson Lie groups, Duke Math. J. 112 (3) (2002) 453-509.

[34] Zelevinsky A., Connected components of real double Bruhat cells, Int. Math. Res. Not. 21 (2000) 1131-1154.

\footnotetext{
Sam Evens

Department of Mathematics,

University of Notre Dame,

Notre Dame, IN 46556, USA

E-mail: evens.1@nd.edu

Jiang-Hua LU

Department of Mathematics,

University of Hong Kong,

Pokfulam Road,

Hong Kong

E-mail: jhlu@maths.hku.hk
} 